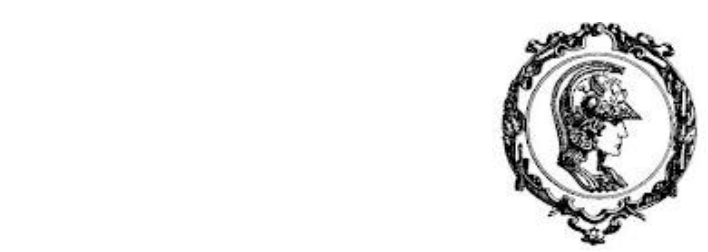

ESCOLA POLITÉCNICA DA UNIVERSIDADE DE SÃO PAULO

Ricardo Tadeu Abrahão

STUDY ON THE DISPERSION OF TITANIUM DIOXIDE PIGMENT PARTICLES IN WATER

São Paulo

2013 


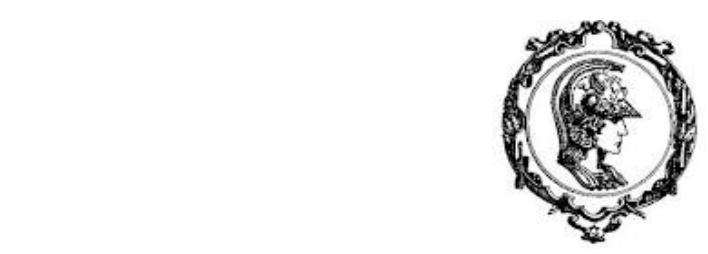

ESCOLA POLITÉCNICA DA UNIVERSIDADE DE SÃO PAULO

Ricardo Tadeu Abrahão

\section{STUDY ON THE DISPERSION OF TITANIUM DIOXIDE PIGMENT PARTICLES IN WATER}

Tese apresentada à Escola Politécnica da Universidade de São Paulo para obtenção do título de Doutor em Engenharia

São Paulo 


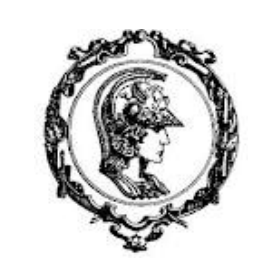

ESCOLA POLITÉCNICA DA UNIVERSIDADE DE SÃO PAULO

Ricardo Tadeu Abrahão

\section{STUDY ON THE DISPERSION OF TITANIUM DIOXIDE PIGMENT PARTICLES IN WATER}

Tese apresentada à Escola Politécnica da Universidade de São Paulo para obtenção do título de Doutor em Engenharia

Área de Concentração:

Engenharia Química

Orientadores:

Prof. Dr. Roberto Guardani

Prof. Dr. José Luis de Paiva

São Paulo 
Este exemplar foi revisado e alterado em relação à versão original, sob responsabilidade única do autor e com anuência de seu orientador.

São Paulo, 08 de Fevereiro de 2013

Ricardo Tadeu Abrahão

Prof. Dr. Roberto Guardani

Abrahão, Ricardo Tadeu

Study on the dispersion of titanium dioxide pigment particles in water / R.T. Abrahão. -- São Paulo, 2013.

$112 \mathrm{p}$.

Tese (Doutorado) - Escola Politécnica da Universidade de São Paulo. Departamento de Engenharia Química.

1.Dióxido de titânio 2.Molhabilidade 3.Umectação 4.Energia de dispersão I.Universidade de São Paulo. Escola Politécnica. Departamento de Engenharia Química II.t. 
To the women of my life with love: my wife, my mother and my sister.

And to Amelie and Julie for the company during this work, from the earliest to the latest hour. 


\section{Acknowledgments}

I would like to acknowledge and thank Prof. Dr. Roberto Guardani and Prof. Dr. José Luis de Paiva for their advice and constant support during the whole period of this work.

I would like to thank Prof. Dr. Denise Freitas Siqueira Petri for lending me the required equipment to conclude the study.

I would like to acknowledge the intern student Victor Postal for his support in doing the analysis and helping me when it was most required. Critical

I would like to acknowledge and thank everyone, especially DuPont, who helped and supported me to accomplish the objectives of this work.

I also would like to thank FUSP and the Chemistry Department of the Polytechnic School for their support.

Last but not least, I am grateful to Michael P. Diebold for supporting this work by reviewing it. 


\section{Resumo}

Dióxido de titânio é o pigmento branco mais importante de diversas indústrias incluindo as de tintas, plásticos e papel. Para atingir sua máxima eficiência no espalhamento de luz, prover a opacidade requerida ao meio em que se encontra presente, as partículas devem estar completamente dispersas neste meio.

A dispersão no meio sólido, seco ou curado dependerá da eficiência de dispersão no estado úmido, a eficiência da dispersão no estado úmido dependerá de quão efetivo foi o processo de desaglomeração. O objetivo deste trabalho é compreender como as propriedades do líquido e do sólido impactam a energia requerida para desaglomerar às partículas de dióxido de titânio pigmentário em um meio líquido.

As teorias de umectação de partículas, dispersão e estabilidade foram revisadas assim como as teorias de tensão de coesão de aglomerados, rugosidade da partícula e viscosidade e tensão superficial de líquidos. O objetivo destas revisões foi determinar os fatores que influenciam o processo de desaglomeração e a energia requerida para que isso ocorra.

Apesar das propriedades do líquido serem os principais fatores que influenciam o processo de desaglomeração (viscosidade e tensão superficial do líquido), as propriedades da partícula, ainda que sempre associadas a propriedades do líquido, desempenham um papel importante no comportamento de umectação (raio da partícula, rugosidade, volume dos poros do aglomerado e área superficial específica).

Após a caracterização das propriedades das partículas, dos aglomerados e do meio, e analisar as correlações entre as propriedades e a energia necessária para atingir o máximo da dispersão um modelo preditivo foi desenvolvido para descrever a influencia da tensão superficial e da rugosidade na energia necessária para atingir o tamanho de partícula mínimo. Este modelo se aplica a diferentes partículas com superfícies similares e partículas com superfícies diferentes com tamanhos similares. 
Palavras-chave: $\mathrm{TiO}_{2}$, Dióxido de titânio, pigmento, umectação, tensão superficial, ângulo de contato em pós, desaglomeração, energia, Washburn, surfactante, trabalho de dispersão. 


\begin{abstract}
Pigmentary titanium dioxide $\left(\mathrm{TiO}_{2}\right)$ is the most important white pigment used in several industries, including those that manufacture plastic, coatings and paper. To achieve maximum efficiency in light scattering and to deliver the required opacity to the medium in which the $\mathrm{TiO}_{2}$ is present, the $\mathrm{TiO}_{2}$ particles must be fully dispersed throughout this medium. The particle dispersion in a cured, dry, or solid medium depends on the dispersion efficiency in the wet state, which depends on the effectiveness of the deagglomeration process.

Based on the existing technical knowledge, the objective of this study is to investigate fundamental aspects in the dispersion process and to understand the effect of these processes on the required energy to deagglomerate pigmentary $\mathrm{TiO}_{2}$ particles in water.

The fundamental aspects of particle wetting, dispersion and stability are reviewed as well as the theories of the tensile strength of agglomerates, particle roughness and liquid surface tension and viscosity. Although liquid surface tension and viscosity are the main factors that influence deagglomeration, some particle-related properties (particle radius, particle shape factor, agglomerate pore volume and specific surface area) play an important role in wetting behavior. The maximum mass of water adsorbed by the agglomerates is proportional to the liquid surface tension. The liquid adsorption rate is a function of the ratio between the liquid and solid surface tensions as well as the shape factor. In the present study, for any shape factor value, the lower the liquid surface energy is in relation to the solid surface tension, the larger the water adsorption rate. After characterizing the particles, the agglomerates and the liquid medium, and obtaining correlations between all properties and the energy to achieve maximum dispersion, a predictive model is proposed to describe the influence of liquid surface tension and the particle roughness on the energy required to produce liquid dispersions with minimum particle size. This model applies to different particles with similar surfaces and to particles with different surfaces but similar sizes.
\end{abstract}


Keywords: $\mathrm{TiO}_{2}$, Titanium dioxide, pigment, wetting, wettability, surface tension, contact angle, deagglomeration, energy, capillary rise, Washburn, surfactant, work of dispersion. 


\section{List of Figures}

Figure 1 - Scanning electron microscopy of $\mathrm{TiO}_{2}$ particles (De Backer, 2010) .......................18

Figure 2 - Powder dispersion into liquid medium process .......................................................23

Figure 3 - Successive states of mobile liquid in a powder agglomerate. (A) pendular, (B)

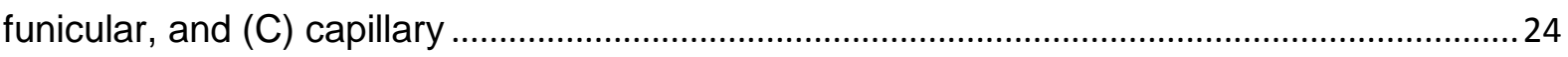

Figure 4 - Agglomerate strength due to the mobile liquid versus saturation level. (A) Pendular, (B) funicular and (C) Capillary (Pietsch; Hoffman; Rumpf, 1969).............................25

Figure 5 - Tensile strength versus water saturation level: (o) $\rho / \rho_{s}=0,35,(\square) \rho / \rho_{s}=0,30 \ldots \ldots .26$

Figure 6 - Graphical presentation of liquid bridge between 2 spherical particles (Pietsch;

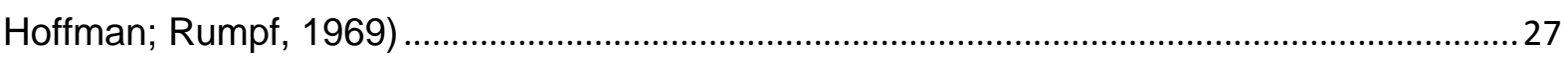

Figure 7 - Capillary flow (left) and the capillary flow equation ................................................28

Figure 8 - Cavitation process didactic presentation .................................................................. 32

Figure 9 - Potential candidates to describe cubic particles size .............................................. 36

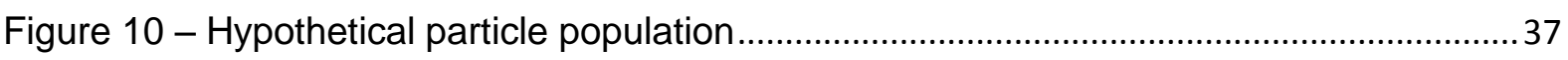

Figure 11 - Particle size distribution of Figure 10 hypothetical system....................................38

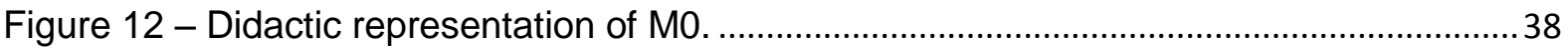

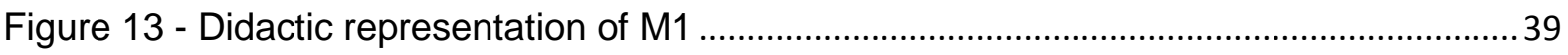

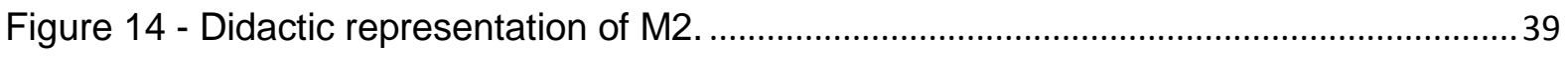

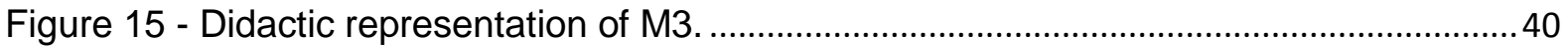

Figure 16 - A simplified scheme of a laser diffraction device for measuring particle size

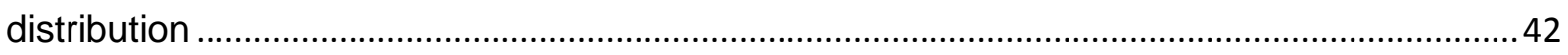

Figure 18 - Reflectance as a function of wavelength and particle size distribution. (Baneshi

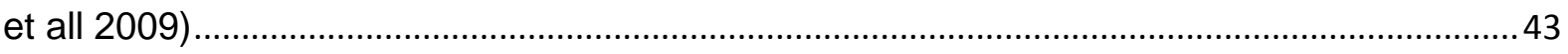

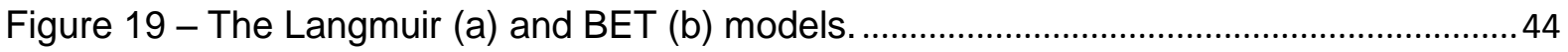
Figure 20 - Left: types of physisorption isotherms. Right: types of hysteresis loops (Sing et al., 1985) .44

Figure 21 - Typical isotherm resulting from BET analysis, in this case, for grade B sample.47 Figure 22 - Surface tension of water at $50{ }^{\circ} \mathrm{C}$ obtained for various concentrations of water mixed with a generic surfactant solution with different mole fractions (Hamid; Omar, 1999). 49 Figure 23 - Graphical summary of the properties affecting the energy required for dispersion

Figure 24 - Obscuration rate as a function of the energy applied for sample C. .56 
Figure 25 - Particle size distribution as a function of the applied energy for sample C dispersed in DI water.

Figure 26 - Output percentage and applied energy as a function of time for the ultrasound generator

Figure 27 - Mean particle sizes $D(3,2)$ and $D(4,3)$ as a function of the required energy for particle "C" dispersion.

Figure 28 - Mean particle sizes $D(3,2)$ and $D(4,3)$ as a function of the required energy for particle "C" dispersion.

Figure 29 - evolution of $\mathrm{D}(3,2)$ and $\mathrm{D}(4,3)$ as a function of particle size distribution. 60

Figure 30 - Illustration of different shape factors for particles with rougher surfaces than a spherical particle.

Figure 31 - Illustration of different shape factors for particles with smooth surfaces but of different shapes than a spherical particle

Figure 32 - Washburn methodology adaptation: influence of the tapping method during sample preparation into the repeatability and reproducibility of the methodology. 63

Figure 33 - Washburn response to sample preparation taps and number of revolutions ......63

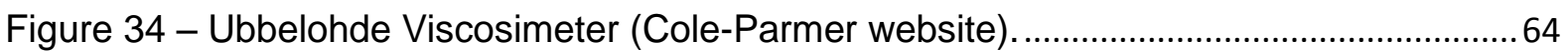

Figure 35 - Water surface tension as a function of dispersant concentration..........................66

Figure 36 - Water surface tension as a function of the concentration of dispersant.

Figure 37 - Water - surfactant solution viscosity (left axis absolute, right axis relative) as a function of the concentration of surfactant. .68

Figure 38 - Volume of liquid obtained at equilibrium point for sample A" for different values

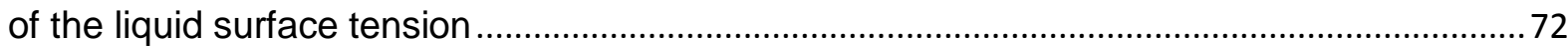

Figure 39 - Particle size distribution of samples A, A', and A". .74

Figure 40 - Relative pore volume (\%) as a function of particle class and i, for the samples tested in this study. .75

Figure 41 - Volume adsorbed by particles with different mean sizes and liquids with different values of surface tension. .78

Figure 42 - The ratio of meniscus and particle radii as a function of liquid surface tension .. 79 Figure 43 - Adsorbed liquid volume as a function of the ratio between meniscus radius and particle diameter for samples $A, A^{\prime}$, and $A^{\prime \prime}$ .79

Figure 44 - Liquid volume adsorption as a function of the ratio between meniscus and particle radii for particles $A, A^{\prime}, A^{\prime \prime}, B$, and $C$. .80

Figure 45 - Liquid volume adsorption speed as a function of the ratio between liquid and solid surface tensions for the samples used in this study. .80 
Figure 46 - Adsorption speed as a function of the ratio between liquid and solid surface tensions for different values of the shape factor $\mathrm{i}$.

Figure 47 - Fitted line plot for liquid volume adsorption speed as a function of the ratio of liquid and solid surface tension, for $\mathrm{i}=7.4$ and 8.5.

Figure 48 - Fitted line plot for liquid volume adsorption speed as a function of the liquid and solid surface tension rate $(\mathrm{i}=4.5)$.

Figure 49 - Relationship of the ratio between meniscus and particle radius and the liquid to solid surface tension ratio, for all samples.

Figure 50 - Illustration of D.I. Water surface interaction with $\mathrm{TiO}_{2}$ pellets: left: $\mathrm{TiO}_{2}$ for polyolefines with hydrophobic surface treatment; right: coatings grade with hydrophilic surface treatment.

Figure 51 - Didactic demonstration of the correlation between the ratio between meniscus and particle radii and the liquid and solid surface tensions ratio.

Figure 52 - Response surfaces regression for the wetting speed of particles $A, A^{\prime}, A^{\prime \prime}, B$, and C for shape factors (i) equal to $4.489 ; 6.615$ and 8.741

Figure 53 - Pareto chart of the standardized effects in the cubic model. .88

Figure 54 - Sensitivity analysis for the wetting speed for individual changes in the input variables, as predicted by the empirical model.

Figure 55 - Capillary pressure as a function of shape factor (i). 90

Figure 56 - Work of dispersion as a function of the relative volume of the pore occupied by the liquid penetration into the agglomerate

Figure 57 - Particle size distribution for different values of ultrasound energy applied to the samples used in this study (particles dispersed in water).

Figure 58 - Scatter plot of energy required in the dispersion for liquids with different surface tension and particles with different shape factor (i).

Figure 59 - Ultrasound energy required to achieve minimum mean particle size, $D(4,3)$, for the samples used in this study. .94

Figure 60 - Particle size distribution of samples A, A', A", B, and C. .95

Figure 61 - Required energy as a function of the liquid surface tension for all samples. ......96 Figure 62 - Required surface tension as a function of liquid surface tension and the particle shape factor $\mathrm{i}(4.5 ; 8.7$ and 7.4$)$.

Figure 63 - Exponent of the logarithmic equations in Figure 60 as a function of the particle shape factor, i.

Figure 64 - Angular coefficient as a function of the particle shape factor. .97 
Figure 65 - Comparison between observed and predicted values of required energy for

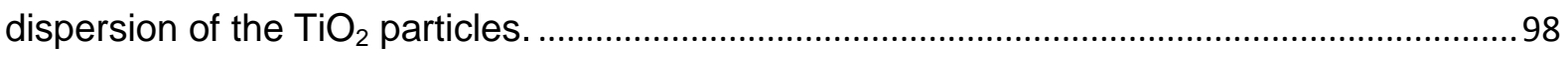
Figure 66 - Comparison between predicted values of required energy for dispersion of the $\mathrm{TiO}_{2}$ particles (log scales) .98

Figure 67 - Correlation between calculated relative agglomerates tensile strength and required energy to deagglomerate the powder. .99 Figure 68 - Scanning electron microscope image of a typical micronized $\mathrm{TiO}_{2}$ sample. 1unitary particles 2- aggregates. .(Dupont, internal technical document, authorized for this study) 100 


\section{List of Tables}

Table 1 - Comparison between the specific areas calculated based on the different

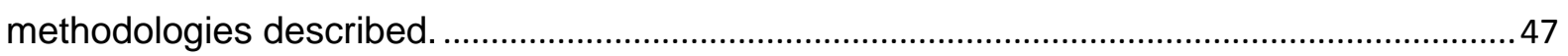

Table 2 - Surface tension of pure liquids used in this study ................................................... 48

Table 3 - Surface tension of the pure liquids and different formulations. .................................52

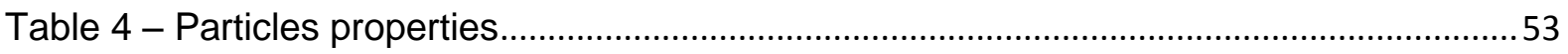

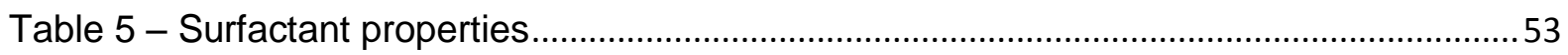

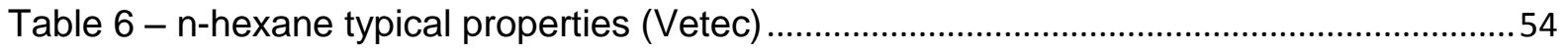

Table 7 - Typical properties of ethyl alcohol (Vetec) . ................................................................. 54

Table 8 - Typical properties of deionized water (Millipore)........................................................5

Table 9 - Sonication procedure for the samples in this study..................................................58

Table 10 - Factors to determine the design of experiments .....................................................65

Table 11 - Surface tension as a function of dispersant reproducibility analysis ......................66

Table 12 - Results of BET analysis of the samples used in this study ...................................69

Table 13 - Wetting speed results for the samples used in this study, based on the Washburn

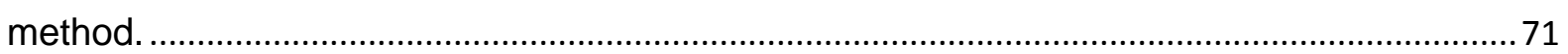

Table 14 - Calculated contact angle between particle and liquid. .......................................... 73

Table 15 - Calculated solid surface tension for the samples used in this study ...................... 73

Table 16 - Packing factor, $\mathrm{C}_{\mathrm{W}}$, for the samples used in this study .......................................... 74

Table 17 - Capillary radius, capillary pressure, and the meniscus radius of the interaction

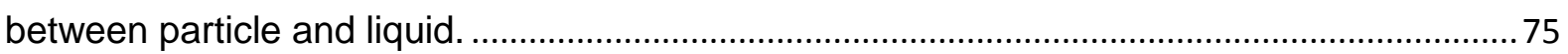

Table 18 - Dimensionless relations between capillary and particle radii and meniscus and

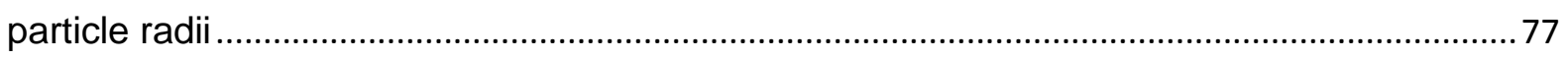

Table 19 - Coefficients of the cubic empirical model. ................................................................. 85

Table 20 - Continues Work of dispersion from the powders and liquids used in this study ..90 


\section{Contents}

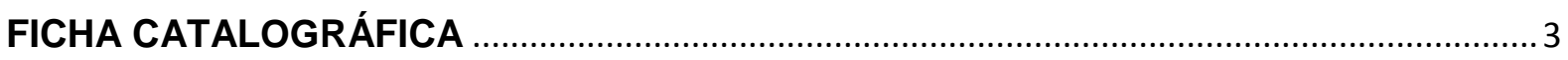

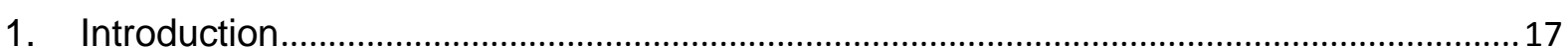

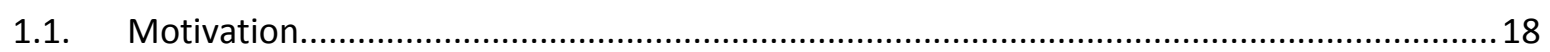

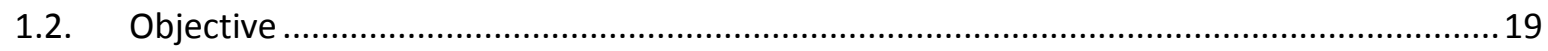

2. Fundamentals of Titanium Dioxide Particle Dispersion ....................................................2

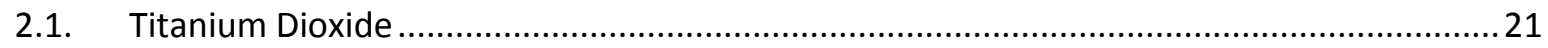

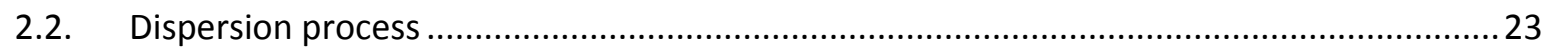

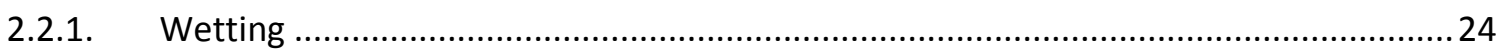

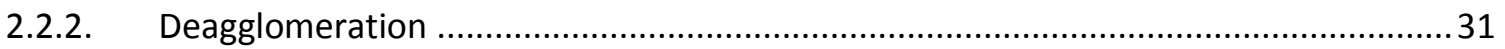

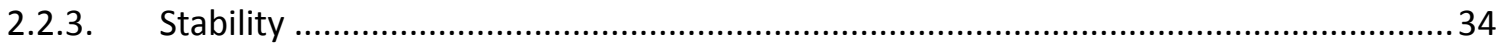

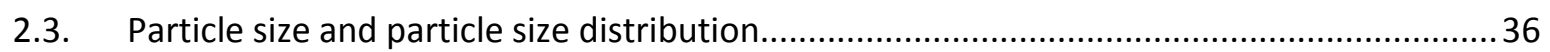

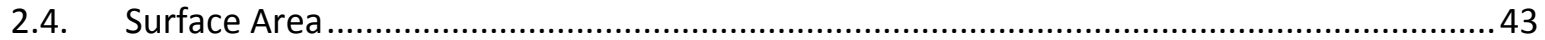

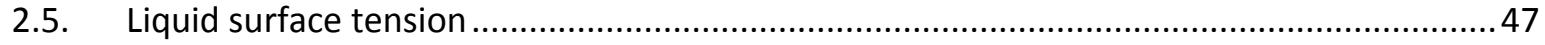

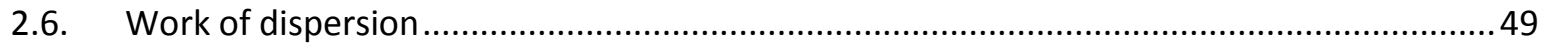

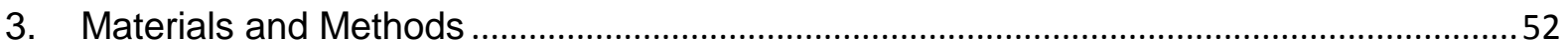

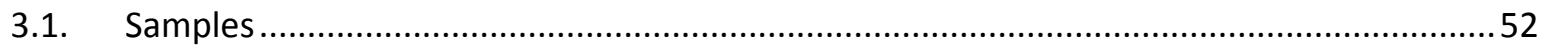

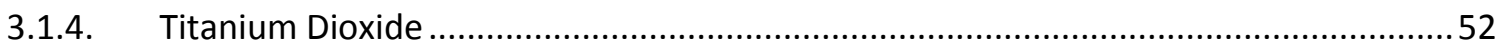

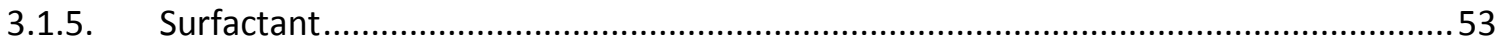

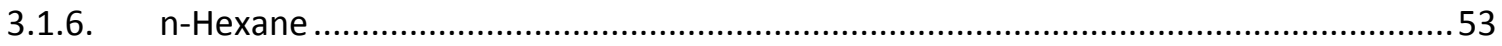

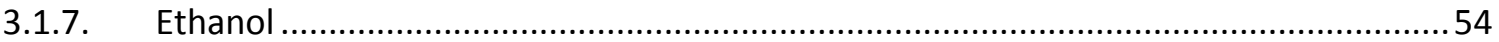

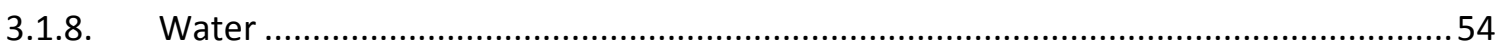

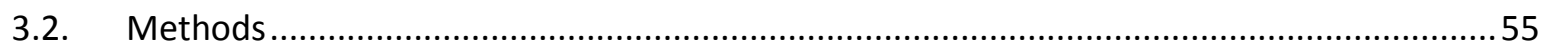

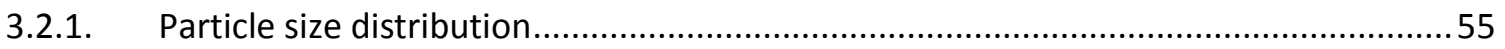

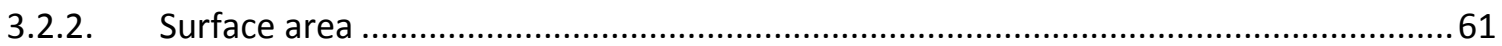

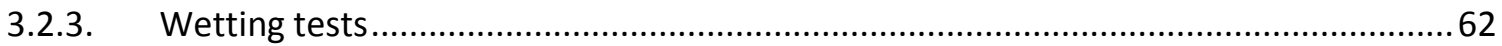

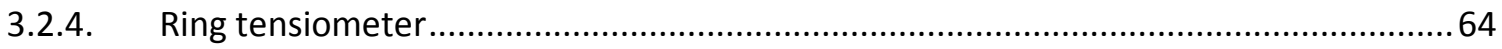

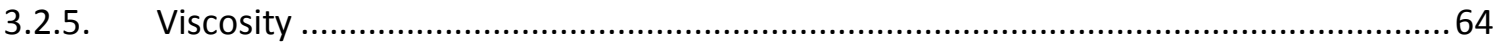




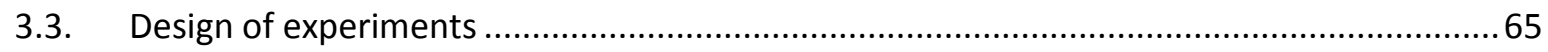

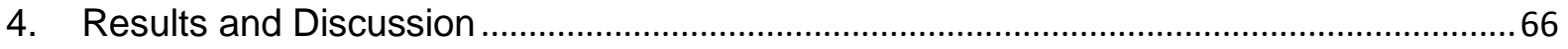

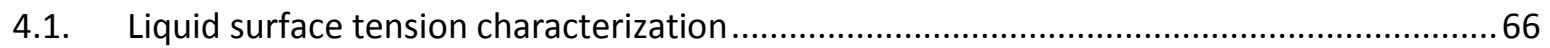

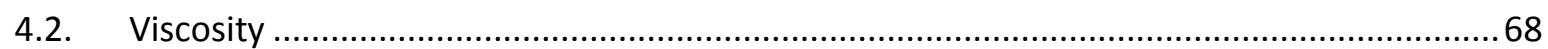

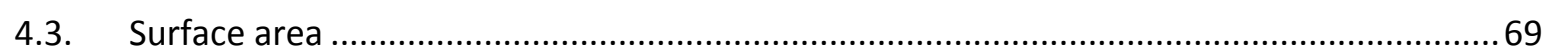

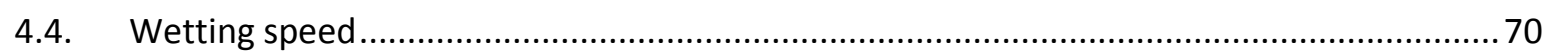

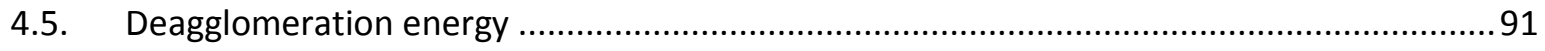

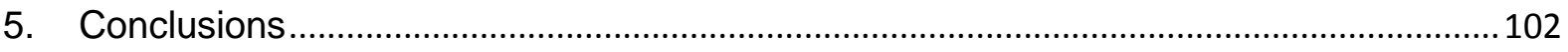

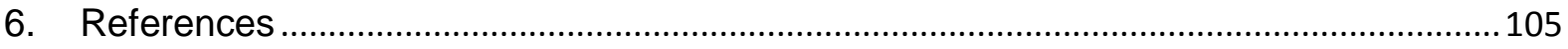




\section{Introduction}

Titanium dioxide $\left(\mathrm{TiO}_{2}\right)$ is the most important white pigment used in several industries because of its ability to scatter approximately $95 \%$ of the wavelengths of sunlight; the other $5 \%$ is the absorption of ultraviolet light (Ahmed, 1979; Auer et al., 2009; Farrokhpay, 2009; Murphy, 2001).

This study focusses on pigmentary $\mathrm{TiO}_{2}$, which is formed by particles larger than $200 \mathrm{~nm}\left(\mathrm{TiO}_{2}\right.$ particles applied in sunblock are smaller than $\left.100 \mathrm{~nm}\right)$; the function of these particles in industries that manufacture coatings, paper or plastic is to scatter visible light through refraction, reflection and diffraction.

The light scattering provided by $\mathrm{TiO}_{2}$ is highly influenced by several different factors, including particle size, crystal phase, refractive index of the binder or surrounding medium and the degree of isolation from other $\mathrm{TiO}_{2}$ particles. This degree of isolation is also referred to as degree of dispersion or lack of crowding (Thiele and French, 2005; Bruehlman, Thomas and Gonick, 1961; Tunstall and Hird, 1974; Auger, Martinez, and Stout, 2009).

To achieve the best performance in light scattering, $\mathrm{TiO}_{2}$ particles must be separated in the final film formed (coatings, plastics, paper, etc.), and the distance from particle to particle has to be at least $400 \mathrm{~nm}$ (Thiele and French, 2005,). For instance, agglomerated pairs of particles exhibit a $20 \%$ decrease in the scattering parameter associated with the hiding power of a paint film relative to the single particle.

To obtain to a condition in which the particles are separated or isolated from each other, the dispersion process as well as stability of the suspension must be carried out such that when the coating film is drying or curing, the particles should be already dispersed and isolated from each other. This state is achieved during the dispersion process. In this process, particle wetting is the first step, and if this is not done efficiently, the dispersion will not be successful. 

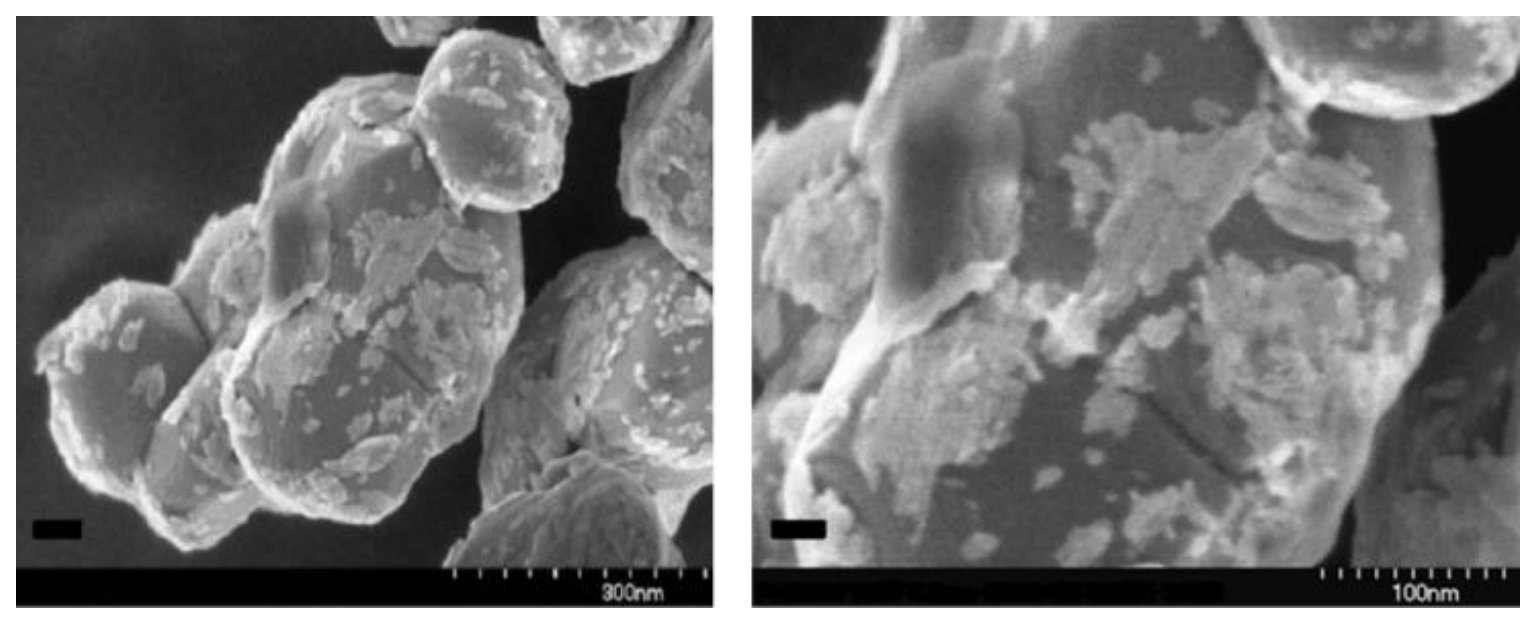

Figure 1 - Scanning electron microscopy of $\mathrm{TiO}_{2}$ particles (De Backer, 2010)

All theories regarding light scattering, wetting and other phenomena related to particle or even particle fluid interactions have been developed to describe the behavior of spherical smooth-surface particles. However, as shown in Figure 1, $\mathrm{TiO}_{2}$ particles are neither round nor smooth. Therefore, better understanding is required to predict the wetting and dispersion of non-spherical particles.

The literature includes several references to the suspension stability of slurries and solids and the forces related to it (Ayala, Hartley and Parfitt, 1986; Ding and Kawahara, 1999; Dunn and Chong, 1970; Law, Lo and Teh, 1987; Newman, Knipp and Zografi, 2012). The deagglomeration process has also been widely studied (Houivet, Fallah and Haussonne, 2002; Potente and Kretschmer, 2002; Wu and Wei, 2005; Chen et al., 1994, Zhao and Stewart, 2003).

\subsection{Motivation}

It is a common word among pigment grade $\mathrm{TiO}_{2}$ producers to promote the easiness of dispersion characteristics with no clear definition of what would mean. The coatings market seems to accept and desire this feature, but, usually, there is not a clear justification of this claim, since recent studies in this matter do not directly correlate the energy required for dispersion and the particle properties. Representative studies on this topic are listed below.

Wang et al (2006) used the Washburn method to evaluate the solid surface tension of the particle and sedimentation particle size distribution to calculate the potential energy between nanoparticles of $\mathrm{TiO}_{2}$, but no information on the required energy to disperse the particles was studied. 
Vincent et al (2011) used ultrasound to disperse nanoparticles of $\mathrm{TiO}_{2}$ and understood the effect of this application on the rheological properties of the dispersion, but no information about the dispersion state of the particles was evaluated.

Sato et al (2008) investigated the dispersion efficiency and the effect of ultrasonic irradiation on viscosity and particle size distribution in aqueous suspensions of submicrometer- and nanometer-scale $\mathrm{TiO}_{2}$ particles, and compared with the effects of ball milling, but the influence of the particle properties was left aside.

Mandzy, Grulke and Druffel (2005), applied ultrasound to investigate the particle size distribution and deagglomeration of commercially available $\mathrm{TiO}_{2}$ nanoparticles and used a zeta potential criterion to stabilize the thus formed suspension. The wettability phenomenon was not investigated and the only information from the particle surface was the zeta potential.

Therefore the motivation of this work was driven by the lack of a link among the properties of the $\mathrm{TiO}_{2}$ pigmentary particles, the dispergent liquid characteristics and the energy required to promote the full incorporation and dispersion of these particles into the dispergent.

The contribution of this study to the present state of the technique consists of the establishment of correlations between the properties of different pigment grade titanium dioxide particles and their behavior in relation to the dispersion process, which is the main step in the production of liquid pigment suspensions.

\subsection{Objective}

The objective of this study is to characterize the dispersion behavior of $\mathrm{TiO}_{2}$ pigment particles with different characteristics regarding their dispersion in a liquid medium, and to correlate this behavior with the energy required to achieve maximum dispersion. The following strategy was used to achieve the objective:

1. Characterization of liquid capillary rise to evaluate the liquid adsorption by the agglomerates

2. Characterization of the liquid surface tension as a function of surfactant concentration 
3. Characterization of the liquid capillary viscosity as a function of surfactant concentration

4. Characterization of the particle surface area and shape factor

5. Estimation of the required energy to achieve minimum particle size in the dispersion process. 


\section{Fundamentals of Titanium Dioxide Particle Dispersion}

\subsection{Titanium Dioxide}

Titanium dioxide [13463-67-7] $\left(\mathrm{TiO}_{2}\right) \quad(\mathrm{M}=79.90)$, occurs in nature in the modifications rutile, anatase, and brookite. Rutile and anatase are produced industrially in large quantities for use as pigments and catalysts and in the production of ceramic and electronic materials. Titanium dioxide is of outstanding importance as a white pigment because of its scattering properties of the visible light, which are superior to those of all other white pigments, its chemical stability, and lack of toxicity (Buxbaum, 2007).

Two different processes produce $\mathrm{TiO}_{2}$ pigments. The older sulfate process depends on the breakdown of the titanium content of the raw material ilminita, or titanium slag, with concentrated sulfuric acid at $150-220{ }^{\circ} \mathrm{C}$. Relatively pure $\mathrm{TiO}_{2}$ dehydrate is precipitated by hydrolysis of the sulfate solution, which contains colored heavy metal sulfates, sometimes in high concentration. The impurities are largely removed in further purification stages. The hydrate is then calcinated, ground and treated further.

In the chloride process, raw materials that contain titanium-Ilmenite, leucoxene, natural and synthetic rutile, titanium slag, and anatase-are chlorinated at 700$1200^{\circ} \mathrm{C}$. Titanium tetrachloride is separated from other chlorides by distillation. However, vanadium tetrachloride $\left(\mathrm{VCl}_{4}\right)$ and vanadium oxychloride $\left(\mathrm{VOCl}_{3}\right)$ must first be reduced to solid chlorides. The $\mathrm{TiCl}_{4}$ is burnt at temperatures of $900-1400{ }^{\circ} \mathrm{C}$ to form $\mathrm{TiO}_{2}$. Depending on the application, this extremely pure pigment undergoes further treatment (Clarke, 1988; Williams, 1996; Rohe, 1996; Wyckoff, 1965).

The after treatment of the pigment particles improves the weather resistance, lightfastness and dispersibility of the pigmented organic matrix. The treatment consists of coating the individual pigment particles with colorless inorganic compounds of low solubility by precipitating them onto the surface. However, this process reduces the optical performance of the pigment approximately in proportion to the decrease in the $\mathrm{TiO}_{2}$ content. The surface coatings prevent direct contact between the binder matrix and the reactive surface of the $\mathrm{TiO}_{2}$. 
The effectiveness of these coatings depends on their composition and method of application, which may result in a coating that is too porous or too dense. The treatment process also affects the dispersibility of the pigment, and therefore a compromise is often required. Usually desired are high weather resistance and good dispersibility of the pigment in the binder or matrix. These effects are controlled by using different coating densities and porosities. Other organic substances can be added during the final milling of the dried pigment. Several types of treatment are used:

1) Deposition from the gas phase occurs by hydrolysis or decomposition of volatile substances, such as chlorides or organometallic compounds. Precipitation onto the pigment surface is brought about by adding water vapor. This method is especially applicable to chloride pigments, which form under dry conditions.

2) Addition of oxides, hydroxides, or substances that can be adsorbed onto the surface during pigment grinding can produce partial coating of the pigment surface.

3) Precipitation of the coating from aqueous solutions onto the suspended $\mathrm{TiO}_{2}$ particles is achieved by batch processes in stirred tanks, which is preferred. Various compounds are deposited one after the other under optimum conditions. The patent literature on this subject is extensive. Continuous precipitation is sometimes used in mixing lines or cascades of stirred tanks. Coatings of widely differing compounds are produced in a variety of sequences. The most common are oxides, oxide hydrates, silicates, and/or phosphates of titanium, zirconium, silicon, and aluminum. For special applications, boron, tin, zinc, cerium, manganese, antimony, or vanadium compounds can be used (Buxbaum 2008).

These treatments all create a different interface between the solid particle and the medium around it, changing surface properties, such as free energy and area. Therefore, the characterization of these particles is fundamental to determine the true system of interaction that occurs when the particles are in close contact with each other and the medium. This understanding is necessary to solve problems related to the dispersion of these particles. 


\subsection{Dispersion process}

The dispersion process in water or any liquid medium is defined in three main stages (Patton, 1979):

1) Wetting refers to the displacement of gases or other contaminants that are absorbed on the surface of the pigment particles, followed by a subsequent attachment of the wetting vehicle to the pigment surface.

2) Deagglomeration refers to the separation of the particle clusters to isolated primary particles or aggregates, followed by the movement of the wetted particles into the body of the liquid vehicle to achieve permanent particle separation.

3) Stabilization is the desired state in which the de-agglomerated particles remain separated and distant from each other. This state is achieved if the particle-to-particle forces (DLVO and non-DLVO) are balanced to make this separation energetically favorable.

These stages are illustrated in Figure 2.

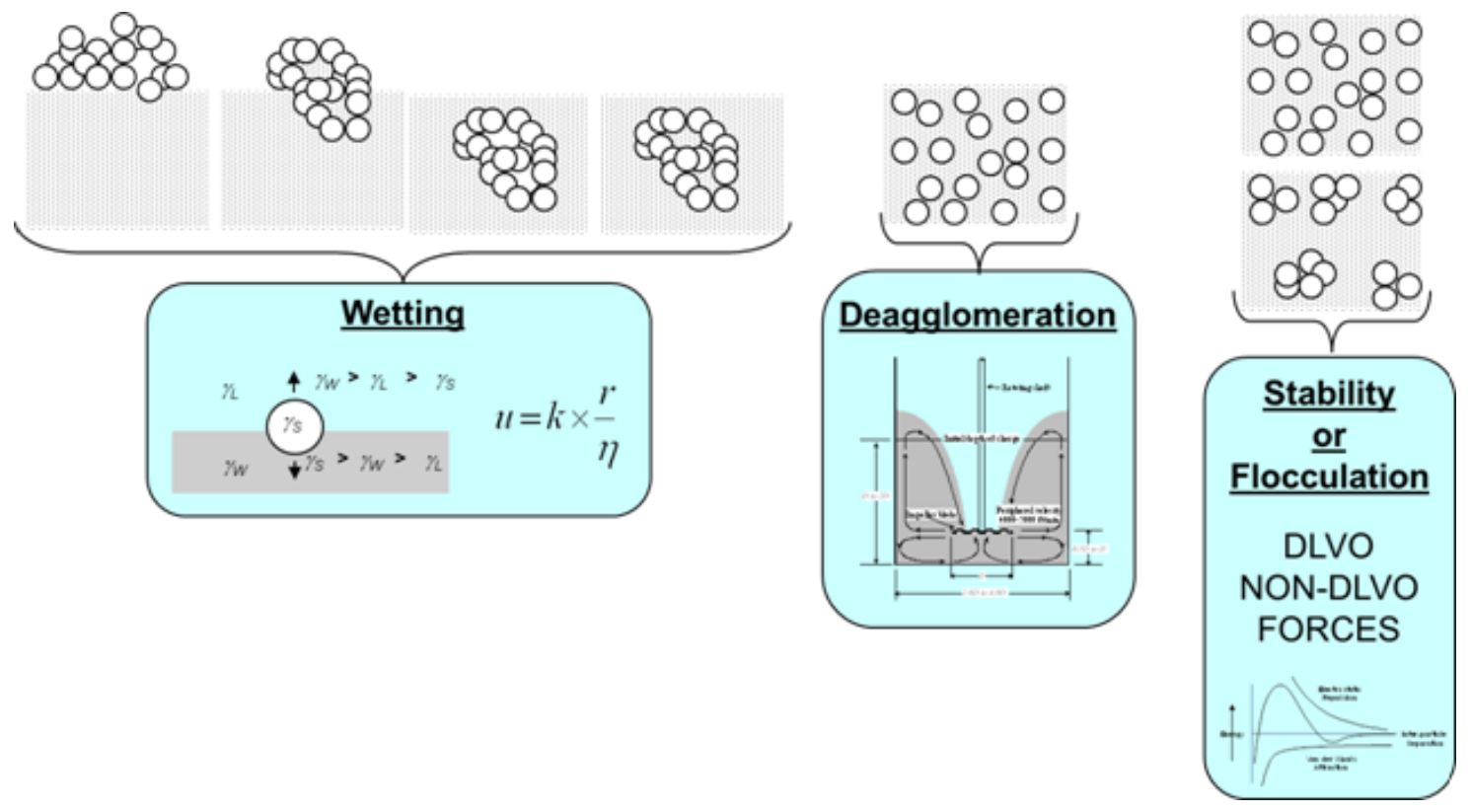

Figure 2 - Powder dispersion into liquid medium process 


\subsubsection{Wetting}

As shown in Figure 2, wetting is the first step in deagglomeration. Several studies have focused the $\mathrm{TiO}_{2}$ wetting process. Josh, Rathi and Deshpande (2009) measured the torque $(\mathrm{Ncm})$ and found that powder treated with an organic hydrophilic surface presented a lower torque during dispersion with a Cowles disperser, concluding that the pigment properties influence the wetting stage. However, in this study no information on particle size, particle size changes, or even information on particle surface was presented. It is not clear if the ease of dispersion was caused by any of these characteristics; neither was information on the agglomerates bounding energy provided.

While wetting occurs, the cohesiveness of the particles increases because of the forces involved in liquid bonding. The liquid may be present as either a mobile phase or an immobile adsorbed layer. The successive forms that a mobile liquid can take are described as pendular, funicular, and capillary states (Figure 3).

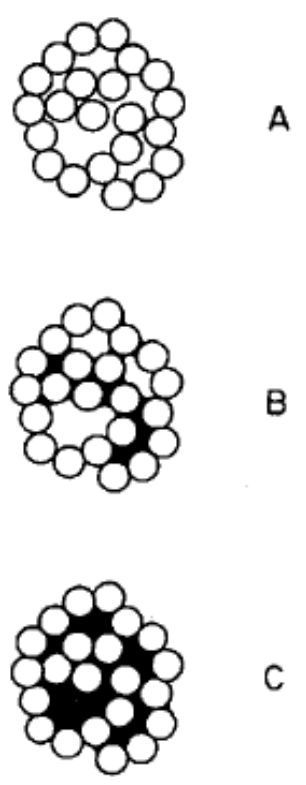

Figure 3 - Successive states of mobile liquid in
a powder agglomerate.
(A) pendular, (B)
funicular, and (C) capillary

In the pendular state, the void space in the agglomerate is partially filled with liquid that forms bridges between adjacent particles. In the funicular state, adjacent pendular rings coalesce to form a continuous liquid network interspersed with pockets of air. In this region, the strength of the agglomerate is due to both pendular 
liquid rings and capillary suction pressure. In the capillary state, liquid fills the entire void space such that there is a curvature of the liquid surface at the outer layer of the agglomerate, which results in capillary suction pressure. The maximum liquid bonding force is achieved in the capillary state.

The next stage is the slurry form. In order for the deagglomeration process to occur, the capillary state should be achieved once the energy required to break the agglomerate decreases further. The general dependence of the strength of the agglomerate on the saturation level is shown in Figure 4.

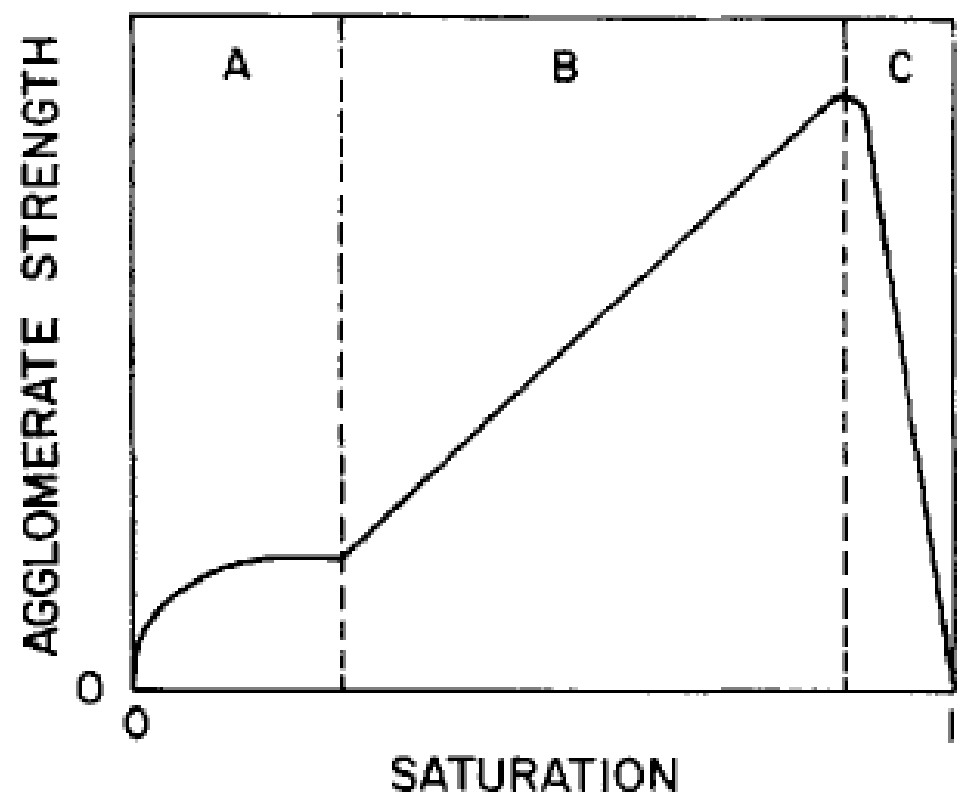

Figure 4 - Agglomerate strength due to the mobile liquid versus saturation level. (A) Pendular, (B) funicular and (C) Capillary (Pietsch; Hoffman; Rumpf, 1969)

Figure 5 presents the effect of the relation between agglomerate density $(\rho)$ and solid particle density $\left(\rho_{\mathrm{s}}\right)$. It is clear that the smaller the agglomerate density is, the larger the number of voids and the lower the number of particle-to-particle interfaces. Therefore, smaller cohesive forces are present and the maximum tensile strength of the agglomerate is less. 


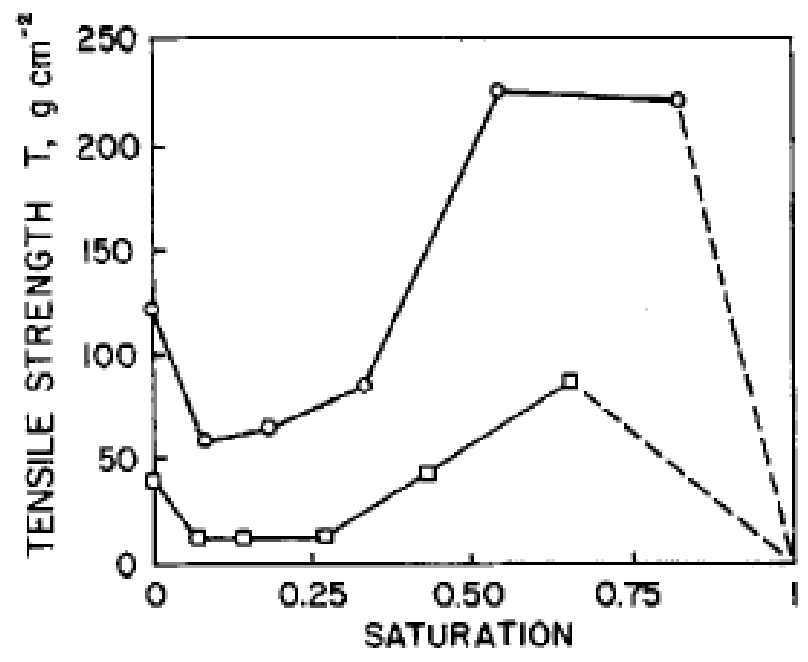

Figure 5 - Tensile strength versus water saturation level: (o) $\rho / \rho_{s}=0,35,(\square) \rho / \rho_{s}=0,30$

Saturation is given by Equation 1 where $\varepsilon$ is the porosity of the agglomerates in percentage, mo is the moisture content, $\rho_{\mathrm{s}}$ is the density of the solid and $\rho_{\mathrm{l}}$ is the liquid density (Pietsch, Hoffman and Rumpf, 1969).

$S=100 \frac{\rho_{s}}{\rho_{L}} \cdot \frac{1-\varepsilon}{\varepsilon} \cdot m o$

Equation 1

Critical saturation where the liquid rings begin contact is given by Equation 2, where $k$ is the coordination number, $\varepsilon$ is the agglomerate's porosity, $V_{p}$ is the pendular volume (interstitial volume). The latter is a function of $\beta^{2}, \delta$ and $a / x$.

$S^{\prime}=3 \cdot \frac{1-\varepsilon}{\varepsilon} \cdot \frac{k}{\pi} \cdot \frac{1}{x^{3}} \cdot v_{\mathrm{F}}\left(\beta^{\prime}, \delta, a / x\right)$

Equation 2

Refer to $\beta^{2}, \delta$ and $a / x$ in Figure 6. 


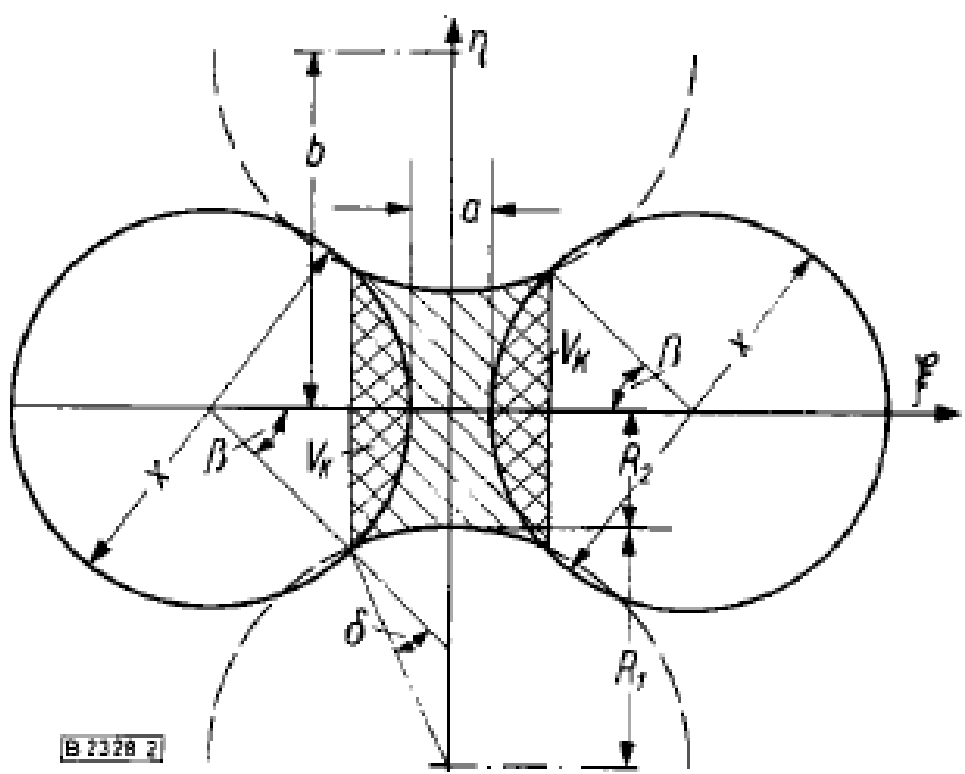

Figure 6 - Graphical presentation of liquid bridge between 2 spherical particles (Pietsch; Hoffman; Rumpf, 1969)

The tensile strength in the pendular state is then calculated based on Equation 3, where $f$ is the particle shape factor, $\varepsilon$ is agglomerate porosity, $\gamma$ is liquid surface tension, $\mathrm{x}$ is the particle unitary particle size, and $\mathrm{F}^{*}$ is the non-dimensional function for the liquid bridge force, which is dependent on the liquid bridge volume and the capillary tensile strength given by Equation 4, where $S$ is the saturation and $p$ is the capillary pressure.

$\sigma_{\mathrm{t}}=f e(1-\varepsilon) k \frac{\gamma F^{-}}{x}$

Equation 3

$\sigma_{\mathrm{t}}=S p$

Equation 4

Equation 3 presents the coordination number $(k)$, which represents the volume percentage filled by the solid. In a face-centered cubic arrangement of perfect smooth spheres, $\mathrm{k}$ is equal to $52 \%$. In a hexagonal arrangement of these particles, $\mathrm{k}$ is equal to $74 \%$, and in a random arrangement, $\mathrm{k}$ would be about $64 \%$.

Understanding how fast the saturation is achieved will allow the determination of how quickly the agglomerate reduces the internal tensile strength, thus achieving the slurring phase. The saturation speed can be defined by the capillary wetting speed. 
The penetration of liquid into particle packages is comparable to the penetration of liquid into porous material or into small tubes. Such phenomena are described as capillary flow (Constantinides and Payatakes, 2000). The flow through a cylindrical capillary tube can be described by Equation 5 , where $V$ is the capillary volume in $\mathrm{cm}^{3}, t$ is the time in seconds (s), $p$ is the capillary pressure in $\left(N / \mathrm{cm}^{2}\right), r$ is the capillary radius $(\mathrm{cm}), \eta$ is the dispergent medium viscosity and $L$ is the tube length.

$\frac{V}{i}=p \cdot\left(\frac{\pi r^{2}}{8 \pi L}\right)$

Equation 5

Since $V / t$ is equal to the product of the average liquid velocity $u$ and the tube crosssectional area $\pi r^{2}$, Equation 5 can be rearranged as Equation 6 .

$p=u \cdot\left(\frac{0 \eta L}{r^{2}}\right)$

Equation 6

The capillary pressure promotes the capillary flow throughout the agglomerates as graphically represented in Figure 7, where it is possible to see that the capillary flow does not fill the air voids inside the agglomerates, but flows along the particle surface before filling the voids.

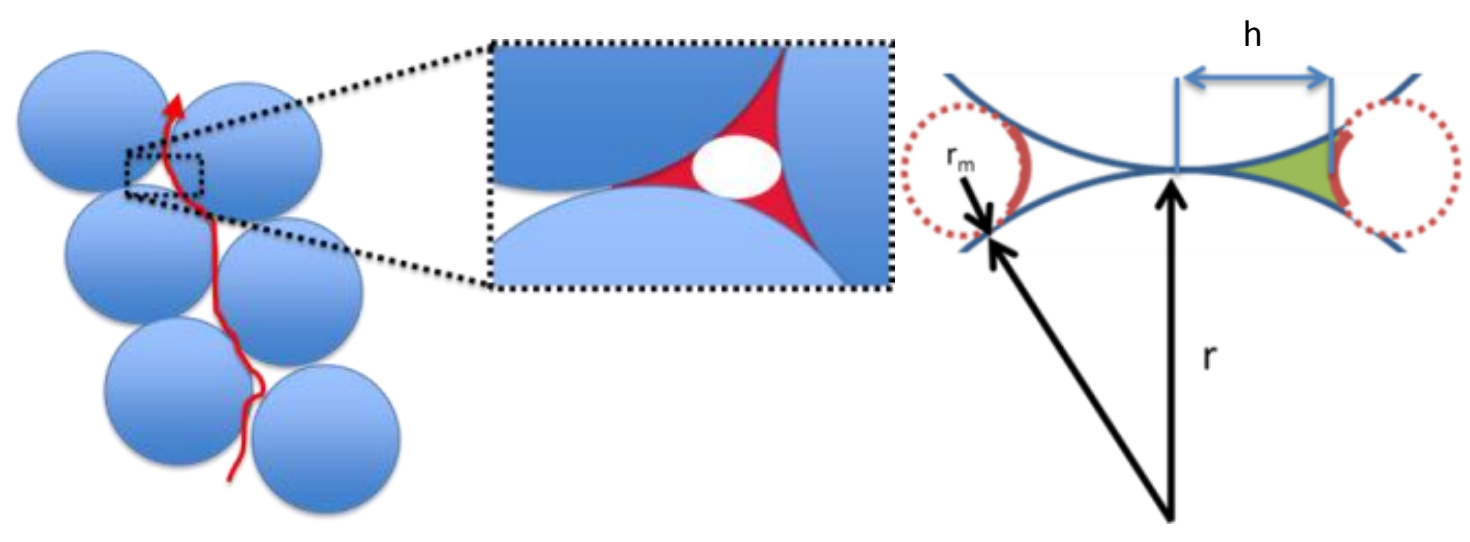

Figure 7 - Capillary flow (left) and the capillary flow equation

It is possible to find the radius of the meniscus formed by the capillary flow $\left(r_{m}\right)$ and the distance from the point of particle-to-particle contact to the center of the radius of curvature of the liquid meniscus (h). The capillary pressure $(p)$ and the liquid surface tension $(\gamma)$ in Equation 7 combine with Equation 5 to generate Equation 9. 
$p=y\left(\frac{1}{h-r_{m}}-\frac{1}{r_{\mathrm{T}}}\right)$

Equation 7

The capillary pressure can also be found by the pressure exerted by the surface tension of a curved capillary meniscus inside a capillary tube by Equation 8

$p=\frac{2 y \cos \theta}{r_{\pi 2}}$

Equation 8

Combining equations 5 and 8 :

$$
\frac{V}{t}=\frac{\gamma \cos \theta \pi\left(r_{m}\right)^{3}}{4 \eta L}
$$

Equation 9

It is important to distinguish between smooth and rough particle surfaces or spherical and non-spherical particles since this distinction can explain divergences between theory and empirical observations, and since it affects the shape factor. The relation between a rough and a smooth surface can be described as the factor $\mathrm{i}$ in Equation 9. In general, for liquid surfaces $i$ is equal to 1 and for solids $i$ is larger than 1 .

$\mathrm{i}=\frac{A_{i}}{A_{3}}$

Equation 10

where $A_{i}$ is the actual contour area of the particle surface, and $A_{e}$ is the observed projected, glossy, outside geometric or envelope area.

The faster the liquid penetrates into the agglomerate, the faster the agglomerate passes through the three saturation levels phases, achieving a smaller cohesive force point. Also interesting is that, for rougher particles the farther apart the shape factor (i) is from 1, the lesser the tensile strength. The capillary radius will then be greater, and the number of particle-particle interactions points will be reduced.

The surface tension of the penetrating liquid also plays an important role in this process because it adds to the cohesiveness of the agglomerate as well as the radius of the meniscus.

The maximum liquid absorption by the packed powder is determined by the capillary constant $\left(\mathrm{C}_{\mathrm{w}}\right)$, liquid density and viscosity $(\rho$ and $\eta)$, time (t) liquid surface tension $(\gamma)$ and the liquid-solid surface tension interactions $(\cos (\theta))$. The maximum liquid absorption is thus described by Equation 11 
$m^{2}=\left(C_{w} \rho^{2} \gamma \eta^{-1} \cos \theta\right) t$

Equation 11

Assuming the same packing factor $\left(\mathrm{C}_{\mathrm{w}}\right)$, adjusting liquid density and viscosity $(\gamma$ and $\eta)$ to each liquid in use, as $\mathrm{t} \rightarrow \infty$, the equilibrium between solid and liquid is achieved. The mass of the adsorbed liquid is proportional to the wettability of the agglomerate.

With the information above using Equation 4 and applying it to the condition where $S$ is equal to $S^{*}$ and using Equation 6 to describe the capillary pressure as a function of wetting speed the Equation 12 is formed.

$\sigma_{i \operatorname{micx}}=3 \cdot \frac{1-E}{\varepsilon} \cdot \frac{k}{\pi} \cdot \frac{1}{x^{3}} \cdot l_{x} \cdot w \cdot\left(\frac{g \eta L}{r^{2}}\right)$

Equation 12

Once $x$ is equal to $2 . r$ and simplifying Equation 12, to determine the maximum tensile strength the Equation 13 can be used.

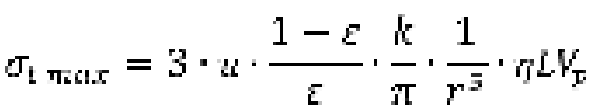

Equation 13

It is possible to observe that the maximum tensile strength is proportional to the liquid penetration velocity $(u)$ but inversely proportional to the particle radius, actually the particle radius plays the most relevant role in the agglomerate maximum tensile strength, it is taken to the fifth power meaning that small increases in particle diameter can strongly affect the agglomerate's maximum tensile strength.

To understand time's role in Equation 13, Equation 5 can be used and the time variable can be than isolated.

$\sigma_{i \operatorname{mi\alpha } x}=3 \cdot \frac{1-E}{\varepsilon} \cdot \frac{k}{\pi} \cdot \frac{1}{x^{3}} \cdot l_{x}^{g} \cdot \frac{V}{i} \cdot\left(\frac{B_{\eta} L}{\pi r^{2}}\right)$

Equation 14

For the maximum tensile strength at the pendular phase, the volume adsorbed $(\mathrm{V})$ is equal to the pendular volume $\left(V_{p}\right)$ once the liquid fulfill the voids entirely.

$\sigma_{i \operatorname{mix}}=3 \cdot \frac{1-\varepsilon}{\varepsilon} \cdot \frac{k}{\pi^{2}} \cdot \frac{1}{r^{7}} \cdot \pi L \cdot \frac{V_{5}^{2}}{!}$

Equation 15 
$i=\frac{V_{p}^{2}}{\sigma_{i \pi \omega x}} \cdot \frac{1-\varepsilon}{\underline{\varepsilon}} \cdot \frac{k}{\pi^{2}} \cdot \frac{1}{r^{7}} \cdot 3 \pi l$

Equation 16

It is clear from Equation 16 that the same factors influencing the maximum tensile strength of the agglomerates are also influencing the time to achieve its maximum value as well, and again, the particle radius is playing the most important role.

It is important to highlight that the liquid viscosity $(\eta)$ influences both, the maximum tensile strength and the time to achieve it. The higher is the liquid viscosity the higher will be the maximum tensile strength and the time to achieve it.

Another important fact to highlight is that the Equation 15 and Equation 16 relate the particle and liquid properties to a tensile strength and starts to link with a potential energy to promote the deagglomeration.

\subsubsection{Deagglomeration}

A powder is generally composed of granules and agglomerates that must be broken down to achieve dispersion. Only a mechanical effect, most commonly a grinding process, can break down the granules and agglomerates. The three main mechanisms are classically described as follows (Parfitt, 1981; Patton, 1979):

a) Particles can be crushed or sheared between two surfaces. The nature, shape, and movement of the different parts of the grinder must be taken into account under this mechanism.

b) Moving particles can be destroyed by impact against either a surface or other particles.

c) The environment surrounding particles can induce particle breakup: the viscosity of the dispersing medium must be considered, together with the effects of the electrical charges gathered on the surfaces of the particles and the wetting effect of the dispersing medium into the channels of the agglomerates, which can result in a pressure sufficiently high to break the clusters. In each case, good transmission of mechanical stress toward the 
particles requires good wetting. The energy required to de-agglomerate powders is related to the wetting efficiency (Merkus, 2009).

In coating industries, all three mechanisms happen at the same time inside the Cowlles disperser. However, this high-shear equipment is the main source of deagglomeration. When the particles are sheared, the energy for deagglomeration in liquid dispersions could come from the following sources:

1. Stirring, swirling or other weak shear forces applied to the liquid dispersion. Usually only weak bonds can be broken.

2. High-shear stirrers or dispersers. These are most often used for preparation of industrial dispersions, such as ink and paint.

3. Sonication. Three possibilities exist. In order of increasing power they are lowenergy cleaning baths (e.g., used for cleaning watches); high-energy cleaning baths (e.g., used for cleaning glassware), and high-energy tips, especially designed for difficult dispersion jobs. Note that high-power devices might cause particle breakage.

Since weak shear forces cannot overcome the cohesive forces in $\mathrm{TiO}_{2}$ agglomerates, we focus on only high-shear stirrers and high-energy sonication. The former is often found in the coatings industry, and the latter is used mainly in laboratories that specialize in particles. In both cases, cavitation is responsible for the energy applied in the agglomerate: the first results from the pressure distribution that is connected to the flow pattern; the second results from fluctuations in ultrasonic pressure. Both are affected when the pressure falls sufficiently low to form vapor bubbles.
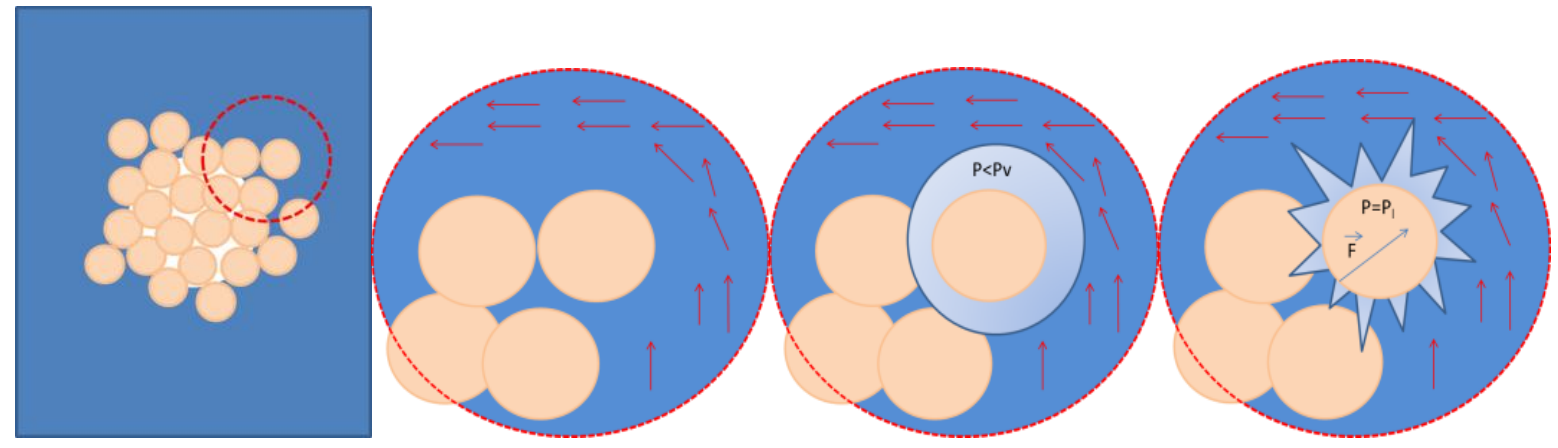

Figure 8 - Cavitation process didactic presentation 
When the bubbles containing air and vapor are created by the flow dynamics in a low-pressure region and are then transported by the flow to the higher-pressure region, they collapse if the liquid pressure is high enough. The resulting very high local pressure and velocities created in the liquid may interact with the material surface to cause erosion of elements of the equipment and the breakage of particles, including the disintegration of agglomerates. The maximum stress resulting from fluid oscillation is represented by Equation 17 (Baldyga et al., 2008).

Equation 17 was deducted by Baldyga et all in 2008 taking into consideration parameters from the particle, the agglomerates and the fluid motion and cavitation parameters. The equation also considered the bonding van der Waals forces between primary particles inside the agglomerates as well as the electrostatic repulsion forces.

$$
\tau_{0}(\vec{x}, L)=\frac{4\left|F_{0}\right|}{\pi L^{2}}
$$

Equation 17

Where $\tau_{0}$ is the maximum stress, $F_{0}$ is the particle interaction force in the agglomerates and $L$ is the agglomerate size.

\subsubsection{Mechanical}

Mechanical deagglomeration processes are traditionally used in the coatings industry as well as industries that use powder as raw material. Although several designs and configurations are based on different fundamentals, the most common equipment used is the Cowlles disperser. The disintegration of large agglomerates in these high-shear devices is dominated by an erosion mechanism and occurs in specific regions of the disperser. Hydrodynamic stresses are caused by both fluid deformation at a small scale, and inertial stress results from turbulent fluctuations, causing cavitation that occurs in regions beneath and around the impeller.

This erosion occurs in the periphery of the agglomerate. Small fragments are gradually sheared from the surface of the agglomerate. The speed of this mechanism is greatly influenced by particle wetting, liquid viscosity and radius of agglomerate pores. The latter is influenced by the unitary particle-packing factor. The factors of particle packing and shape also rule the forces bonding the particles inside the agglomerate, determining the number of contact points and the number of bonds 
connecting to other. When the liquid penetrates the pores of the agglomerate, the repulsion and attractive forces start to take place. When the erosion forces (generated by the cavitation) associated with the repulsive forces are larger than the attractive forces, deagglomeration is successful (Baldyga et al., 2008).

\subsubsection{Ultrasound}

Oscillations are generated when ultrasound waves are emitted into a fluid with suspended particles. There are three possible mechanisms for stress generation: hydrodynamic stresses generated by the flow, usually negligible in this case; hydrodynamic stress generated by the velocity difference between oscillating fluids and oscillating particles; and stress generated by cavitation events in which cavitation is generated by ultrasonic waves.

Cavitation stresses generated in the ultrasound cells are larger by many orders of magnitude than the stress generated by the oscillation of flow and fluid. A high value of cavitation stress (as high as $108 \mathrm{~Pa}$ ) supports the assumption about the rupture mechanism of deagglomeration, and the stress applied by an ultrasonic device is much larger than that applied by a disperser impeller. However, because an ultrasonic device might be easier to control, a more accurate measure of the energy applied to the suspension could be obtained (Baldyga et al., 2008).

\subsubsection{Stability}

When two colloidal particles approach one another, attractive and repulsive forces come into play. Particles can approach each other because of Brownian motion and convective transport. The main attraction and repulsion forces are the van der Waals force and the electrostatic interaction force, respectively (Hennart, Wildeboer, and van Hee, 2010). In addition to DLVO forces, steric stabilization and depletion interactions play a role in dispersion stability, but because stability is not the focus of this paper, an overview will be given without going too deeply into this subject.

The DLVO theory considers the interaction between particles by taking both attractive (Lifshitz-van der Waals) forces and repulsive (electrostatic) forces into account. When the particles approach each other too closely, attractive forces cause them to coagulate. This energetic bonding is a reversible process-changing the conditions also changes the state of the suspension. Because aggregation and 
agglomeration must be avoided, it is most important to investigate how the repulsive forces can be manipulated (Moorthaemer and Sprakel, 2006).

These repulsive forces are caused by the charge at the surface of a particle when it is in contact with the matrix solution. This net charge is denoted by a plus or minus sign (+ or - ) for all particles of equal material, which results in the repulsion of two particles that approach each other too closely.

In a solution that contains ions, this surface charge can, however, be shielded by the arrangement of oppositely charged ions around the charged surface of the particle. This is the so-called electrical double layer, which reduces the distance over which the repulsive forces are noticeable for neighboring particles. The more ions are present and the higher their valence, the more effective the electrical double layer is in shielding the surface charge.

The generic term, steric stabilization, embraces all aspects of the stabilization of colloidal particles by nonionic macromolecules. Traditionally, scientists have referred to stabilization by natural macromolecules as "protection". Presumably the electrostatic component of stability is imparted by natural macromolecules because they are usually charged (Napper, 1977).

One very characteristic feature of sterically stabilized systems is the diversity of their responses to temperature changes. Some dispersions flocculate on heating (Napper, 1970; Evans, Davison and Napper, 1972), whereas others flocculate on cooling (Napper, 1968; Evans, Davison and Napper, 1972). Other dispersions cannot, at least in principle, be flocculated at any accessible temperature (Napper, 1969). The temperature dependence of these dispersions indicates dependence on the enthalpy of the interaction among the sterically stabilized particles.

If macromolecules, such as colloids, are immersed in a solvent of smaller particles, the difference in size makes it useful to describe this mixture in terms of effective interactions by integrating the degrees of freedom of the solvent. The resulting interactions between the particles in the remaining larger component are often referred to as depletion interactions (König, Roth and Diet, 2006). The concept of effective depletion forces between two fixed big colloidal particles in a bath of small particles is generalized to a non-equilibrium situation in which the bath of small 
Brownian particles flows around the big particles at a prescribed velocity (Hamid and Omar, 1999).

\subsection{Particle size and particle size distribution.}

Because the objective of this study is to determine particle size distribution, the definition of particle size, particle size distribution, and the parameters involved is required. However, it is not easy to characterize the sizes of particles (Tavare, 1995) when their shapes are not spherical, since the size of spheres is described by their diameter. Hence, a different parameter should be used to define its size (see Figure 1). Even a cubic particle, which might be considered as regular shape, is challenging as shown in Figure 9, where three options are described: corner length, diagonal of one face, or the diagonal of the cube.

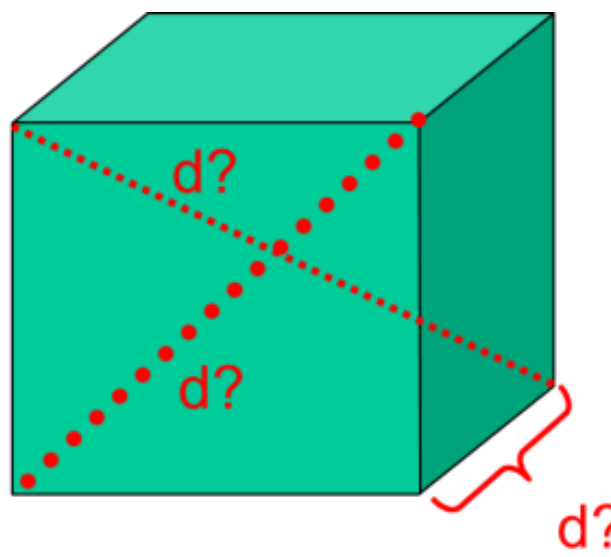

Figure 9 - Potential candidates to describe cubic particles size

Another possibility is to adopt the diameter of an equivalent sphere, which must show similar behavior when submitted to a representative test. For example, some methodologies take into consideration the speed of sedimentation and based on particle size, use the Stokes sedimentation velocity to determine the diameter of a sphere that would achieve the same velocity in that medium.

As previously mentioned, the main function of $\mathrm{TiO}_{2}$ is to scatter light and provide opacity in the medium in which it was dispersed. Therefore, the methodology for size definition used by this industry is described by the diameter of a spherical particle with the same laser or light-scattering efficiency as the analyzed particle. 
Looking for a complete particle size distribution might become confusing when a curve or even a bar plot is used as the output. A sometimes a small variation in the curvature or height of the bars is presented. Therefore, a reduction in a few numbers that represent the distribution is required. Furthermore, the moments $(M)$, which are defined in Equation 18, take into consideration the particle range and the number of particles within it.

$$
M j=\int_{0}^{\infty} n \cdot r^{j} \cdot d r
$$

Equation 18

where $r$ is the particle radius, $n$ is the population density at diameter $x$ and $j$ is the moment number of the particle size distribution. When $j$ is zero, $r^{j}$ is one; $M_{0}$ is then equal to the sum of population density in each particle size range (Abrahão, 2007). The other moments can be defined as the linear alignment of the particles $\left(M_{1}\right)$, the total surface area $\left(\mathrm{M}_{2}\right)$, or the total volume occupied by the population $\left(\mathrm{M}_{3}\right)$.

In order to better explain the meaning of moments, Abrahão (2007) developed the following explanation using the hypothetical system of Figure 10 as a particle population example.

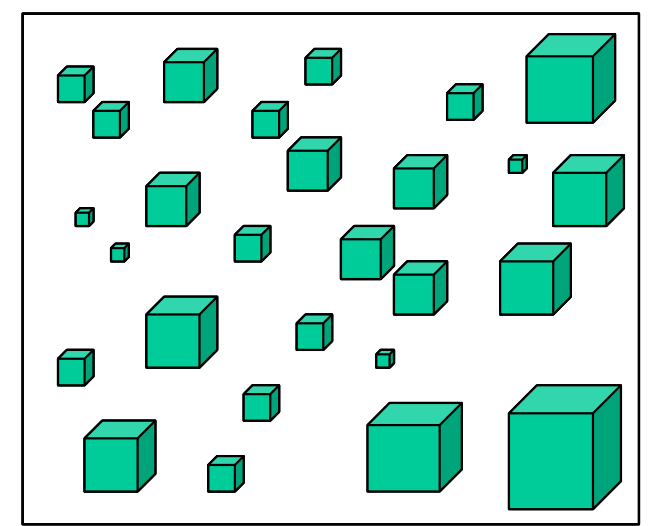

Figure 10 - Hypothetical particle population

The particle size distribution can be graphically described as in Figure 11. 


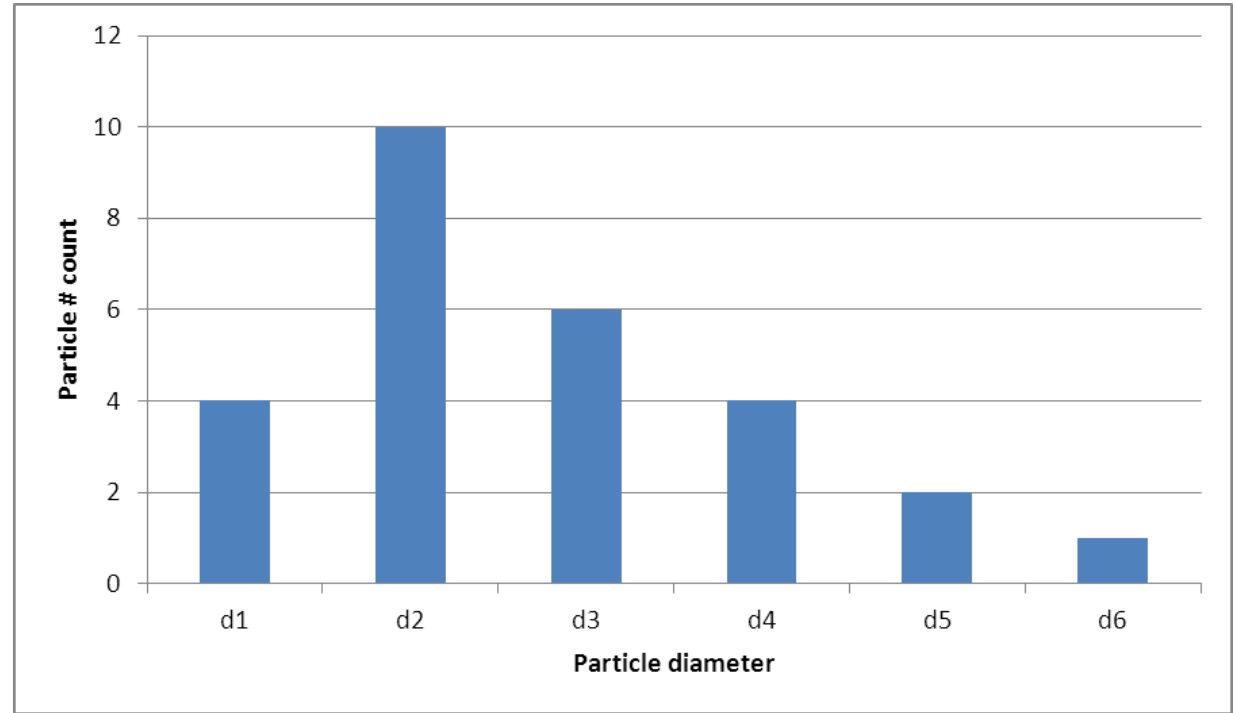

Figure 11 - Particle size distribution of Figure 10 hypothetical system.

The zero moment (M0), as defined by Equation 18 applied to the hypothetical system of Figure 10 can be described as follows:

$$
\begin{gathered}
M 0\left(r_{0}, t\right)=\int_{0}^{\infty} n \cdot r^{0} \cdot d r \\
M 0=\sum_{1}^{\infty} n \cdot r_{i}^{0} \\
M 0=4 \cdot r_{1}{ }^{0}+10 \cdot r_{2}{ }^{0}+6 \cdot r_{3}{ }^{0}+4 \cdot r_{4}{ }^{0}+2 \cdot r_{5}{ }^{0}+1 \cdot r_{6}{ }^{0}
\end{gathered}
$$

Since the diameter of the particle is taken to the zero power it is disregarded. The result is the simple number of particles per diameter size and can be didactically represented in Figure 12.

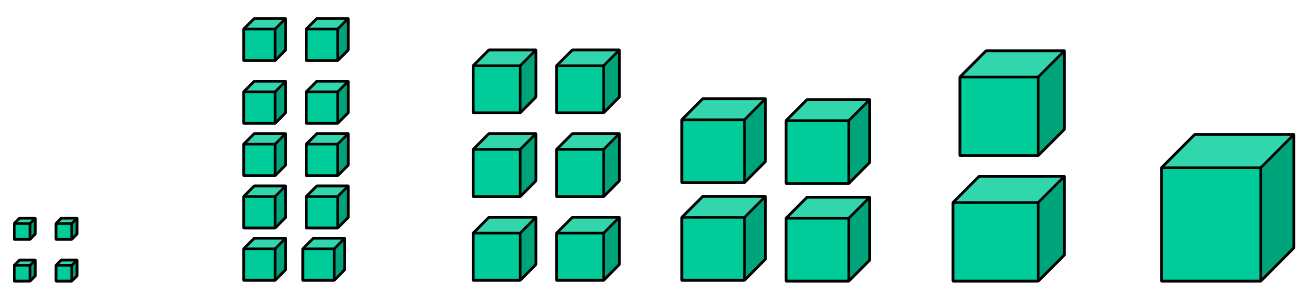

Figure 12 - Didactic representation of M0.

The first moment (order 1 moment) (M1) applied to the hypothetical system of Figure 10 can be described as follows:

$$
\begin{gathered}
M 1\left(r_{0}, t\right)=\int_{0}^{\infty} n \cdot r^{1} \cdot d r \\
M 1=\sum_{1}^{\infty} n \cdot r_{i}^{1}
\end{gathered}
$$




$$
\mathrm{M} 1=4 . r 1^{1}+10 . r 2^{1}+6 . r 3^{1}+4 . r 4^{1}+2 . r 5^{1}+1 . r 6^{1}
$$

In this case the particle diameter is taken to the first power and as a consequence, the diameter is taken into consideration and the result is a sum of the particle diameters and can be associated to a line formed by the particles when lined up and can be didactically represented in Figure 13 .

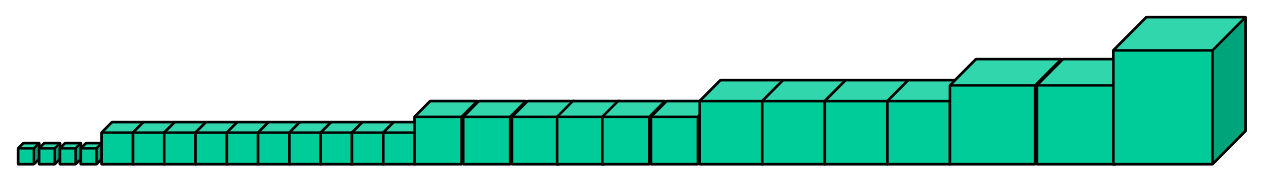

Figure 13 - Didactic representation of M1

The $2^{\text {nd }}$ order moment (M2) applied to the hypothetical system of Figure 10 can be described as follows:

$$
\begin{gathered}
M 2\left(r_{0}, t\right)=\int_{0}^{\infty} n \cdot r^{2} \cdot d r \\
M 2=\sum_{1}^{\infty} n \cdot r_{i}^{2} \\
M 2=4 \cdot r_{1}^{2}+10 \cdot r_{2}^{2}+6 \cdot r_{3}^{2}+4 \cdot r_{4}^{2}+2 \cdot r_{5}^{2}+1 \cdot r_{6}^{2}
\end{gathered}
$$

As the particle diameter is squared, M2 represents the total surface area of the particle system and can be didactically represented by Figure 14 .
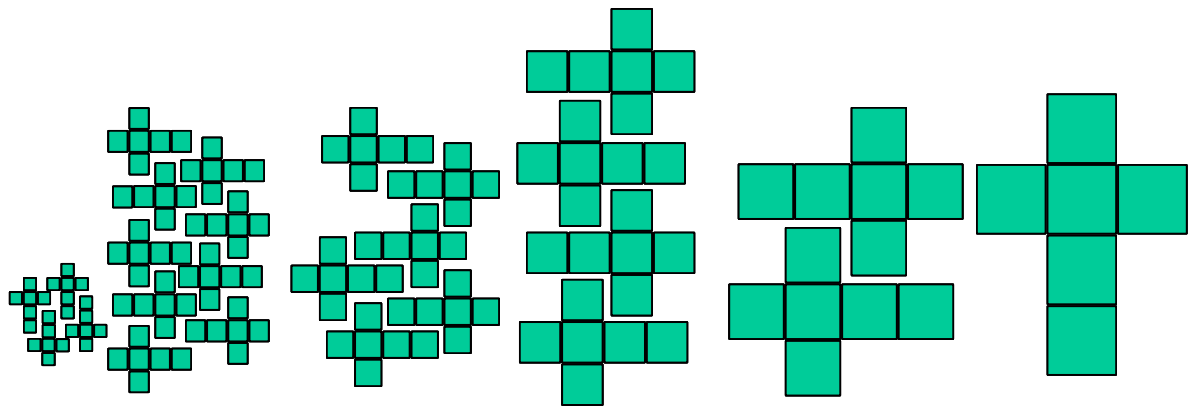

Figure 14 - Didactic representation of M2.

The $3^{\text {rd }}$ order moment (M3) applied to the hypothetical system of Figure 10 can be described as follows:

$$
\begin{gathered}
M 3\left(r_{0}, t\right)=\int_{0}^{\infty} n \cdot r^{3} \cdot d r \\
M 3=\sum_{1}^{\infty} n \cdot r_{i}^{3}
\end{gathered}
$$




$$
\mathrm{M} 3=4 \cdot r_{1}{ }^{3}+10 \cdot r_{2}{ }^{3}+6 \cdot r_{3}{ }^{3}+4 \cdot r_{4}{ }^{3}+2 \cdot r_{5}{ }^{3}+1 \cdot r_{6}{ }^{3}
$$

M3 is known as the "volumetric" moment because the particle diameter is taken to the third power and is associated to the total volume of the particles. It can be represented by Figure 15. Since M3 is related to the volume of the particles it is also associated to the total mass of the particles present in the system.

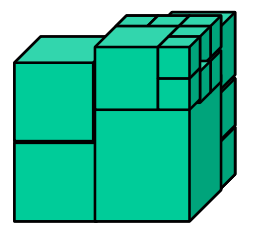

Figure 15 - Didactic representation of M3.

The moments of larger order like M4 and M5 have the particle diameter taken to the $4^{\text {th }}$ and $5^{\text {th }}$ power respectively and cannot be represented in a three dimensional perspective but are also determined by Equation 18 and described as follows:

$$
\begin{gathered}
M 4\left(r_{0}, t\right)=\int_{0}^{\infty} n \cdot r^{4} \cdot d r \quad M 5\left(r_{0}, t\right)=\int_{0}^{\infty} n \cdot r^{5} \cdot d r \\
M 4=\sum_{1}^{\infty} n \cdot r_{i}^{4} \quad M 5=\sum_{1}^{\infty} n \cdot r_{i}^{5} \\
M 4=4 \cdot r_{1}^{4}+10 \cdot r_{2}^{4}+6 \cdot r_{3}{ }^{4}+4 \cdot r_{4}^{4}+2 \cdot r_{5}^{4}+1 \cdot r_{6}^{4} \\
M 5=4 \cdot r_{1}{ }^{5}+10 \cdot r_{2}{ }^{5}+6 \cdot r_{3}{ }^{5}+4 \cdot r_{4}{ }^{5}+2 \cdot r_{5}{ }^{5}+1 \cdot r_{6}{ }^{5}
\end{gathered}
$$

The relationships among these moments represent different characteristics of the distribution. For example, $M_{3} / M_{2}$ represents the mean particle size based on the specific surface area, and is also known as the Sauter mean diameter (SMD) or $\mathrm{D}[3,2]$. Another moment mean that is broadly used is the volumetric mean particle size $D[4,3])$. Other moments are used to define mean particle sizes and particle size amplitudes, but it is important to highlight that the larger the moment order, the more it is influenced by bigger particles.

When static laser diffraction is used to monitor the particle size, optical models can be used to relate the interaction of a light beam with the particles (Mie, Rayleigh, and Fraunhofer). Mie (1908) developed a basic theory for spherical particles, which applies when particles with a wide range of sizes interact with light. Rayleigh's scattering theory applies when the particles are smaller than the wavelength of the 
incident beam. Fraunhofer's theory can be applied when the particles are substantially larger than the wavelength of the incident beam.

In the case of pigmentary $\mathrm{TiO}_{2}$, the particles are about half of the visible light wavelength range $(400 \mathrm{~nm}$ to $750 \mathrm{~nm})$. Therefore, the particles are in a range between $200 \mathrm{~nm}$ to $350 \mathrm{~nm}$. Sometimes bigger particles are found, due to the particular requirements of the application. Therefore, the Rayleigh-Mie solutions for Maxwell's equation are used to calculate the particle size based on light scattering.

According to the Rayleigh-Mie theory, the scattered light intensity (I) is independent of the particles' shape but takes into account the distance from particle to the detector $(r)$ the incident light intensity $\left(\mathrm{I}_{0}\right)$, and the wavelength $(\lambda)$ as in Equation 19:

$$
I=I_{0} \alpha^{2}\left(\frac{2 \pi}{\lambda}\right)^{4} \frac{1+\cos ^{2} \theta}{2 r^{2}}
$$

Equation 19

where $\alpha$ is the light polarization and is the parameter of the equation that contains the relative refractive index $(\mathrm{m})$ and the volume $(\mathrm{V})$ as presented in Equation 20:

$$
\alpha=\frac{3\left(m^{2}-1\right)}{m^{2}+2} \frac{V}{4}
$$

Equation 20

A simplified representation of a laser diffraction equipment is shown in Figure 16. The laser beam is emitted from the source passing throughout a series of lenses for conditioning, then through the sample suspension, in which the scattering takes place. The scattered light reaches the detectors, and the measured light intensity at different angles is processed by the computer software. 


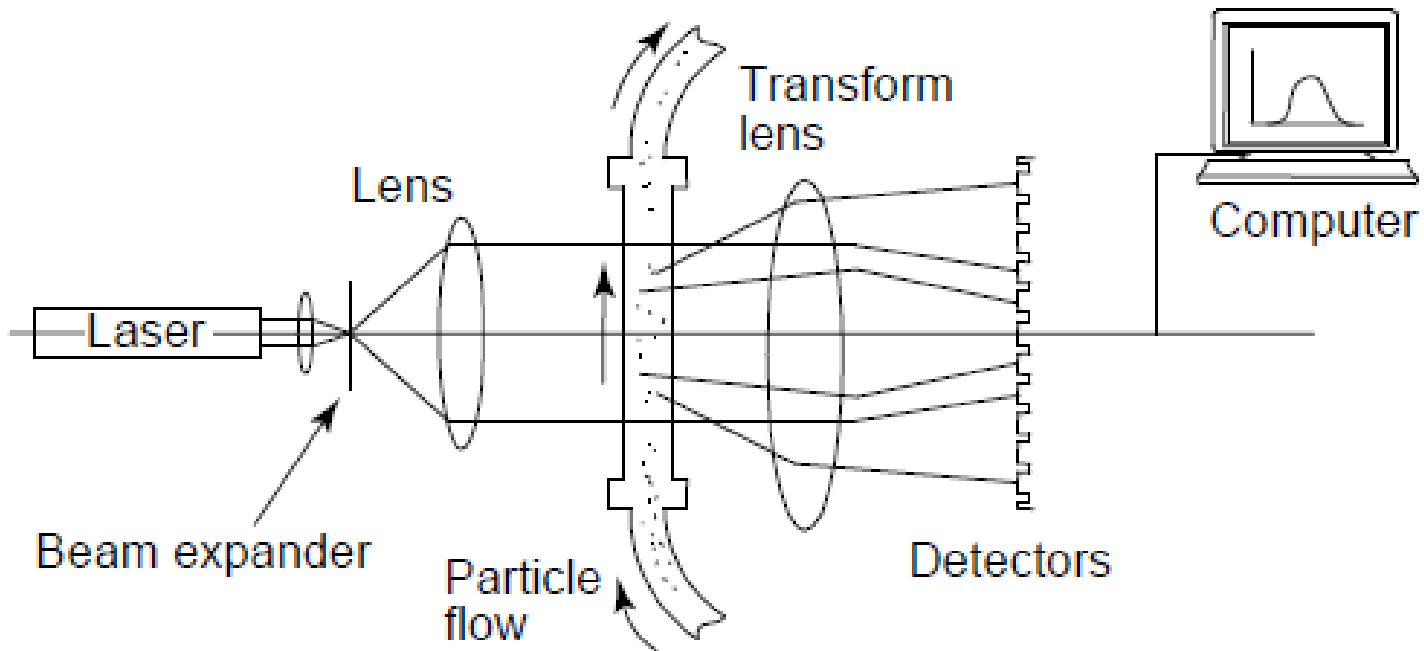

Figure 16 - A simplified scheme of a laser diffraction device for measuring particle size distribution

Additional relevant information gained from this kind of equipment is that obscuration or transmittance must be well controlled in order to prevent multiple scattering due to the interaction of the combined scattering of two or more particles, thus deviating from reliable information.

Figure 17 is a graphical description of different particles interacting with light as a function of the wavelength in terms of reflectance (Baneshi et al., 2009). In this study, the authors referred to reflectance when the light scatters back to the observer, which corresponds to the total backscattering by several particles. In this case, the authors used particles with controlled sizes. 


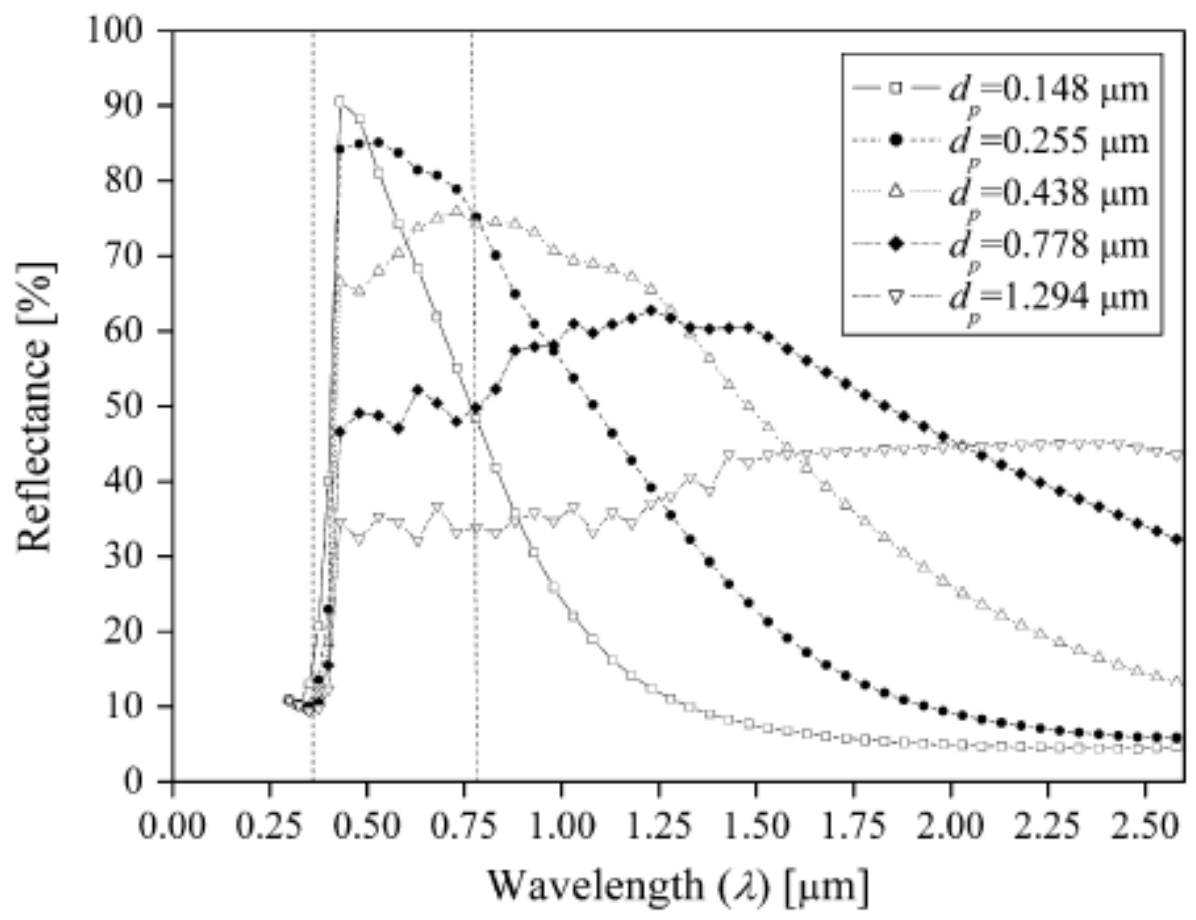

Figure 17 - Reflectance as a function of wavelength and particle size distribution. (Baneshi et all 2009)

\subsection{Surface Area}

As discussed in Chapter 2.2.1, the shape factor (I) may play an important role in wetting behavior. Therefore, the real surface area needs to be determined in order to calculate the relationship between the true surface area and the calculated area based on the assumption of a perfect spherical smooth-surface particle as presented in Equation 10.

$i=\frac{A_{i}}{A_{g}}$

Equation 10

Stephen Brunauer, Paul Hugh Emmett, and Edward Teller developed the BET measurement technique, which has become a standard procedure for the determination of the surface area of finely divided and porous materials.

Conceptually the technique is an extension of the Langmuir model, which considers that only one layer (monolayer) of the gas molecule is adsorbed onto the solid surface. In contrast, the BET model considers the formation of multilayers (Figure 18 ), in which the gas molecule is physically adsorbed onto the solid surface in 
several layers. The interaction among the layers is inexistent, and the Langmuir theory can be applied to each of the individual layers.

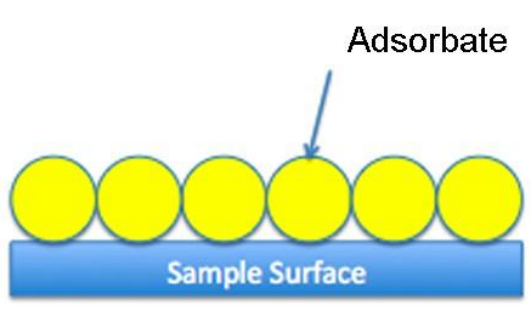

(a)

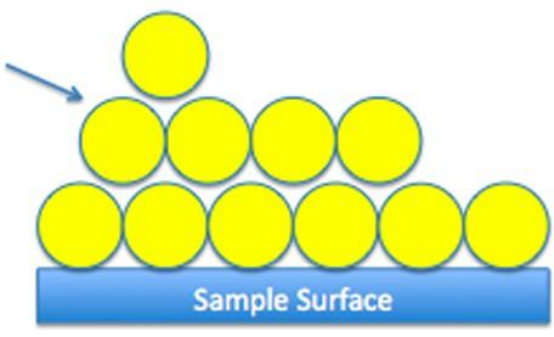

(b)

Figure 18 - The Langmuir (a) and BET (b) models.

The adsorption and desorption curves can be categorized into six different types, as in Equation 21.
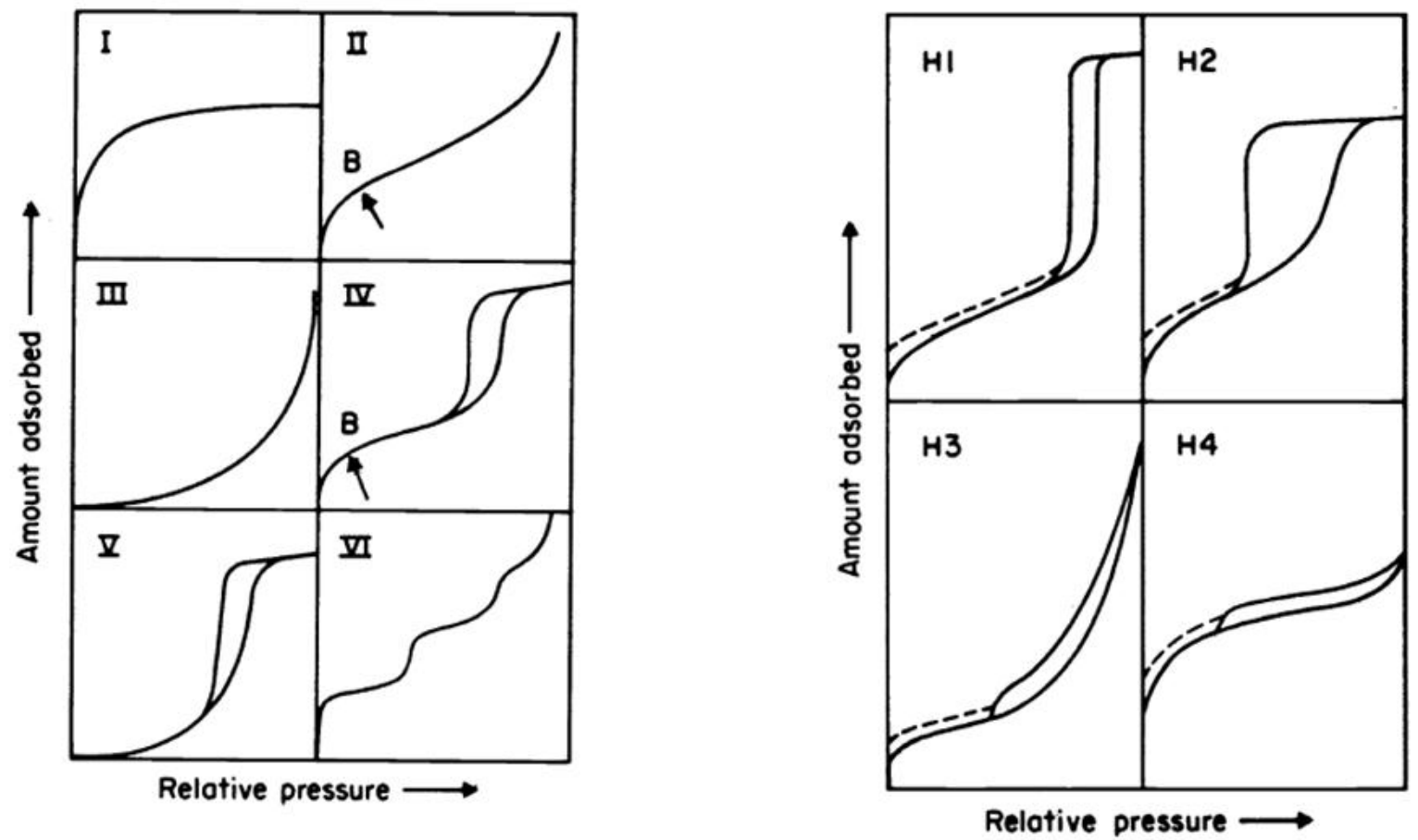

Figure 19 - Left: types of physisorption isotherms. Right: types of hysteresis loops (Sing et al., 1985)

Type I isotherm is concave to the relative pressure axis, and the adsorbed volume approaches a limiting value as the relative pressures reaches 1 . This isotherm is typically given by microporous solids with relatively small surfaces. The limiting uptake is governed by the accessible micropore volume and not by the internal surface area. 
Type II is usually obtained from a non-porous or macroporous adsorbent, representing no restrictions to the mono- and multilayer adsorption. Point $B$ is where the monolayer coverage is complete and the multilayer adsorption begins.

Type III is not a common curve, but a number of systems are described by it (i.e., nitrogen on polyethylene). It does not have a distinctive point $\mathrm{B}$, and the adsorbateadsorbate interactions play an important role.

Type IV presents a hysteresis loop and the limiting uptake over a range of large range of relative pressure. Type $\mathrm{V}$ isotherms are uncommon. They are related to type III isotherms in that the adsorbent-adsorbate interaction is weak, but it might be obtained with certain porous adsorbents.

Type VI isotherms represent stepwise multilayer adsorption on a uniform non-porous surface. The step height represents the monolayer volume for each adsorbed layer and remains nearly constant for two or three adsorbed layers.

Hysteresis appearing in the multilayer range of physisorption isotherms (type IV) is usually associated with capillary condensation on the mesopore structure. The step region of the desorption branch leading to the lower closure point occurs at a relative pressure, which is almost independent of the nature of the porous adsorbent, but it depends mainly on the nature of the adsorptive and its boiling point.

Having described the curves as well as the potential occurrence of hysteresis, the BET model can be mathematically represented by Equation 21 .

$\frac{V}{V_{\text {T:on }}}=\frac{c}{(1-z)\lceil 1-(1-c) z]}$

Equation 21

where $\mathrm{V}$ is the adsorbed gas volume, $\mathrm{V}_{\text {mon }}$ is the adsorbed gas volume considering a monolayer, $z$ is the relation between the equilibrium and saturation pressures ( $p$ and $p^{*}$, respectively) and $c$ is the BET constant expressed as Equation 22.

$c=\exp \left(\frac{E_{1}-E_{i}}{R T}\right)$ 
where $E_{1}$ is the first layer of heat adsorption, $E_{L}$ is the liquefaction heat of the adsorbate, $R$ is the ideal gas constant, and $T$ is the absolute temperature. The linearization of Equation 21 generates Equation 23, allowing determination of the adsorbate volume as a function of each applied pressure.

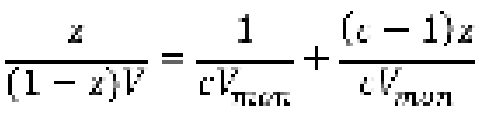

Equation 23

By plotting the left side of Equation 23 as a function of $z$, both $V_{\text {mon }}$ and the $C$ constant can be obtained. With this information, the powder specific area is obtained as well as the surface energy. Webb, 2003, associates the $c$ value to a measure of surface energy (analogous to surface tension of liquids). In the simplest approximation, Equation 24 is obtained.

"C" $\exp [(q 1-q L) / R T]$

Equation 24

where $\mathrm{q} 1$ is the heat of adsorption of the first layer of nitrogen on the solid surface and $\mathrm{qL}$ is the heat of liquefaction of nitrogen. Since $\mathrm{qL}$ is a constant, the logarithm, In( “C"), is proportional to q1. Since the adsorbant molecule is constant, q1 is thus dependent upon the surface energy of the $\mathrm{TiO}_{2}$ samples (actually the surface enthalpy).

The obtained surface area is considered the actual powder surface area or $A_{i}$ in Equation 10.

This leads to the missing information in Equation 10. $A_{e}$, the specific projected area of the particle can be obtained by calculating the sphere area with the radius equal to the average of the particle size, or by using the calculations of the particle size moments. The latter considers the distribution of the whole particle size and is therefore more accurate. The two methodologies are compared in Table 1, and the difference can reach $50 \%$. 
Table 1 - Comparison between the specific areas calculated based on the different methodologies described.

\begin{tabular}{lccccc}
\hline & A & A' $^{\prime}$ & A" $^{\prime \prime}$ & B & C \\
\hline D[3,2] $\mu \mathrm{m}$ & 0.40 & 0.42 & 0.78 & 0.39 & 0.33 \\
Specific area (Moments) $\mathrm{m}^{2} / \mathrm{g}$ & 2.5 & 2.4 & 1.3 & 2.6 & 3.0 \\
Specific area (D[3,2]) $\mathrm{m}^{2} / \mathrm{g}$ & 3.8 & 3.6 & 2.0 & 3.9 & 4.6 \\
Specific area (BET) $\mathrm{m}^{2} / \mathrm{g}$ & 11.3 & 10.4 & 11.2 & 21.0 & 19.2 \\
$\mathbf{i}($ BET/moments) & 4.6 & 4.4 & 8.7 & 8.2 & 6.4 \\
$\mathbf{i}^{\prime}($ (BET/D[3,2]) & 3.0 & 2.9 & 5.7 & 5.3 & 4.2 \\
Relation I//' & 1.54 & 1.54 & 1.54 & 1.54 & 1.54 \\
\hline
\end{tabular}

In this study, the curves are presented as shown in Figure 20, in which no significant hysteresis steps, neither microporous nor macroporous, are presented.

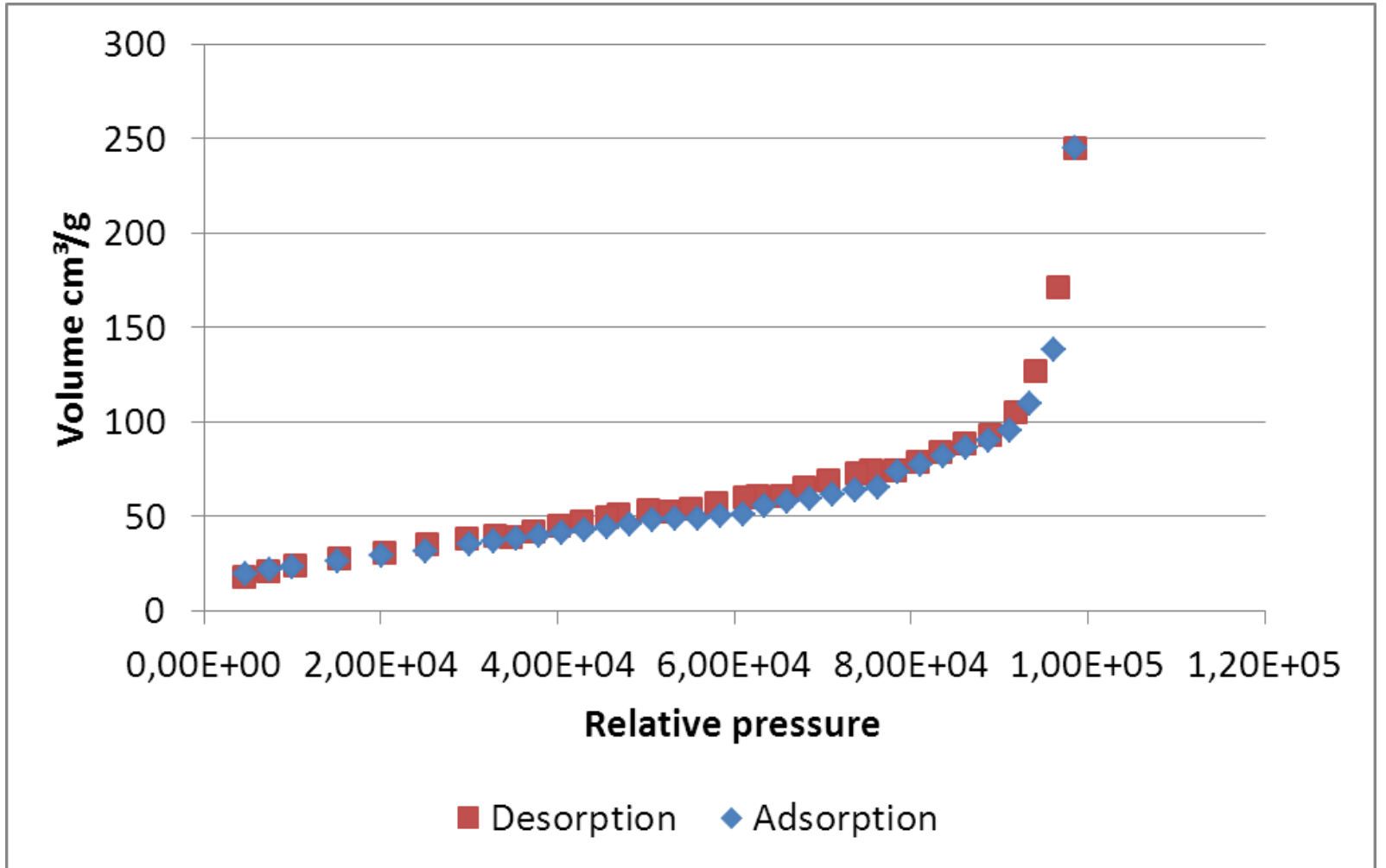

Figure 20 - Typical isotherm resulting from BET analysis, in this case, for grade B sample.

\subsection{Liquid surface tension}

Another important factor that influences speed is the surface tension of the penetrant liquid, which is formed by the unbalanced sum of forces in the surface of the material. The forces holding the particles of a condensed phase together become anisotropic in the phase boundary region (surface). Because an upper layer of molecules does not exist, a resultant force is created, which constantly pulls the 
molecules to the liquid bulk (Kosswig, 2000). The surface tension $(\gamma)$ for a given pressure $(P)$ and temperature $(T)$ are calculated based on Equation 25, where $G$ is the Gibbs free energy and $A$ is the surface area.

$\left(\frac{d G}{d i}\right)_{y, r}=\gamma$

Equation 25

Previous studies have established and tabulated the values for pure liquids. The CRC (Chemical Rubber Company) Handbook of Chemistry and Physics (92nd edn, 2011-2012) provides the values for a few pure liquids that were used in this study. These are shown in Table 2.

Table 2 - Surface tension of pure liquids used in this study

\begin{tabular}{ccc}
\hline Liquid & $\begin{array}{c}\text { Temperature } \\
\left({ }^{\circ} \mathbf{C}\right)\end{array}$ & $\begin{array}{c}\text { Surface tension }(\gamma) \\
\mathrm{mN} / \mathrm{m}\end{array}$ \\
\hline Water & 25 & 72 \\
n-Hexane & 25 & 18 \\
Ethanol solution (40\%) & 25 & 30 \\
\hline
\end{tabular}

As seen in Equation 9, section 2.2.1, the surface tension is directly responsible for changes in the wetting speed of the agglomerates as well as the capillary pressure that is presented in Equation 7 in the same section. In these equations, the smaller the tension is, the greater the wettability of the fluid and water. From this perspective, it is the liquid with most difficult relative wettability. As an illustration of practical implications, about $60 \%$ of the world's $\mathrm{TiO}_{2}$ production is applied to coatings, and most companies in the coating industry apply $\mathrm{TiO}_{2}$ to waterborne coatings, although a few solvent-borne formulas are being converted into water-based systems. Therefore, the coating formulators have to use additives to change the surface tension of the water to make the system more amenable to wetting. The surfactants in use by the coating industry are usually a sodium salt of a maleic anhydride copolymer-based product.

The response of the surface tension of water at $50{ }^{\circ} \mathrm{C}$ to the addition of a surfactant compound (sodium octyl benzene sulphonate/nonyl phenol ethoxylate) is typically represented by a curve, as shown in Figure 21, where the surface tension is reduced 
to a minimum value and characterized by the surfactant-liquid (water in this case) interaction.

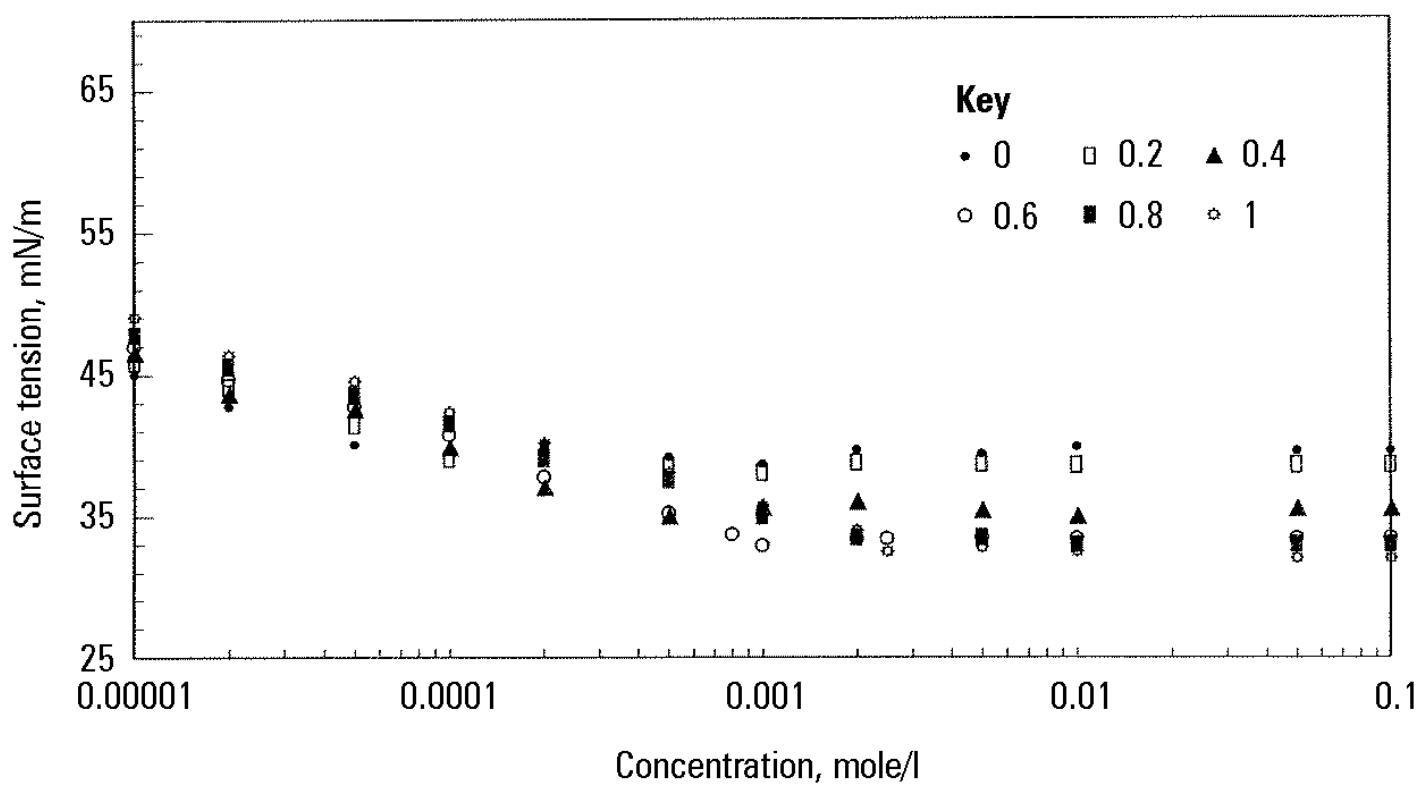

Figure 21 - Surface tension of water at $50{ }^{\circ} \mathrm{C}$ obtained for various concentrations of water mixed with a generic surfactant solution with different mole fractions (Hamid; Omar, 1999).

The minimum value occurs mainly because the surfactant molecules are usually formed by a hydrophobic and a hydrophilic function, which are also referred to as a tail and a head, respectively. In highly diluted aqueous solutions, surfactants exist in in monodisperse form and are concentrated at the interface oriented towards the hydrophilic (or head) immersed in the water. The presence of the surfactant at the liquid surface reduces the Gibbs free energy, thus reducing the surface tension.

\subsection{Work of dispersion}

With the information about the liquid surface tension, the solid surface tension is calculated by the capillary rise (Washburn) method. Depending on the roughness of the particle, it is possible to calculate the work of dispersion of each particle in relation to each dispergent medium.

The work of dispersion is calculated by the sum of the work of adhesion, $W_{a}$, the work of penetration, $\mathrm{W}_{\mathrm{p}}$, and the work of spreading, $\mathrm{W}_{\mathrm{s}}$. These works rule the wetting of a particle. When the work of dispersion is positive, the wetting is spontaneous. 
The work of adhesion occurs during the first moment when the particle is brought into face-to-face contact, and the liquid starts wetting the particle by adhering to the particle surface. It can be calculated by Equation 26:

$W_{c}=\gamma_{L}(i \cos \theta+1)$

Equation 26

where $W_{a}$ is the adhesion work, $\gamma_{L}$ is the liquid surface tension, $i$ is the particle roughness and $\theta$ is the contact angle between the liquid and the solid.

The work of penetration is generated by the liquid while it penetrates the solid pores or solid surface roughness. It can be calculated by Equation 27:

$W_{p}=\gamma_{L}(i \cos \theta)$

Equation 27

where $W_{p}$ is the penetration work.

Finally, the work of spreading refers to a situation when the liquid flows over a surface as a duplex film and not as a monomolecular layer. This layer is thick enough to consider that the two interfaces are independent in their surface characteristics. This work is calculated based on Equation 28:

$W_{s}=\gamma_{L}(i \cos \theta-1)$

Equation 28

where $\mathrm{W}_{\mathrm{s}}$ is the spreading work.

In summary, the factors affecting the required energy to achieve maximum dispersion in this study are presented in Figure 22. 


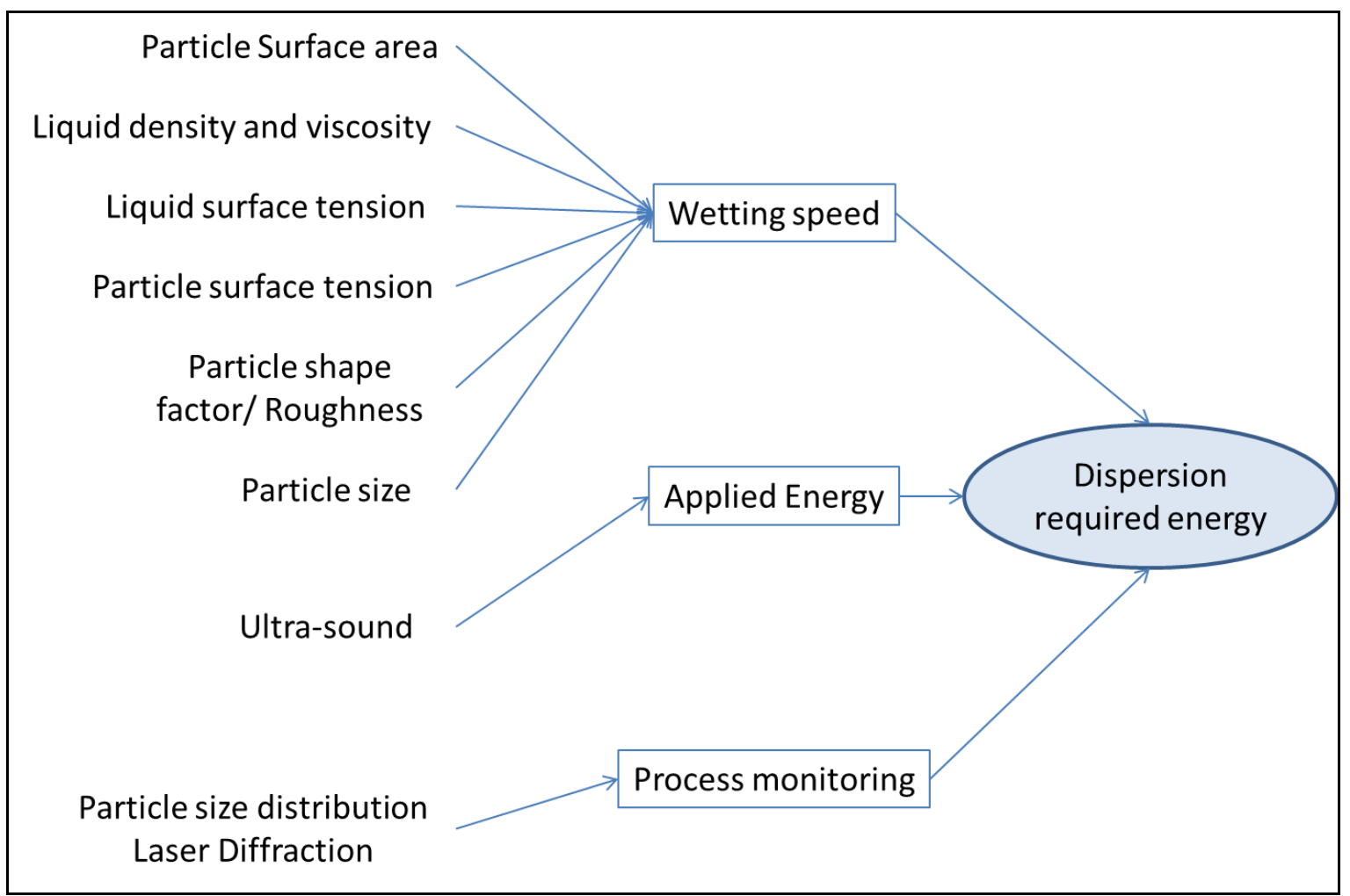

Figure 22 - Graphical summary of the properties affecting the energy required for dispersion 


\section{Materials and Methods}

\subsection{Samples}

The study consists of a number of laboratory scale experiments with five samples of pigment grade $\mathrm{TiO}_{2}$ particles and three main liquids, $\mathrm{n}$-hexane, ethanol and water. The surface tension of the water was changed by the addition of different values of a surfactant before mixing with ethanol in a mass proportion of $20 \%$ ethanol and $80 \%$ water. Table 3 shows the surface tension of the liquids and where they were used in this study; this group of liquids was used to provide a broad range of surface tensions.

Table 3 - Surface tension of the pure liquids and different formulations.

\begin{tabular}{|c|c|c|c|c|c|c|c|}
\hline Liquid & N-hexane & $\begin{array}{c}\mathbf{2 0 \%} \text { ethanol } \\
\mathbf{8 0} \% \text { water }\end{array}$ & Water & $\begin{array}{c}\text { Water + 0,50\% } \\
\text { surfactant }\end{array}$ & $\begin{array}{c}\text { Water + 1,00\% } \\
\text { surfactant }\end{array}$ & $\begin{array}{c}\text { Water + 1,50\% } \\
\text { surfactant }\end{array}$ & $\begin{array}{c}\text { Water + 3,00\% } \\
\text { surfactant }\end{array}$ \\
\hline $\begin{array}{c}\text { Surface } \\
\text { tension } \\
(\mathrm{mN} / \mathrm{m})\end{array}$ & 18 & 30 & 73 & 59 & 54 & 51 & 45 \\
$\begin{array}{c}\text { Used in } \\
\text { Washburn } \\
\text { analysis }\end{array}$ & Yes & Yes & Yes & Yes & Yes & Yes & Yes \\
\hline $\begin{array}{c}\text { Used in } \\
\text { PSD } \\
\text { analysis }\end{array}$ & No & No & Yes & Yes & Yes & Yes & No \\
\hline
\end{tabular}

All water-based solution samples were prepared using a volumetric flask and analytical scales in order to maintain the precision and reproducibility of the solution preparations. The volumes used in each analysis were prepared on the day they were used. The only sample that did not require preparation was n-hexane.

\subsubsection{Titanium Dioxide}

The titanium dioxide particle samples used in this study were selected in order to cover different particle size ranges with similar surface properties, as well as similar particle sizes with different surface properties. Although detailed information on how the samples were produced was not available, all samples are rutile $\mathrm{TiO}_{2}$ produced by the chloride process, and the characterization of the samples is shown in Table 4. 
Table 4 - Particles properties

\begin{tabular}{|l|ccccc|}
\hline Property/Sample & A & A' $^{\prime}$ & A" $^{\prime \prime}$ & B & C \\
\hline Particle $r(\mu \mathrm{m})$ & 0.22 & 0.26 & 0.35 & 0.22 & 0.21 \\
C $($ BET $)$ & 43 & 46 & 41 & 50 & 28 \\
Specific area BET $\left(\mathrm{m}^{2} / \mathrm{g}\right)$ & 11.3 & 10.4 & 11.2 & 19.2 & 22.0 \\
Specific area Moments $\left(\mathrm{m}^{2} / \mathrm{g}\right)$ & 2.5 & 2.4 & 1.3 & 2.6 & 3.0 \\
Average of pore volumes $(\mathrm{mL})$ & 3.3 & 3.2 & 2.4 & 2.3 & 2.1 \\
i (BET/Mon) & 4.6 & 4.4 & 8.7 & 7.5 & 7.3 \\
\hline
\end{tabular}

Grade A was produced using different levels of micronization to generate different particle sizes of the same grade. The regular size was $A$; the bigger particles ( $A^{\prime}$ and A") were especially produced and are not currently commercially available to the market.

\subsubsection{Surfactant}

The surfactant used in this study was Orotan $731 \mathrm{~A}$, which is actually a dispersant widely used in the coatings industry. It is a hydrophobically modified sodium salt of a carboxylate polyelectrolyte. The surfactant properties are shown in Table 5.

Table 5 - Surfactant properties

\section{Property}

\begin{tabular}{ll}
\hline Appearance & Clear yellow liquid \\
\hline pH & 9.5 to 10.5 \\
\hline Solids, by weight, (\%) & 25 \\
\hline Density (g/ml) & 1.10 \\
\hline
\end{tabular}

\subsection{6. n-Hexane}

The source of the N-hexane used in this study was Vetec Química Fina. The properties are shown in Table 6. 
Table 6 - n-hexane typical properties (Vetec)

\begin{tabular}{ll}
\hline Property & Value \\
\hline Purity (Isomers mix) & Min. 99.9\% \\
\hline Purity (n-hexano) & Min. 96\% \\
Water & Max. 0.01\% \\
\hline Isomers content & Max. 3.9\% \\
Non volatile & Max. 0.0002\% \\
Epoxy heptachlore content & Max.5 mg/L \\
2-octanol content & Max. $5.0 \mu \mathrm{g} / \mathrm{L}$ \\
\hline
\end{tabular}

\subsubsection{Ethanol}

The source of the ethyl alcohol (ethanol) used in this work was Vetec Química Fina. The properties are presented in Table 7.

Table 7 - Typical properties of ethyl alcohol (Vetec).

\begin{tabular}{ll}
\hline Property & Value \\
\hline Purity & Min. 99.8\% \\
\hline Non volatile content & Max. 0.0005\% \\
\hline Water (Karll Fisher) & Max. 0.2\% \\
\hline Acidity (as CH3COOH) & Max. 0.001\% \\
\hline Free Alkali (as NH3) & Max. 0.0003\% \\
\hline
\end{tabular}

\subsubsection{Water}

The water used in this study was tap water that was deionized and sterilized using Elix $^{\circledR} 100$ (Millipore) equipment, which contained a system of reverse osmosis, an ultraviolet lamp, and an ion exchange column. The typical properties of the water obtained from the deionization equipment are shown in Table 8.

Table 8 - Typical properties of deionized water (Millipore).

\begin{tabular}{ll}
\hline Property & Value \\
\hline Resistivity & $>5 \mathrm{M} \Omega . \mathrm{cm}$ (typically 10 to $15 \mathrm{M} \Omega . \mathrm{cm}$ ) \\
Conductivity & $<2 \mu \mathrm{S} / \mathrm{cm}$ (typically 0.067 to $0.10 \mu \mathrm{S} / \mathrm{cm}$ ) \\
Typical Total Organic Carbon & $<30 \mathrm{ppb}$ \\
Bacterial Count & $<10$ Colony forming unit/mL \\
\hline
\end{tabular}




\subsection{Methods}

\subsubsection{Particle size distribution}

A Malvern Mastersizer $X$ laser diffractometer with a sampling unit equipped with agitation and a sonication was used to monitor the particle size distribution of the samples in the dispersion experiments. The equipment is based on the angular distribution of forward scattered light, operates with a collimated laser beam at 640 $\mathrm{nm}$ and is able to provide the particle size distribution in the range from 0.1 to 600 $\mu \mathrm{m}$. Although different optical models can be adopted to estimate the particle sizes, in the present study the Mie model was adopted.

To generate the shear that provides the energy to break the agglomerates into aggregates and individual particles, a high-energy ultrasound generator was used (UP100H Ultrasonic Processor, Hielscher). The output of this equipment, as specified by the supplier, is $100 \mathrm{~W}$ at $30 \mathrm{kHz}$, which can be adjusted for better control of the energy applied to the suspension down to $20 \%$ of its full power.

Each powder sample (each of the different grades of $\mathrm{TiO}_{2}$ ) was added to the sampling unit containing the liquid with the desired surface tension (water + dispersant) under constant agitation until the obscuration rate measured by the laser diffractometer attained 10\% (the minimum operational obscuration rate required). Sonication promoted deagglomeration, and the dispersed powder scattered more light, thus increasing the obscuration rate as illustrated in Figure 23. At the end of the procedure, the obscuration rate was close to the upper operational limit of the equipment (30\%). 


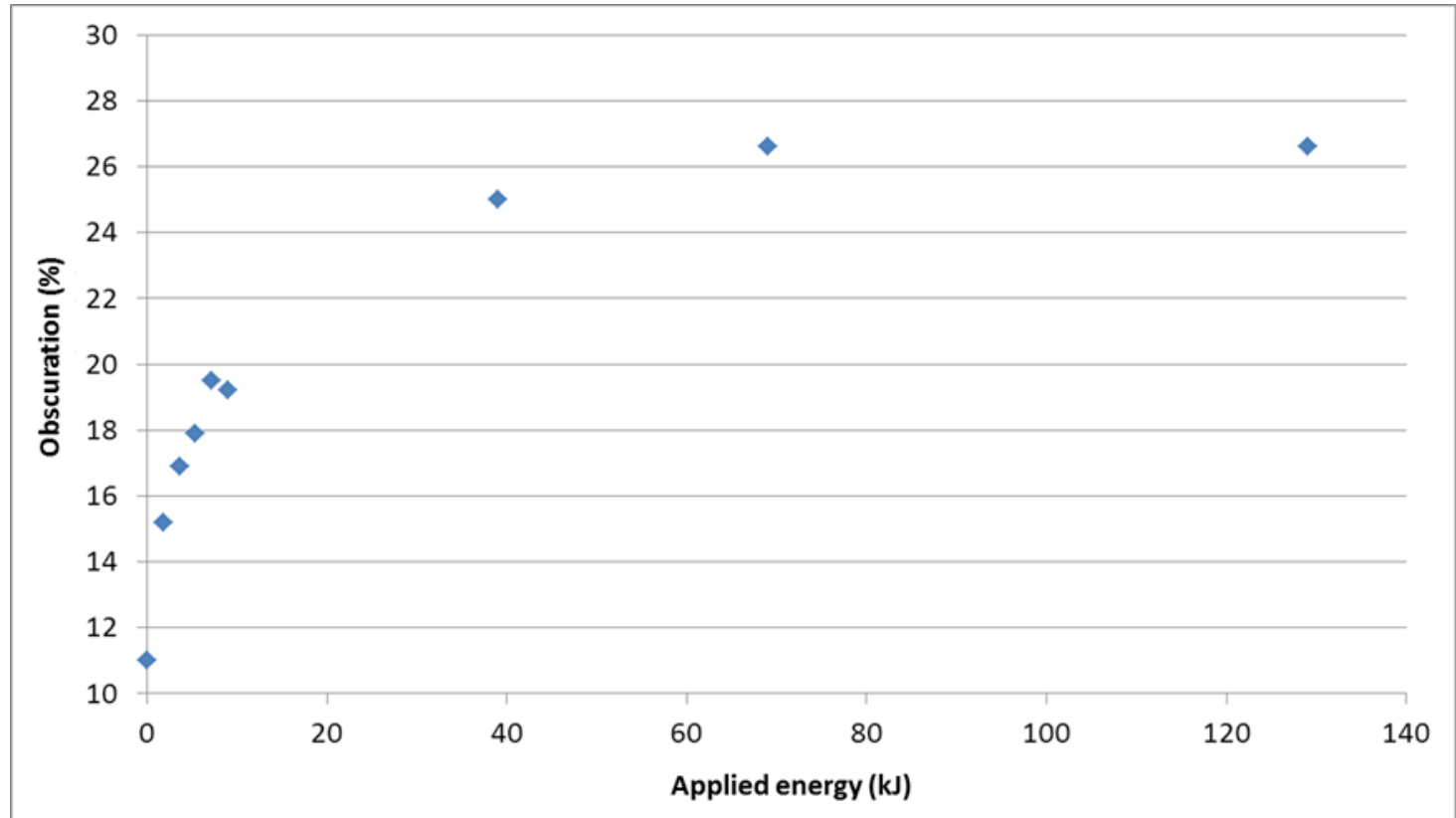

Figure 23-Obscuration rate as a function of the energy applied for sample C.

Agitation in the sampling unit was adjusted in order to keep the powder from settling (with the suspension as homogeneous as possible), without applying too much shear in order to avoid the breakage of agglomerates. The recirculation rate was also controlled in order to reduce the shear as much as possible.

This analysis enabled the monitoring of the particle size distribution over time, or as a function of the energy applied. Typical curves of particle size distribution as a function of the applied energy are presented in Figure 24, showing that the size distribution curves become narrower as a function of the applied energy and the destruction of larger agglomerates. 


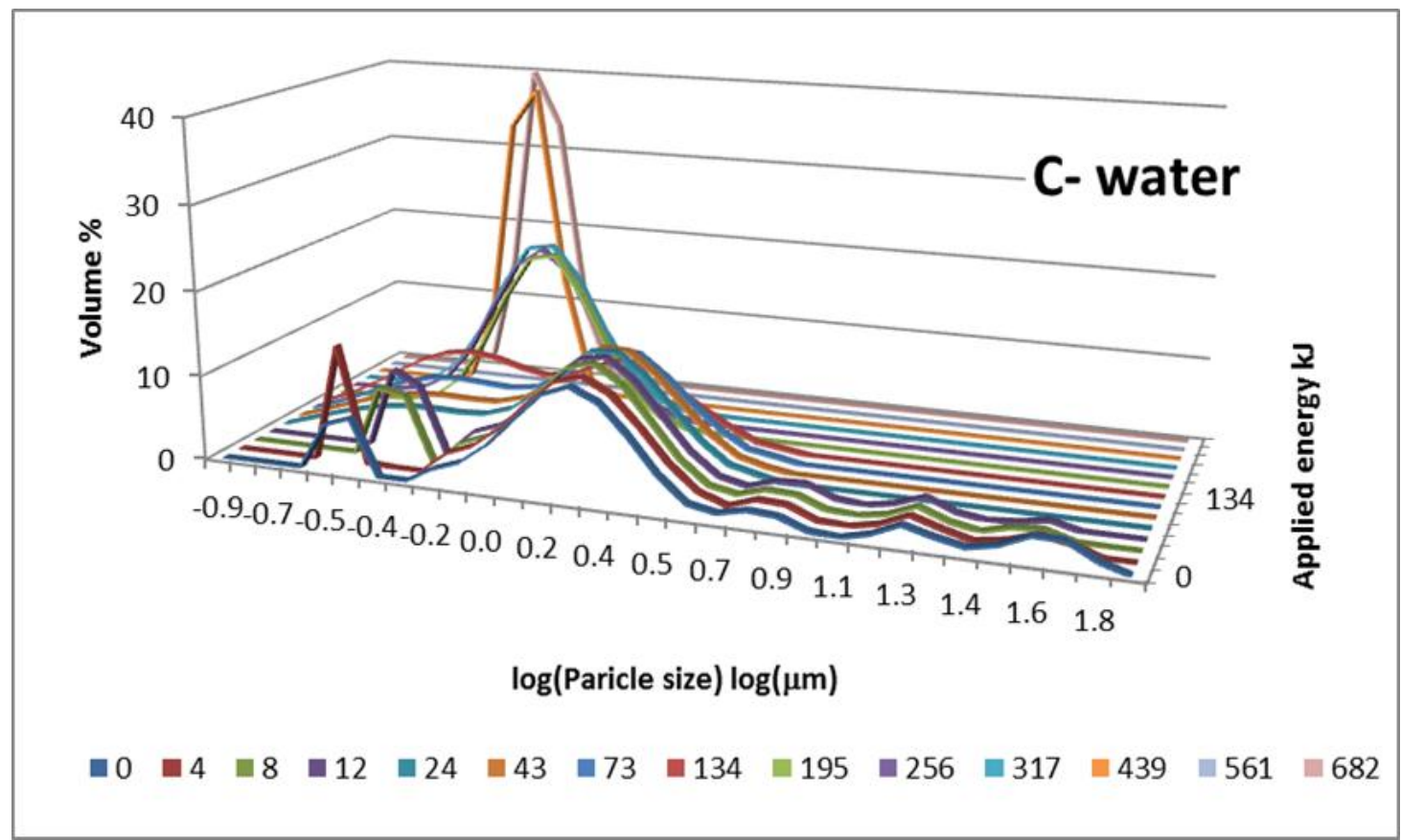

Figure 24 - Particle size distribution as a function of the applied energy for sample C dispersed in DI water.

The changes in particle size distribution were also monitored by calculating the surface-volume (Sauter) mean diameter, $\mathrm{D}(3,2)$, and the volumetric mean diameter, $\mathrm{D}(4,3)$. These diameters are calculated by the ratio of two different moments to represent different mean particles of the distribution.

The incorporation of the particles into the liquid medium under the action of ultrasound was carried out in order to estimate the effect of the controlled shear stress applied by the ultrasound generator on the particle size distribution. The energy applied can then be quantified (output power multiplied by the time in seconds), and a correlation between the energy applied and the parameters of the particles and the liquid can be obtained.

The sampling unit of the laser diffraction equipment was used as a mixing and dispersion recipient for the dispergent liquid medium and the particles. The sonication process was also carried out in the same sampling unit in order to avoid sampling of the suspension. To control the energy applied during sonication, the ultrasound power output was gradually increased, starting at $20 \%$ of the maximum level and achieving $100 \%$. The design of the sonication experiments is shown in Table 9 and in Figure 25. 
Table 9 - Sonication procedure for the samples in this study

\begin{tabular}{ccc}
\hline $\begin{array}{c}\text { Accumulated } \\
\text { Time (s) }\end{array}$ & $\begin{array}{c}\text { Power output } \\
\text { percentage } \\
(\%)\end{array}$ & $\begin{array}{c}\text { Applied } \\
\text { Energy } \\
\text { (kJ/L) }\end{array}$ \\
\hline 0 & 0 & 0 \\
10 & $20 \%$ & 4 \\
20 & $20 \%$ & 8 \\
30 & $20 \%$ & 12 \\
60 & $30 \%$ & 24 \\
90 & $50 \%$ & 43 \\
120 & $100 \%$ & 73 \\
150 & $100 \%$ & 134 \\
180 & $100 \%$ & 195 \\
\hline 210 & $100 \%$ & 256 \\
240 & $100 \%$ & 317 \\
300 & $100 \%$ & 439 \\
360 & $100 \%$ & 561 \\
\hline 420 & $100 \%$ & 682 \\
\hline
\end{tabular}

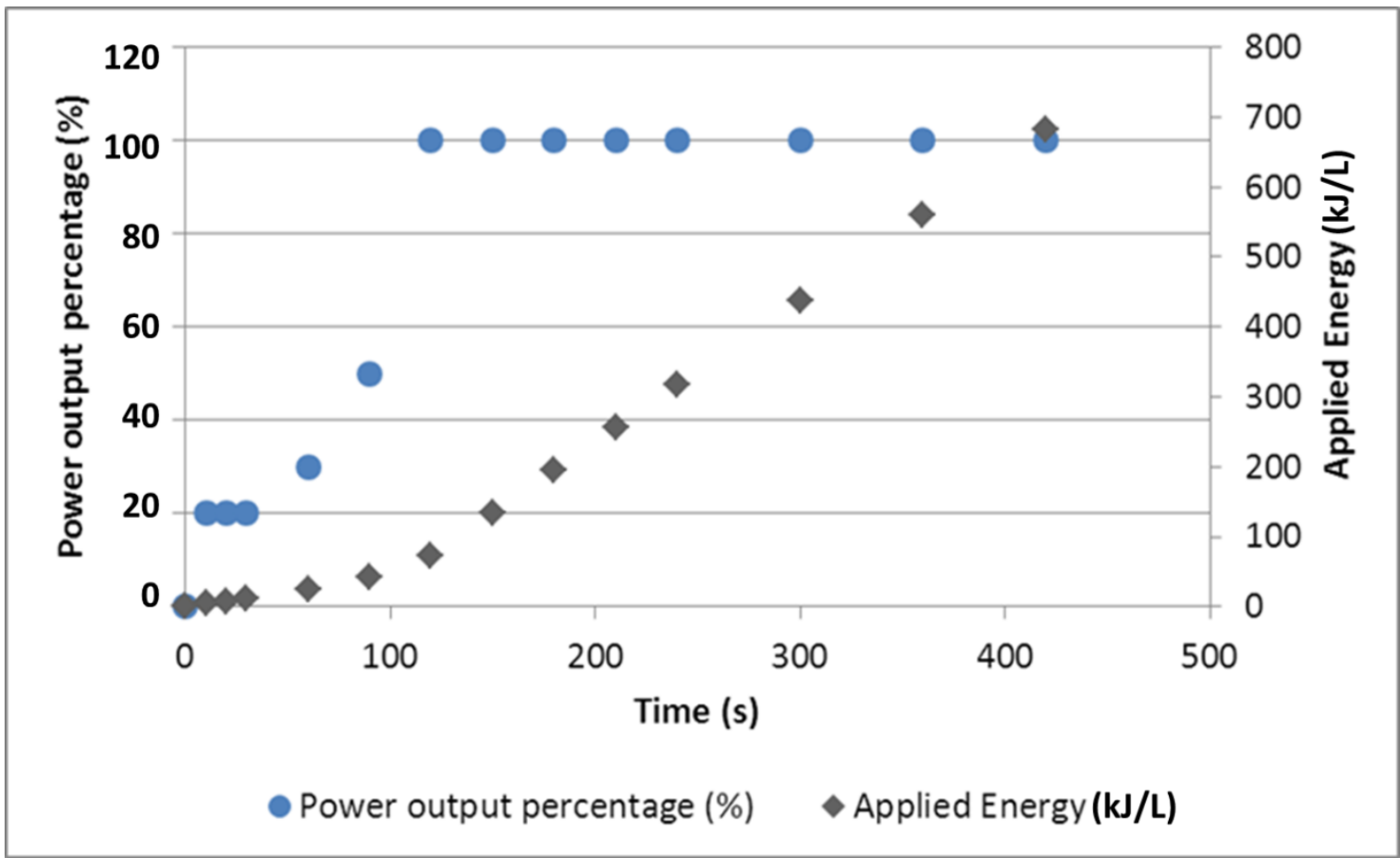

Figure 25 - Output percentage and applied energy as a function of time for the ultrasound generator.

Figure 26 and 28 illustrate the behavior in a typical analysis. As shown in Figure 27, the volumetric mean particle size, $D(4,3)$, is reduced as the energy is applied through sonication, achieving a minimum size at ca. $250 \mathrm{~kJ}$. However, $\mathrm{D}(3,2)$ shows a different behavior, which is caused by changes in the shape of the particle size distribution curve. The first $\mathrm{D}(3,2)$ minimum is achieved at about $150 \mathrm{~kJ}$ (point 1). 
However, $\mathrm{D}(4,3)$ did not reach the minimum value at the same energy value. At point 2 , $D(3,2)$ sloped up while $D(4,3)$ continued to decrease. In the following sonication step (point 3), both minima were achieved.

In Figure 27 the horizontal axis is in logarithmic scale in order to evidence the longer term tendency. The slope between points 1,2 and 3 results from the deagglomeration of large groups of particles (see Figure 25). Until this point, the particle size distribution is bimodal, formed by larger agglomerates and individual agglomerates. From point 1 to point 2, the second peak of the distribution is completely destroyed, generating a single peak that is broader than the previous ones. This broader distribution results in a slightly higher volume mean particle diameter in point 2 than in point 1.

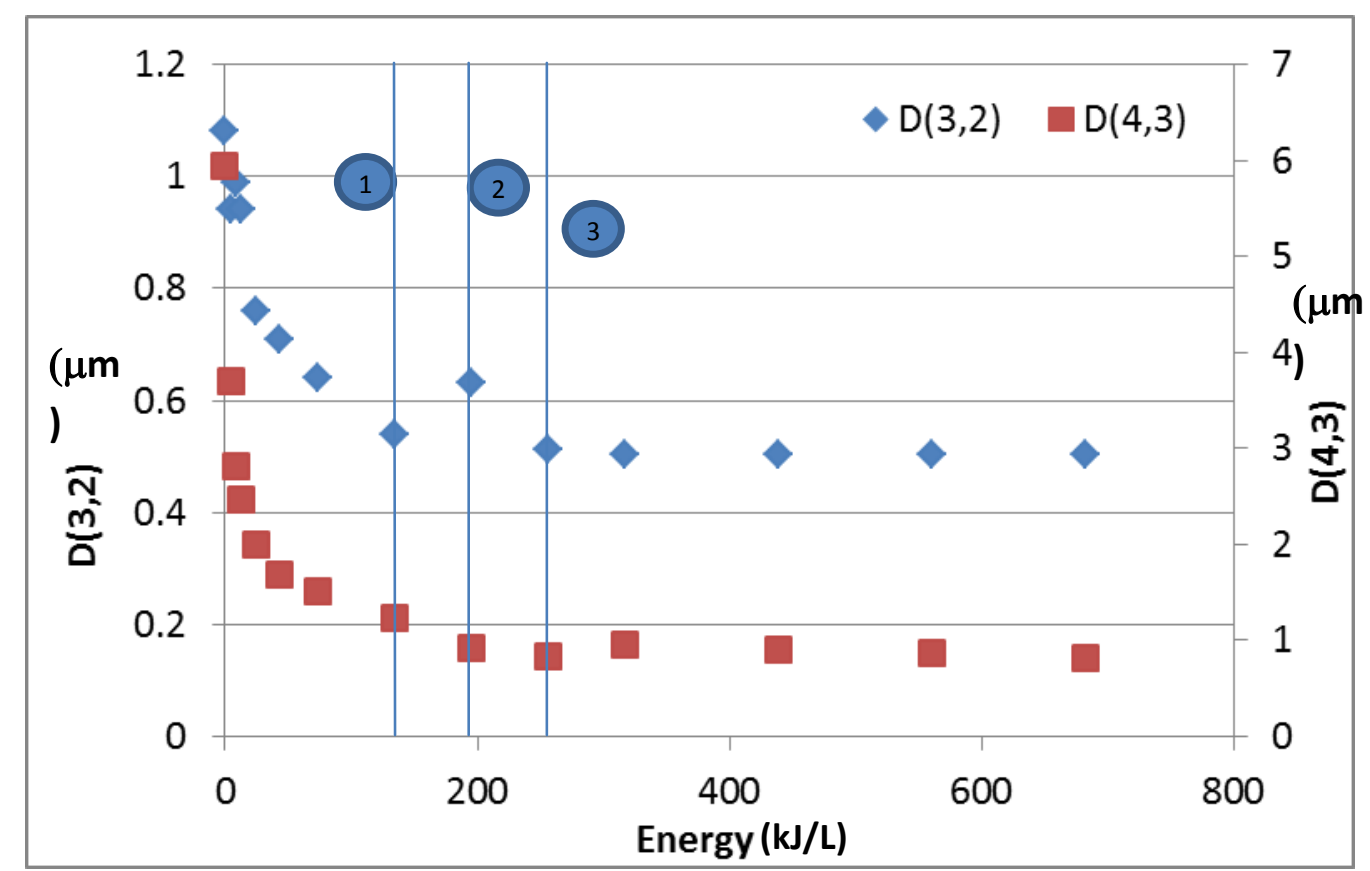

Figure 26 - Mean particle sizes $D(3,2)$ and $D(4,3)$ as a function of the required energy for particle "C" dispersion. 


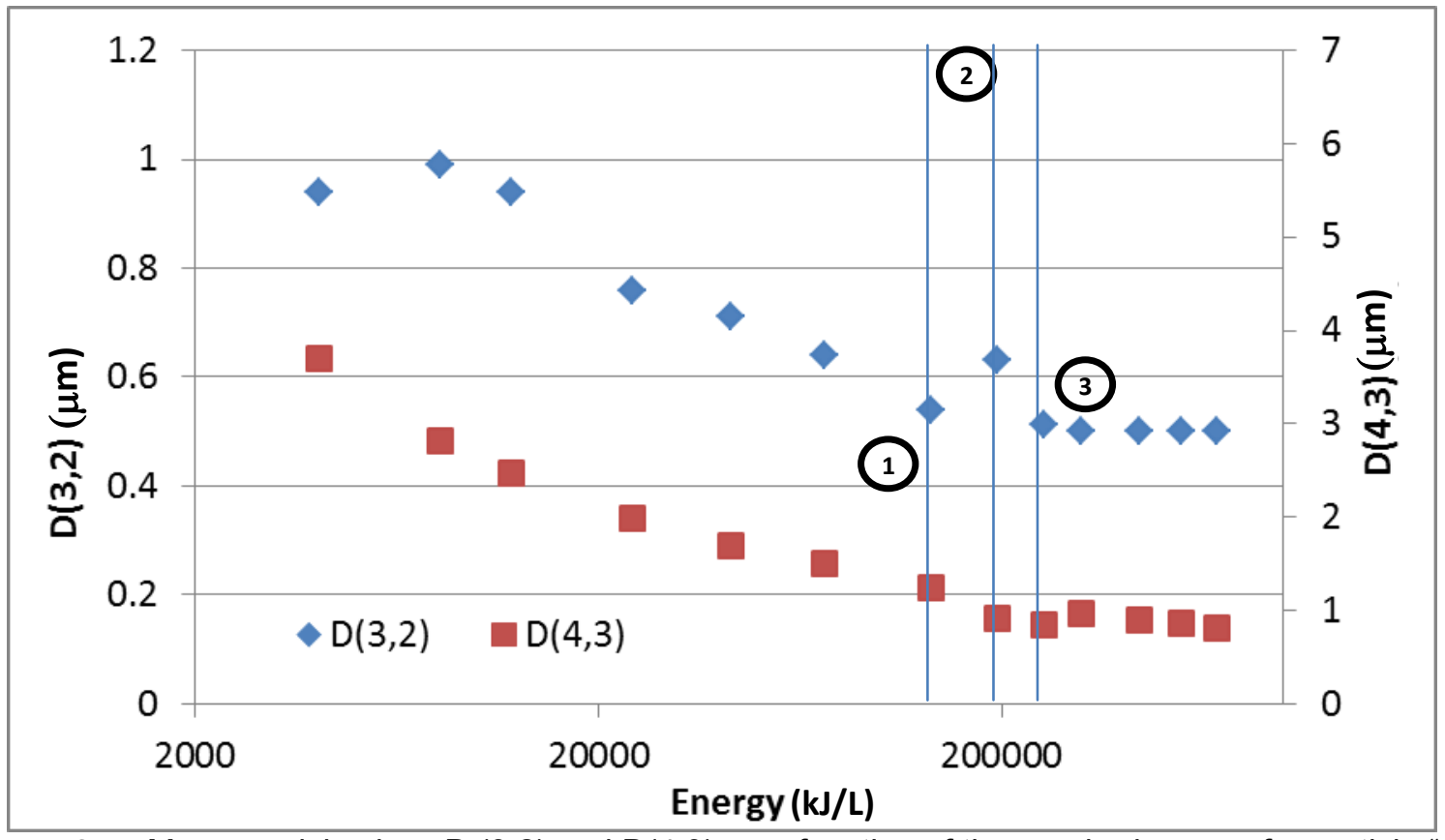

Figure 27 - Mean particle sizes $D(3,2)$ and $D(4,3)$ as a function of the required energy for particle " $C$ " dispersion.

The effect presented above can be better explained by the Figure 28 .

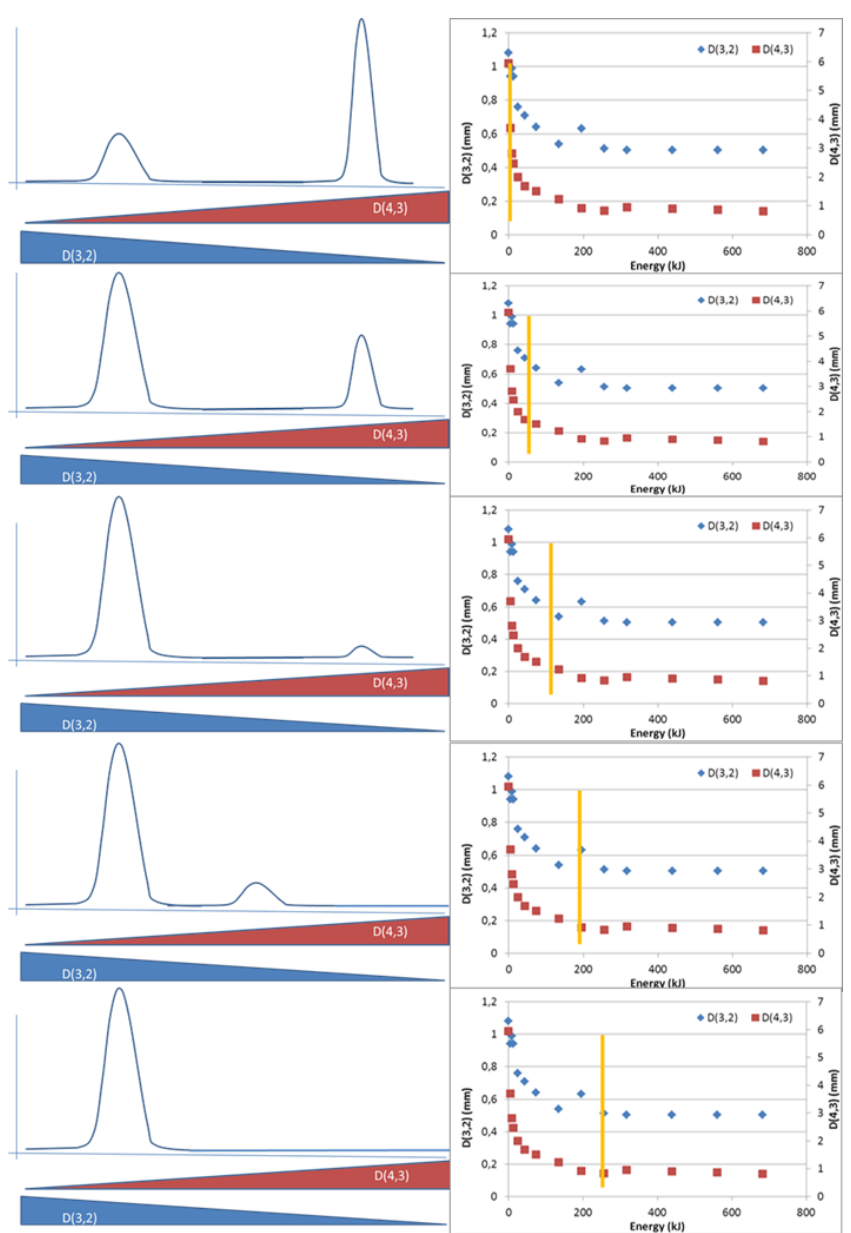

Figure 28 - evolution of $D(3,2)$ and $D(4,3)$ as a function of particle size distribution. 


\subsubsection{Surface area}

The measurement of the surface area by BET methodology was applied using the NOVA 1200e surface area \& pore size analyzer (Quantachrome Instruments). The information extracted from this equipment allows the evaluation of the precise value of the total surface area in $\mathrm{m}^{2} / \mathrm{g}$. When the $\mathrm{TiO}_{2}$ particle itself is not porous, as shown in Figure 1, changes in surface area should denote the level of roughness of these solids and/or the shape factor of the particle.

As discussed in Chapter 2.2.1, Equation 3 and Equation 2 are both affected by the surface area, the former by changes occurring in the shape factor and the latter by the coordination number, which can also be affected by the shape factor.

Figure 29 shows an attempt to put into perspective one of the effects of the surface area on particle-to-particle interaction. The sphere is the lowest area per volume particle shape, and the accommodation between particles is the most compact achievable. As the surface becomes rougher, the space between the particles becomes more significant, and the particle-to-particle contact areas become smaller. The other effect is that, as depicted in Figure 30, where the particle surface is larger than the spherical ones (but differently than in Figure 29), the shapes better accommodate and reduce the spaces among them. Therefore, particle surface area is a good indication that the surface is different, but it does not give its direction (Figure 29 or Figure 30).
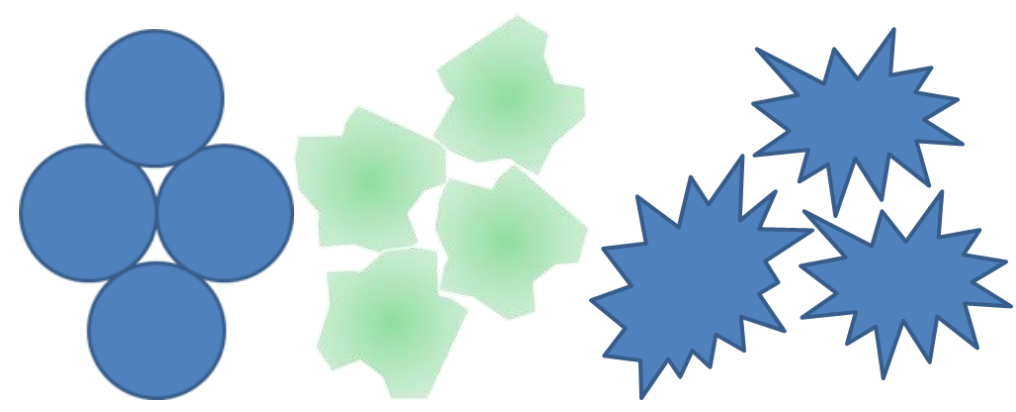

Figure 29 - Illustration of different shape factors for particles with rougher surfaces than a spherical particle 


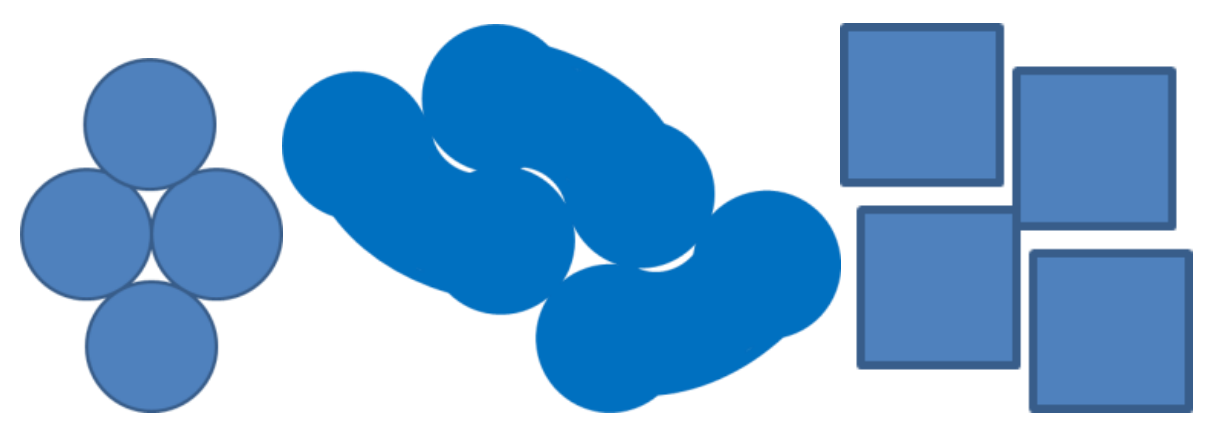

Figure 30 - Illustration of different shape factors for particles with smooth surfaces but of different shapes than a spherical particle

It is clear that the broader the distance between the particles, the larger amount of free space is available for the liquid to flow into the agglomerates and promote the spreading of the particles. Therefore, another test should be carried out to determine the direction in which the increased surface area is heading.

The samples were submitted to pre-treatment, which is required to remove impurities from the surface that could give mistaken information from the surface area. By not letting the nitrogen condensate on the surface of the solid, these impurities can be adsorbed, such as water, volatile organic compounds, or other compounds that are adsorbed by the surface but it are not part of it. The sample is gradually heated; the standard heating treatment is to submit the sample to a temperature of $200{ }^{\circ} \mathrm{C}$ for three hours. After the pre-treatment, the samples are transferred to the measuring chamber of the equipment, where the sorption and desorption occurs, as described in Chapter 2.4.

\subsubsection{Wetting tests}

The wettability of the different samples was estimated by capillary rise, based on the Washburn methodology. The equipment was a K100 (KRÜSS) with a fiber chamber (SH0620) $12 \mathrm{~mm}$ in inner diameter. This chamber was filled with 3 to $3.5 \mathrm{~g}$ of the powder used in the study. Several packing processes were studied to define the best reproducible method.

The equipment was used to evaluate the amount of liquid mass adsorbed over a given time. The adsorption speed is a function of several factors, as presented in Chapter 2.2.1, but this method provides information on capillarity adsorption, solidliquid affinity, solid surface tension, and capillary volume. 
Since this methodology is highly dependent on the powder packing factor, the sample preparation needs to ensure that the powder is compressed in the same way in order to pack the sample in a reproducible manner. In this study, the sample preparation evolved from taps alone to taps and hold screw revolutions (Figure 31 and Figure 32). The best reproducibility was 10 taps and 26 revolutions.

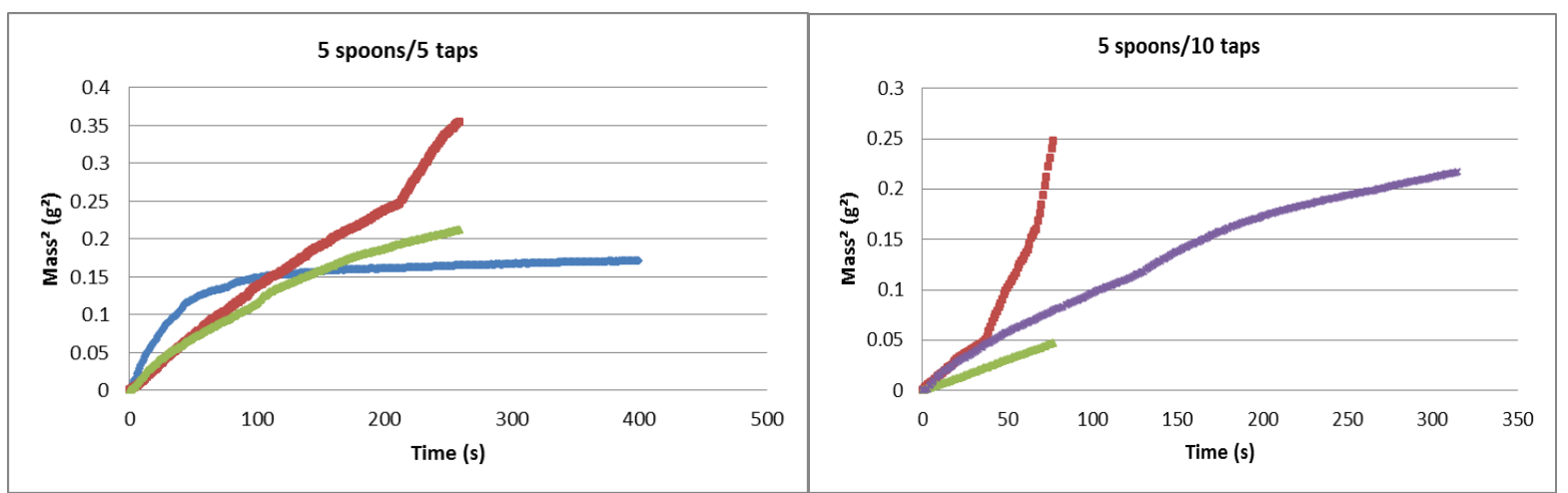

Figure 31 - Washburn methodology adaptation: influence of the tapping method during sample preparation into the repeatability and reproducibility of the methodology.

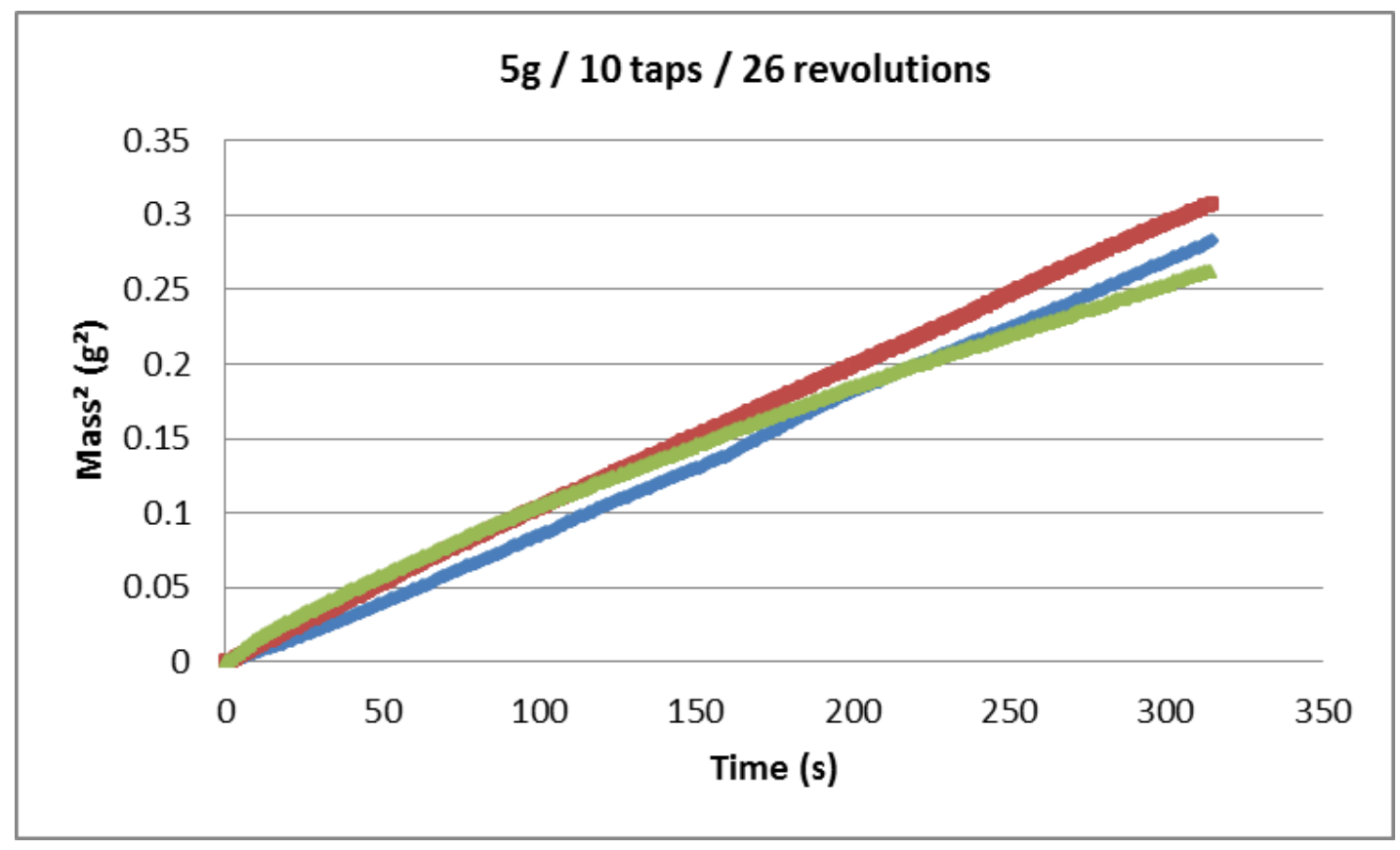

Figure 32 - Washburn response to sample preparation taps and number of revolutions

This sample preparation method compacted the powder inside the holder such that it reproduced the packing factor in the worst case scenario of a highly compacted agglomerate, where the particles fully touch each other, and the voids are as small 
as possible. Highly compact agglomerates are frequently found at the end of an inefficient dispersion process (i.e., those that can be crushed by fingers).

$\mathrm{N}$-hexane is used as a reference liquid in this methodology. It has a low surface energy and wets completely the voids inside the holder. The $n$-hexane mass adsorbed divided by its density is equal to the volume adsorbed, and when the sample is fully wetted, this volume is equal to the void volume of the agglomerates and is considered the maximum adsorbed liquid volume.

\subsubsection{Ring tensiometer}

The liquid surface tension was measured using the Du Noüy ring method. The method involves slowly lifting a ring made of platinum from the surface of a liquid. The force required to raise the ring from the liquid surface is measured and then related to the liquid surface tension (du Noüy, 1925).

The equipment used is a LAUDA model TD1, which is composed of an electronic unit where calibration and surface tension readings are performed and a mechanical unit where the sample and the ring is placed. Before using the equipment, the calibration is performed using a standard weight of $500 \mathrm{mg}$.

\subsubsection{Viscosity}

The methodology used to characterize the viscosity of the liquid solutions according to ASTM testing methods was the Ubbelohde capillary viscosimeter (Figure 33), which was chosen for its ability to measure low viscosity liquids and because it uses the principles of capillarity, which aligns with the studies herein.

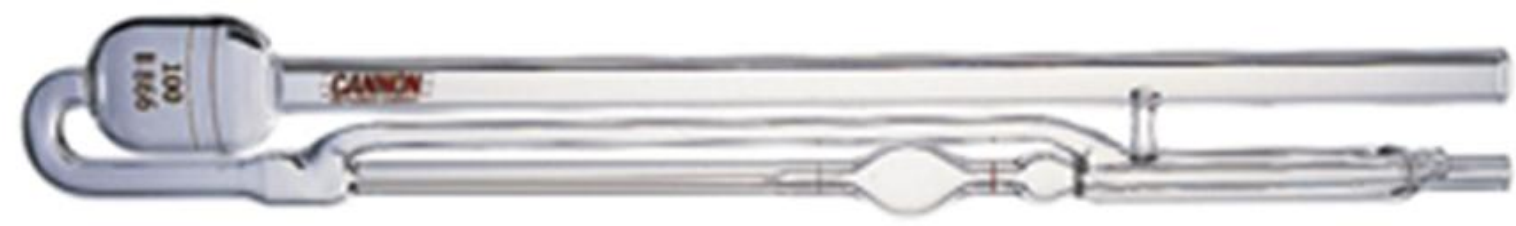

Figure 33 - Ubbelohde Viscosimeter (Cole-Parmer website).

A liquid is introduced into the reservoir and then sucked through the capillary and measuring bulb. The liquid is allowed to travel back through the measuring bulb, and the time it takes for the liquid to pass through two calibrated marks is the measure 
for viscosity. The Ubbelohde device has a third arm extending from the end of the capillary, which is open to the atmosphere. Hence, the pressure head depends on a fixed height, and no longer on the total volume of liquid.

\subsection{Design of experiments}

All experiments were run following a pre-established experimental design. Adjustments were made as required, the design of experiments (DOE) factors and the nominal levels (nominal because they might suffer adjustments as needed) are shown below.

Table 10 - Factors to determine the design of experiments

\begin{tabular}{ccc}
\hline Factor & Number of levels & Nominal Levels \\
\hline TiO2 Size & 3 & A, A', A" \\
TiO2 Surface & 3 & A, B, C \\
Liquid & 3 & n-Hexane, water, ethanol \\
Water surface tension & 3 & $72 ; 50 ; 45$ \\
\hline
\end{tabular}




\section{Results and Discussion}

\subsection{Liquid surface tension characterization}

Industrially available $\mathrm{TiO}_{2}$ suspensions, also called slurries, may contain from 60 to $75 \%$ solids (mass base), and the amount of dispersant relative to the powder can reach $3 \%$ in relation to the solids content, giving a maximum concentration of $2.25 \%$ of total dispersion. The dispersant is also used as a surfactant, affecting the liquid surface tension of the water in this study. Up to the limit of $3 \%$, the dispersant effect on the liquid surface tension has good reproducibility, as shown in Table 11 and Figure 34 , with data collected in this study.

Table 11 - Surface tension as a function of dispersant reproducibility analysis

\begin{tabular}{cccc}
\hline Dispersant Theoretical Concentration & $0 \%$ & $1.5 \%$ & $3.0 \%$ \\
\hline $1^{\text {st }}$ Run & 75.5 & 52.6 & 46.9 \\
$2^{\text {nd }}$ Run & 75.8 & 54.2 & 46.4 \\
$3^{\text {rd }}$ Run & 75.4 & 52.4 & 47.5 \\
Median & 75.6 & 53.1 & 46.9 \\
Std Deviation & 0.2 & 0.99 & 0.6 \\
\hline
\end{tabular}

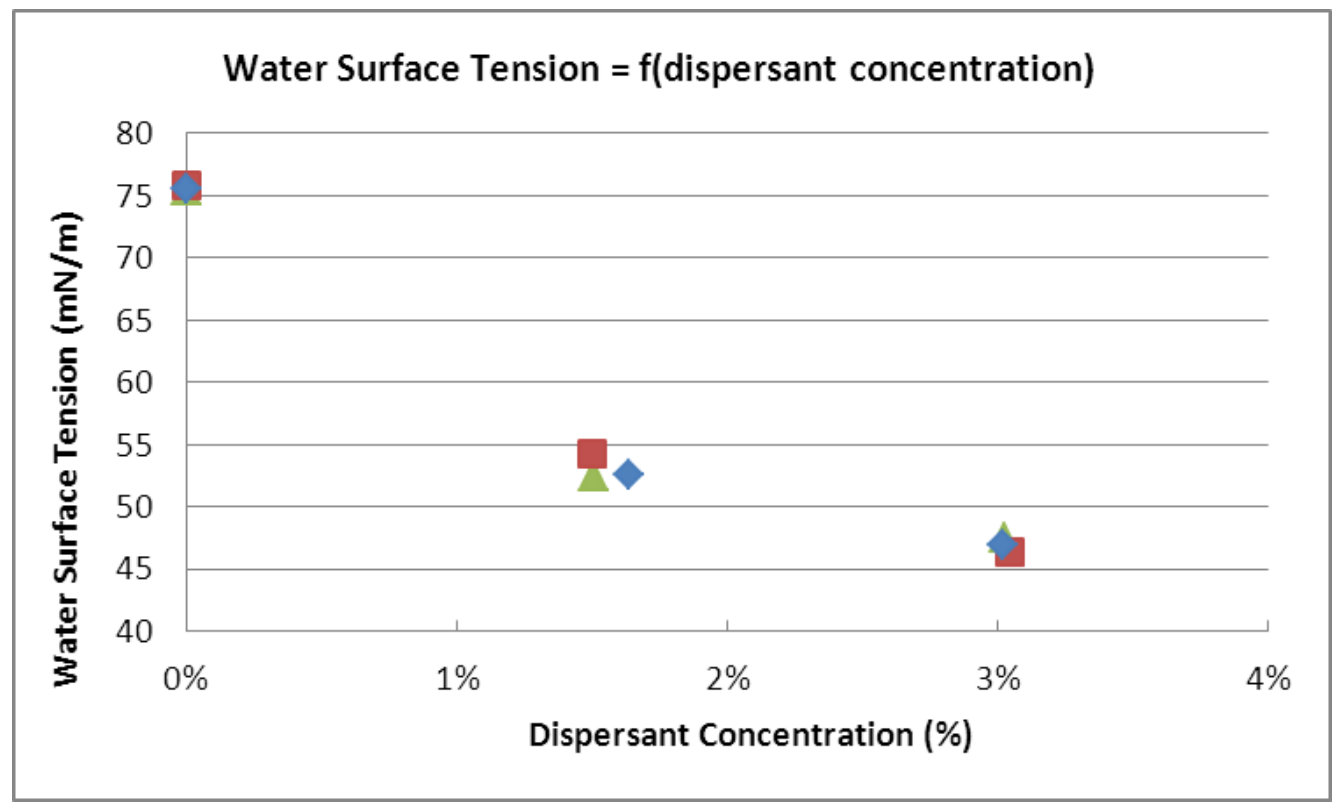

Figure 34 - Water surface tension as a function of dispersant concentration.

The surface tension values presented in Table 11 and Figure 34 were measured in the laboratory under the specific equipment conditions. They are adequate to study the test and equipment variability. However, in order to have an accurate value of the 
surface tension and apply it to the equations presented here, a correction factor must be put in place.

The correction factor is given for each equipment set and takes into account the ring geometry and density of the equipment used. In the present study the correction factor is obtained from Equation 29, which takes into account the liquid density, where STerr is the surface tension as measured, and $\rho_{\mathrm{l}}$ is the liquid density. The corrected value is a simple multiplication of the measured surface tension by the correction factor.

$f=0.8759+0.0009188 \cdot \frac{S \mathrm{Ter} r}{\rho_{\mathrm{i}}}$

Equation 29

The corrected value of the water surface tension as a function of dispersant concentration is shown in Figure 35, in which more points are shown, and it is possible to identify a significant drop in surface tension up to $1 \%$ dispersant concentration. After $1 \%$ concentration, the tendency is attenuated.

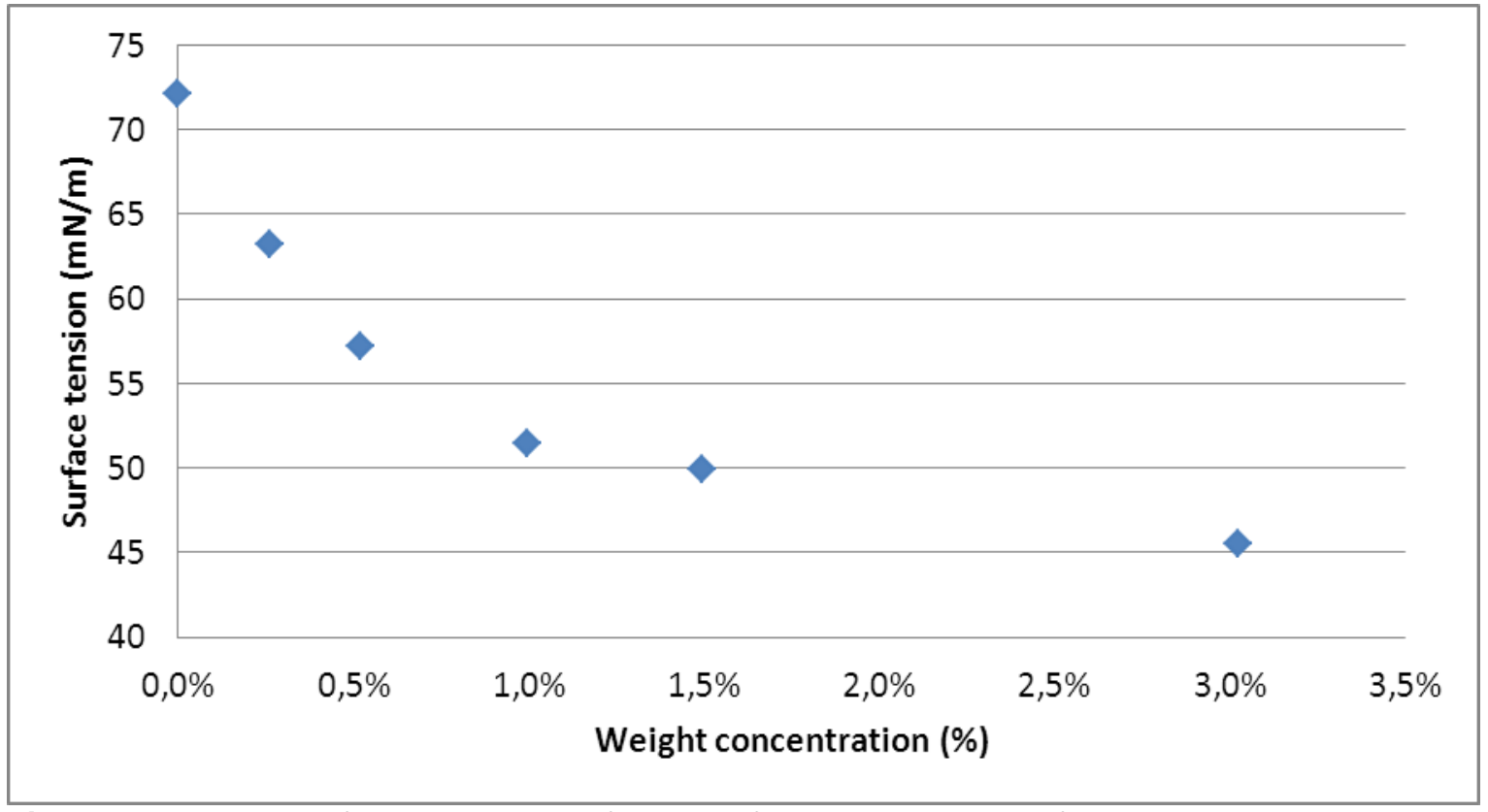

Figure 35 - Water surface tension as a function of the concentration of dispersant 


\subsection{Viscosity}

The addition of dispersant to water not only changes the water surface tension but also affects its viscosity, which can amount to a $25 \%$ difference when compared to water alone, as depicted in Figure 36.

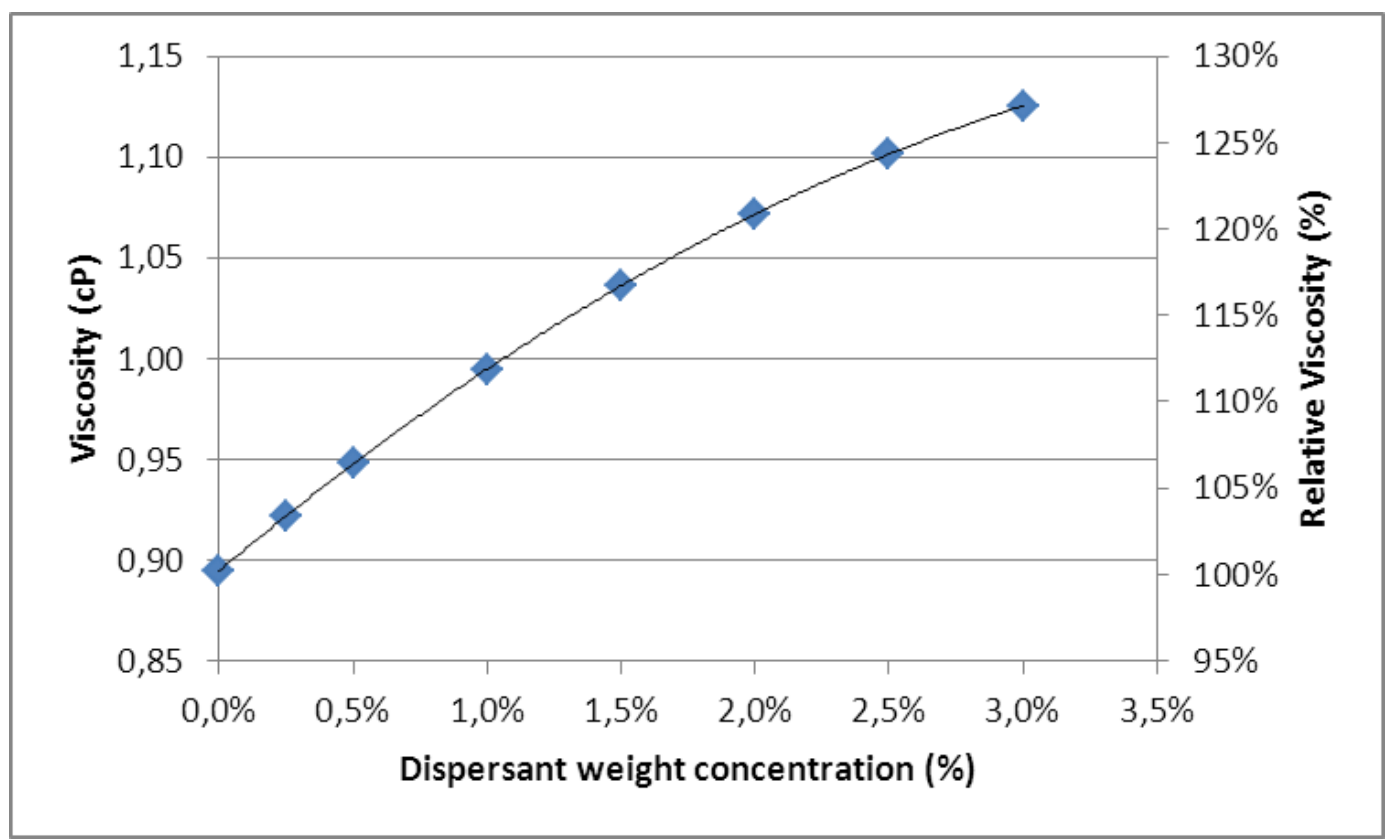

Figure 36 - Water - surfactant solution viscosity (left axis absolute, right axis relative) as a function of the concentration of surfactant.

The observed increase in viscosity imposes resistance to the liquid penetration into the pores of the agglomerates, which tends to reduce the wetting speed $(\mathrm{V} / \mathrm{t})$ as given in Equation 5. In this equation, if the capillary pressure is increased, the penetration speed also increases. Because the capillary pressure is a function of the liquid surface tension, the higher the surface tension, the higher the capillary pressure, which predicts an increase of the penetration speed if the liquid surface tension is increased.

$\frac{v}{t}=p \cdot\left(\frac{\pi r^{3}}{8 \eta L}\right)$

Equation 5

It seems contradictory that the higher the surface tension is, the higher the penetration speed. Therefore, in order to understand the penetration speed better, the adsorption rate must be studied. 


\subsection{Surface area}

Table 12 shows results from the measurement of the surface area of the samples used in this study. The surface area of samples A, A', and A" is approximately constant, although the particles have significantly different size distributions. This indicates that samples with larger particles may consist of aggregates, possibly formed in the wet treatment step of the production process. The void space in these aggregates play an important role in the wetting process.

Sample C was submitted to different kinds of thermal pre-treatment with the objective of verifying whether the organic hydrophilic treatment that this sample received could change if any adsorbed water was removed or reduced. It is possible to observe a small difference between the adsorption heat for the sample prepared using by the regular thermal treatment $\left(200{ }^{\circ} \mathrm{C} / 3 \mathrm{~h}\right)$ and a softer $\left(110^{\circ} \mathrm{C} / 3 \mathrm{~h}\right)$ treatment. The "C" constant for this sample (Equations 20 to 22) did not show any significant difference, except for the longer treatment $\left(110^{\circ} \mathrm{C} / 19,5 \mathrm{~h}\right)$, which caused a considerable reduction in this parameter.

Since the logarithm of "c" constant is proportional to the surface free energy which is dimensionally and numerically equivalent to the surface tension of the solid, the variation in the "c" constant denotes a potential variation in the surface tension of the solid (Salvador et al. 2002). The "c" constant for this sample (Equations 20 to 22) did not show any significant difference, except for the longer treatment $\left(110^{\circ} \mathrm{C} / 19,5 \mathrm{~h}\right)$, which caused a considerable reduction in this parameter.

Table 12 - Results of BET analysis of the samples used in this study

\begin{tabular}{cccccc}
\hline Grade & $\begin{array}{c}\text { Surface area } \\
\left(\mathbf{m}^{2} / \mathbf{g}\right)\end{array}$ & $\begin{array}{c}\text { “C” } \\
\text { constant }\end{array}$ & $\mathbf{r}^{\mathbf{2}}$ & Thermal treatment & $\begin{array}{c}\text { Adsorption } \\
\text { heat (kJ/mol) }\end{array}$ \\
A & 11.3 & 43.2 & 0.9996 & $200^{\circ} \mathrm{C} / 3 \mathrm{~h}$ & -3.16 \\
A $^{\prime}$ & 10.4 & 45.5 & 0.9998 & $200^{\circ} \mathrm{C} / 3 \mathrm{~h}$ & -3.13 \\
$\mathrm{~A}^{\prime \prime}$ & 11.2 & 41.3 & 0.9988 & $200^{\circ} \mathrm{C} / 3 \mathrm{~h}$ & -3.19 \\
$\mathrm{C}$ & 22.0 & 32.1 & 0.9989 & $200^{\circ} \mathrm{C} / 3 \mathrm{~h}$ & -3.35 \\
$\mathrm{C}$ & 20.9 & 31.6 & 0.9950 & $110^{\circ} \mathrm{C} / \mathrm{Bh}$ & -3.36 \\
$\mathrm{C}$ & 23.0 & 21.7 & 0.9940 & $110^{\circ} \mathrm{C} / 19.5 \mathrm{~h}$ & -3.60 \\
$\mathrm{~B}$ & 19.2 & 49.6 & 0.9998 & $200^{\circ} \mathrm{C} / 3 \mathrm{~h}$ & -3.07 \\
\hline
\end{tabular}

When sample $C$ was submitted to a more intense treatment $\left(110{ }^{\circ} \mathrm{C} / 19.5 \mathrm{~h}\right)$, the adsorption heat of nitrogen was significantly different. The "c" constant was reduced 
to below the minimum recommended value $(>30)$. The values of the coefficient of determination obtained by the linearization of the first 7 data points were also a screening factor, and for the alternative treatments of sample $C, r^{2}$ was much lower than the 0.999 as required by the method.

The differences in the results of Table 12 may be caused by water adsorbed from the environment. The typical humidity value of the dry powder is $0.5 \%$, which was removed by varying degrees according to the different pre-treatments.

Another interesting observation is that the adsorption heat can also be used to characterize the particle surface. The particles with similar surface properties have approximate values of adsorption heat, as for example samples $A, A^{\prime}$ and $A^{\prime \prime}$, with adsorption heat values of $3.16,3.13$ and $3.19 \mathrm{~kJ} / \mathrm{mol}$ respectively. It also shows that changes in the decimal can be significant as when comparing samples $A, B$ and $C$ with heating values of $3.16,3.35$ and $3.60 \mathrm{~kJ} / \mathrm{mol}$ respectively.

\subsection{Wetting speed}

According to the literature on the capillary rise method (Washburn) (Heertjes and Kossen, 1967; Kiesvaara and Yliruusib, 1993; Washburn, 1921), the maximum liquid adsorption by the packed powder is determined by the pore diameter $\left(\mathrm{C}_{\mathrm{w}}\right)$, liquid density and viscosity ( $\rho$ and $\eta$ ), time (t), liquid surface tension $(\gamma)$, and the liquid-solid surface tension interaction, which is described by Equation 11 .

$$
m^{2}=\left(C_{w} \rho^{2} \gamma \eta^{-1} \cos \theta\right) t
$$

Equation 11

where $C_{w}$ is the packing factor, $\rho$ is the liquid density, $\eta$ is the viscosity, $\gamma$ is the liquid surface tension, $t$ is the time of analysis, and $\theta$ is the contact angle between the liquid and solid. Assuming a constant packing factor $\left(\mathrm{C}_{\mathrm{w}}\right)$, and using the liquid density and viscosity ( $\rho$ and $\eta$ ) of each liquid, as $t \rightarrow \infty$, equilibrium between solid and liquid is achieved, and the mass of the adsorbed liquid is proportional to the wettability of the agglomerates.

The data obtained by the Washburn method are partially presented in Table 13 and fully presented in Appendix 1. 
The maximum volume of $n$-hexane adsorbed by the packed powder can be approximated as the pendular volume, previously described in equations 14 and 15, since it fills entirely the voids and is used to determine the pores volume and packing factor $\left(\mathrm{C}_{\mathrm{w}}\right)$.

$\sigma_{i \operatorname{mix}}=3 \cdot \frac{1-E}{\varepsilon} \cdot \frac{k}{\pi^{2}} \cdot \frac{1}{r^{7}} \cdot \eta L \cdot \frac{V_{E}^{2}}{!}$

Equation 30

$i=\frac{V_{p}^{2}}{\sigma_{i \pi \omega x}} \cdot \frac{1-k}{\underline{r}} \cdot \frac{k}{\pi^{2}} \cdot \frac{1}{r^{7}} \cdot 3 \pi L$

Equation 31

Proportionally the particles with higher volume of $n$-hexane require more energy to have its agglomerates broken.

Table 13 - Wetting speed results for the samples used in this study, based on the Washburn method.

\begin{tabular}{|c|c|c|c|}
\hline $\begin{array}{l}\text { Liquid surface } \\
\text { tension }(\mathrm{mN} / \mathrm{m}) \\
\text { and grade }\end{array}$ & $\begin{array}{l}\text { Adsorbed } \\
\text { Volume } \\
\text { (mL) }\end{array}$ & $\begin{array}{l}\text { Adsorption } \\
\text { Speed } \\
\left(g^{2 / s}\right)\end{array}$ & $\begin{array}{l}\text { Adsorption } \\
\text { Speed } \\
\left(\mathrm{mL}^{2} / \mathrm{s}\right)\end{array}$ \\
\hline \multicolumn{4}{|l|}{18} \\
\hline A & 3.290 & $4.68 \times 10^{-2}$ & $1.09 \times 10^{-1}$ \\
\hline$A^{\prime}$ & 3.222 & $5.08 \times 10^{-2}$ & $1.18 \times 10^{-1}$ \\
\hline A" & 2.419 & $2.19 \times 10^{-2}$ & $5.11 \times 10^{-2}$ \\
\hline B & 2.282 & $1.24 \times 10^{-2}$ & $2.88 \times 10^{-2}$ \\
\hline C & 2.069 & $8.24 \times 10^{-3}$ & $1.92 \times 10^{-2}$ \\
\hline \multicolumn{4}{|l|}{51} \\
\hline A & 0.319 & $3.40 \times 10^{-4}$ & $3.40 \times 10^{-4}$ \\
\hline$A^{\prime}$ & 0.265 & $2.35 \times 10^{-4}$ & $2.35 \times 10^{-4}$ \\
\hline$A^{\prime \prime}$ & 1.281 & $5.47 \times 10^{-3}$ & $5.47 \times 10^{-3}$ \\
\hline B & 0.419 & $6.04 \times 10^{-4}$ & $6.04 \times 10^{-4}$ \\
\hline $\mathrm{C}$ & 0.393 & $5.15 \times 10^{-4}$ & $5.15 \times 10^{-4}$ \\
\hline \multicolumn{4}{|l|}{73} \\
\hline A & 0.478 & $7.78 \times 10^{-4}$ & $7.78 \times 10^{-4}$ \\
\hline$A^{\prime}$ & 0.193 & $1.25 \times 10^{-4}$ & $1.25 \times 10^{-4}$ \\
\hline$A^{\prime \prime}$ & 1.238 & $5.15 \times 10^{-3}$ & $5.15 \times 10^{-3}$ \\
\hline B & 0.577 & $1.11 \times 10^{-3}$ & $1.11 \times 10^{-3}$ \\
\hline C & 0.529 & $9.37 \times 10^{-4}$ & $9.37 \times 10^{-4}$ \\
\hline
\end{tabular}


A sense of how the adsorbed mass behaves according to different values of the liquid surface tension is shown in Figure 37. In this figure, grade A" is used as an example to illustrate the changes in adsorption as a function of the liquid surface tension.

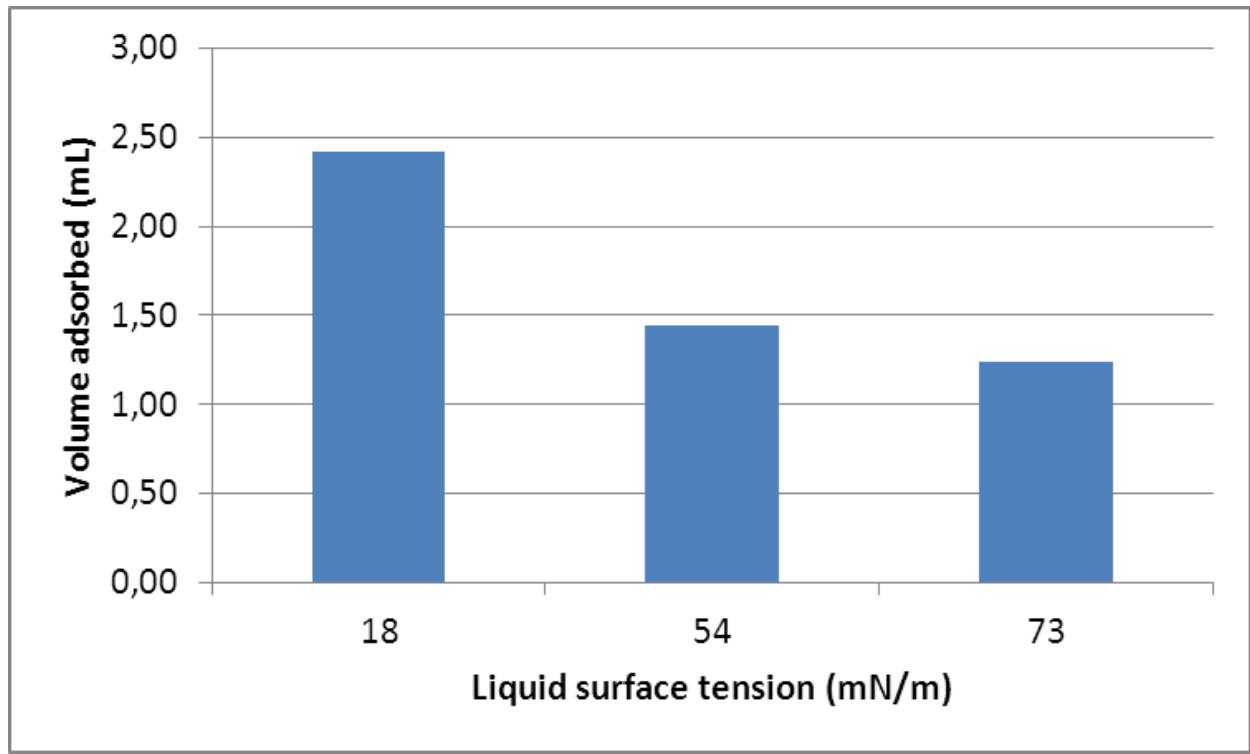

Figure 37 - Volume of liquid obtained at equilibrium point for sample A" for different values of the liquid surface tension

By using the data in Table 13, it is possible to calculate the contact angle between the liquid and solid $(\theta)$ in Equation 11. With this contact angle, the surface tension of the solid can be determined by Equation 32 (Patton, 1979).

$\gamma_{s}=\frac{(y-\theta / B)(\cos \theta+1)}{2}$

Equation 32

The contact angles between the particles and the liquids are calculated based on the data from Table 13 and are shown in Table 14. 
Table 14 - Calculated contact angle between particle and liquid.

\begin{tabular}{cccc}
\hline Grade & $\begin{array}{c}\text { Liquid surface } \\
\text { tension }(\mathbf{m N} / \mathbf{m})\end{array}$ & \multicolumn{2}{c}{ Contact angle } \\
\cline { 3 - 4 } A & 51 & 1.56 & Radian \\
& 73 & 1.55 & 89 \\
A $^{\prime}$ & 51 & 1.56 & 90 \\
& 73 & 1.57 & 90 \\
A" $^{\prime \prime}$ & 51 & 1.18 & 68 \\
& 73 & 1.26 & 72 \\
B & 51 & 1.50 & 86 \\
& 73 & 1.45 & 83 \\
C & 51 & 1.48 & 85 \\
& 73 & 1.42 & 82 \\
\hline
\end{tabular}

The calculated solid surface tension is shown in Table 15.

\begin{tabular}{ccc}
\multicolumn{3}{c}{$\begin{array}{l}\text { Table } 15 \text { - Calculated solid surface tension } \\
\text { for the samples used in this study }\end{array}$} \\
\hline Grade & $\begin{array}{c}\text { Solid surface } \\
\text { tension (mN/m) }\end{array}$ & $\begin{array}{c}\text { Shape } \\
\text { factor } \\
\text { (i) }\end{array}$ \\
\hline A & 29 & 4.6 \\
\hline A $^{\prime}$ & 29 & 4.4 \\
A" $^{\prime \prime}$ & 41 & 8.7 \\
B & 30 & 7.5 \\
C & 32 & 7.3 \\
\hline
\end{tabular}

It is important to highlight that although sample A" has the same surface treatment as $A$ and $A^{\prime}$, it presented a different surface tension than these samples. The explanation for this different behavior is the shape factor of sample A", which is almost double that of the other samples. The particle size distribution is another difference, compared to the other two samples, as shown in Figure 38. Instead of the narrow distribution observed for samples $A$ and $A$ ', sample A" shows a broad distribution throughout the sizes, reflecting the lack of micronization. 


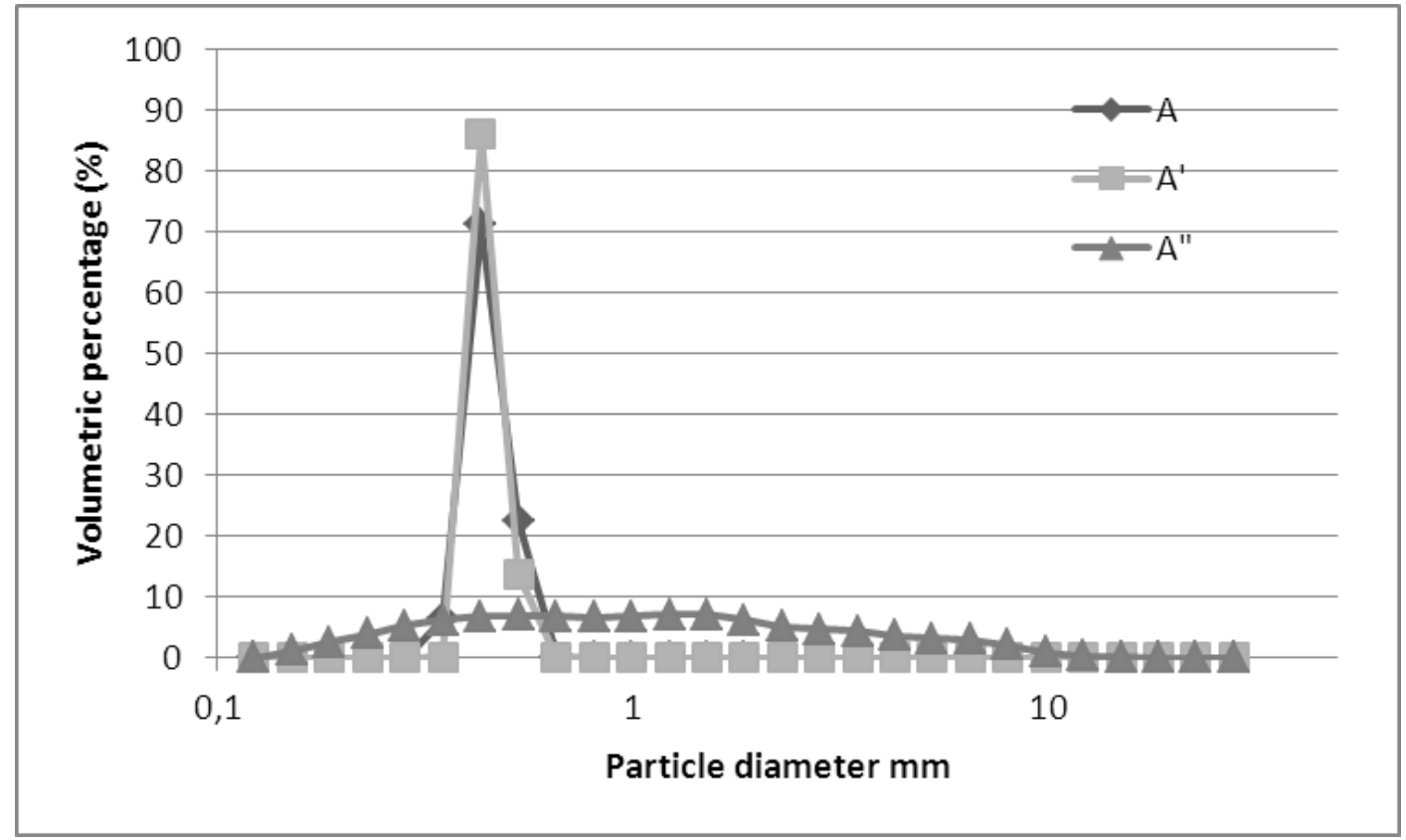

Figure 38 - Particle size distribution of samples A, A', and A".

Although the literature (Dang-Vu, Hupka, Drzymała, 2006) claims that particle roughness does not affect the contact angle of interaction between the liquid and solid, since the shape factor in this case is a function of the number of agglomerates, then the roughness of the particles may be similar. Hence, changes in the size and shape affect the packing factor of these particles compared to the other ones, as shown in Table 16.

Table 16 - Packing factor, $\mathrm{C}_{\mathrm{w}}$, for the samples used in this study

$\begin{array}{cc}\text { Grade } & \mathbf{C}_{\mathrm{w}}\left(\mathrm{m}^{5}\right) \\ \text { A } & 0.0321 \\ \text { A }^{\prime} & 0.0348 \\ \text { A" }^{\prime \prime} & 0.0150 \\ \text { B } & 0.0085 \\ \text { C } & 0.0056\end{array}$

As previously mentioned, $\mathrm{C}_{\mathrm{w}}$ is a function of the pore radii, which is a function of the packing factor. When the particle size distribution is wider, the particles accommodate better, which reduces the mean pore radius. When the particle size distribution and shape factor are too different, these factors may affect the particle surface tension estimation. Therefore, this study considers the surface tensions calculated for samples A and A', only. 
Figure 39 presents the volume of $n$-hexane adsorbed by the particles in the study. For samples $A$ and $A^{\prime}$, it is clear that an increment in the particle shape factor, $i$ (BET/Mom), defined in Equation 9, reflects a reduction in pore volume (and $n$ hexane adsorption). It is also clear that different particles (B, $C$ and $A$ ") can present similar i values as well as similar pore volumes. This behavior reinforces the fact that the increments in surface area are due to changes in shape rather than changes in roughness.

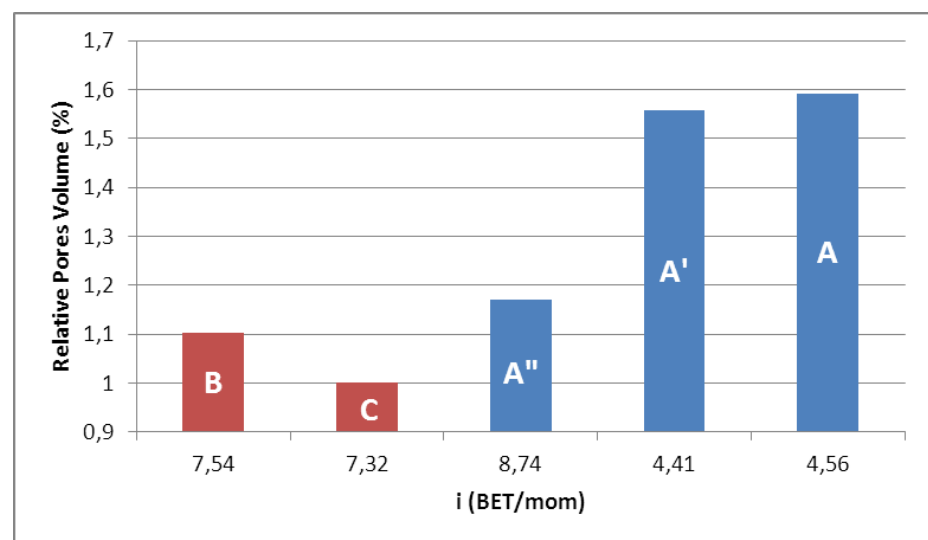

Figure 39 - Relative pore volume (\%) as a function of particle class and $\mathrm{i}$, for the samples tested in this study.

Equation 7 and Equation 5 are used to calculate the meniscus radius, the capillary radius, and the capillary pressure, which are shown in Table 17.

Table 17 - Capillary radius, capillary pressure, and the meniscus radius of the interaction between particle and liquid.Continues.

\begin{tabular}{cccc}
$\begin{array}{c}\text { Liquid surface } \\
\text { tension }(\mathbf{m N} / \mathbf{m})\end{array}$ & $\begin{array}{c}\text { Capillary } \\
\text { radius }(\boldsymbol{\mu m})\end{array}$ & $\begin{array}{c}\text { Meniscus } \\
\text { radius } \\
(\boldsymbol{\mu m})\end{array}$ & $\begin{array}{c}\text { Capillary } \\
\text { Pressure } \\
\left(\mathbf{m N} / \mathbf{m}^{2}\right)\end{array}$ \\
\hline $\mathbf{A}$ & $\mathbf{0 . 1 4 0}$ & $\mathbf{0 . 0 3 5}$ & $\mathbf{1 6 9 3}$ \\
\hline 58.9 & 0.145 & 0.036 & 1621 \\
54.5 & 0.178 & 0.045 & 1222 \\
50.9 & 0.136 & 0.034 & 1498 \\
45.5 & 0.135 & 0.034 & 1359 \\
73.0 & 0.106 & 0.026 & 2765 \\
$\mathbf{A}^{\prime}$ & $\mathbf{0 . 1 4 2}$ & $\mathbf{0 . 0 3 6}$ & $\mathbf{1 6 0 1}$ \\
\hline 58.9 & 0.125 & 0.031 & 1889 \\
54.5 & 0.147 & 0.037 & 1482 \\
50.9 & 0.149 & 0.037 & 1368 \\
45.5 & 0.143 & 0.036 & 1278 \\
73.0 & 0.147 & 0.037 & 1989 \\
\hline
\end{tabular}


Table 17 - Capillary radius, capillary pressure, and the meniscus radius of the interaction between particle and liquid.conclusion

\begin{tabular}{cccc}
$\begin{array}{c}\text { Liquid surface } \\
\text { tension }(\mathbf{m N} / \mathbf{m})\end{array}$ & $\begin{array}{c}\text { Capillary } \\
\text { radius }(\boldsymbol{\mu m})\end{array}$ & $\begin{array}{c}\text { Meniscus } \\
\text { radius } \\
(\mu \mathrm{m})\end{array}$ & $\begin{array}{c}\text { Capillary } \\
\text { Pressure } \\
\left(\mathbf{m N} / \mathbf{m}^{2}\right)\end{array}$ \\
\hline A $^{\prime \prime}$ & $\mathbf{0 . 0 6 4}$ & $\mathbf{0 . 0 1 6}$ & $\mathbf{3 6 2 5}$ \\
\hline 58.9 & 0.056 & 0.014 & 4226 \\
54.5 & 0.063 & 0.016 & 3485 \\
50.9 & 0.066 & 0.017 & 3065 \\
45.5 & 0.074 & 0.019 & 2455 \\
73.0 & 0.060 & 0.015 & 4893 \\
B & $\mathbf{0 . 0 7 8}$ & $\mathbf{0 . 0 2 0}$ & 3003 \\
58.9 & 0.075 & 0.019 & 3122 \\
54.5 & 0.079 & 0.020 & 2770 \\
50.9 & 0.080 & 0.020 & 2546 \\
45.5 & 0.092 & 0.023 & 1982 \\
73.0 & 0.064 & 0.016 & 4595 \\
C & 0.065 & 0.016 & 3544 \\
\hline 58.9 & 0.061 & 0.015 & 3872 \\
54.5 & 0.066 & 0.017 & 3285 \\
50.9 & 0.071 & 0.018 & 2863 \\
45.5 & 0.070 & 0.018 & 2589 \\
73.0 & 0.057 & 0.014 & 5111 \\
\hline & & &
\end{tabular}

The capillary radius and the meniscus radius can be related to the mean particle size, generating a dimensionless factor, as shown in Table 18. 
Table 18 - Dimensionless relations between capillary and particle radii and meniscus and particle radii

\begin{tabular}{|ccc|}
\hline A & $\begin{array}{c}\text { Capillary } \\
\text { particle radii } \\
\text { relation }\end{array}$ & $\begin{array}{c}\text { Meniscus/particle } \\
\text { radii relation }\end{array}$ \\
\hline 18 & 0.402 & 0.100 \\
51 & 0.618 & 0.154 \\
73 & 0.481 & 0.120 \\
A' $^{\prime}$ & & \\
\hline 18 & 0.358 & 0.090 \\
51 & 0.584 & 0.146 \\
73 & 0.576 & 0.144 \\
A" $^{\prime \prime}$ & & \\
\hline 51 & 0.219 & 0.055 \\
73 & 0.191 & 0.048 \\
B & 0.172 & 0.043 \\
\hline 18 & & \\
51 & 0.298 & 0.074 \\
73 & 0.374 & 0.093 \\
C & 0.296 & 0.074 \\
\hline 18 & 0.278 & 0.069 \\
51 & 0.343 & 0.086 \\
73 & 0.275 & 0.069 \\
\hline
\end{tabular}

Figure 40 shows the volume of adsorbed liquid for each particle in fluids with different surface tensions. As shown in the figure, the adsorbed volume is smaller for the bigger particles. Regarding the lower surface tensions, the ratio to particle size is understandable when the bigger particles have higher bulk density, which means there is less space between the particles. Therefore, a lower volume of liquid is adsorbed, which can be observed for $n$-hexane (liquid surface tension equal to 18 $\mathrm{mN} / \mathrm{m}$ ). Particles $A$ and $A^{\prime}$ adsorbed a larger liquid volume than the bigger particles, A", C and D.

The reduction in adsorbed volume as a function of the liquid surface tension, as shown in Figure 40, can be further explained by the meniscus radius formed by the interaction of the liquid and solid. Using particle class $A$ as an example (A, A', A" have the same surface treatment), it is possible to observe that even though a 
reduction in adsorbed volume occurred for particle A", it adsorbed four times more liquid than $A$ and $A^{\prime}$.

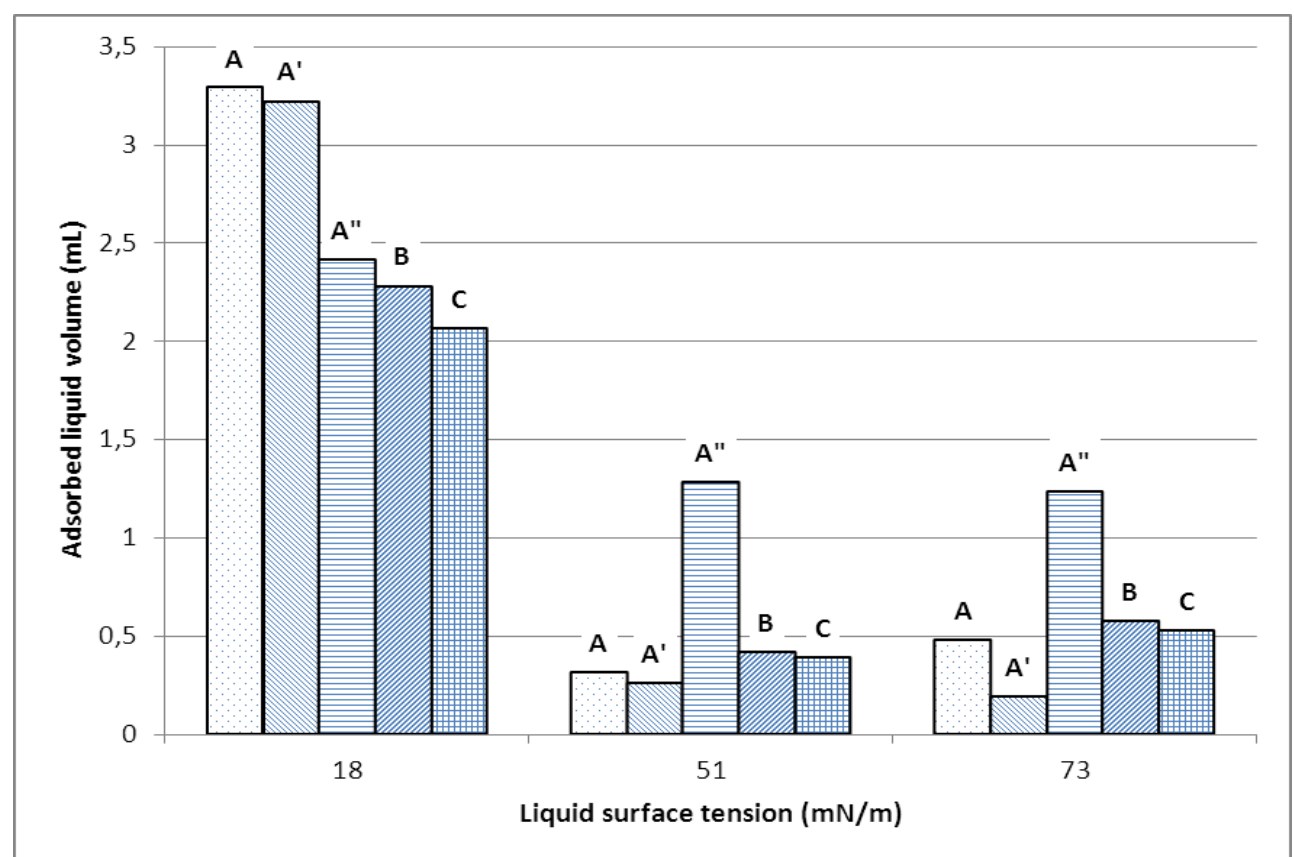

Figure 40 - Volume adsorbed by particles with different mean sizes and liquids with different values of surface tension.

Figure 41 shows the ratio between the meniscus radius and the mean particle radius $(\mathrm{D}(3,2) / 2)$ as a function of the liquid surface tension. The figure shows that for sample A" the meniscus radius did not overcome $6 \%$ of the mean particle size for any liquid. However, for samples $A$ and $A^{\prime}$, it reached between 12 to $15 \%$ of the mean particle size. 


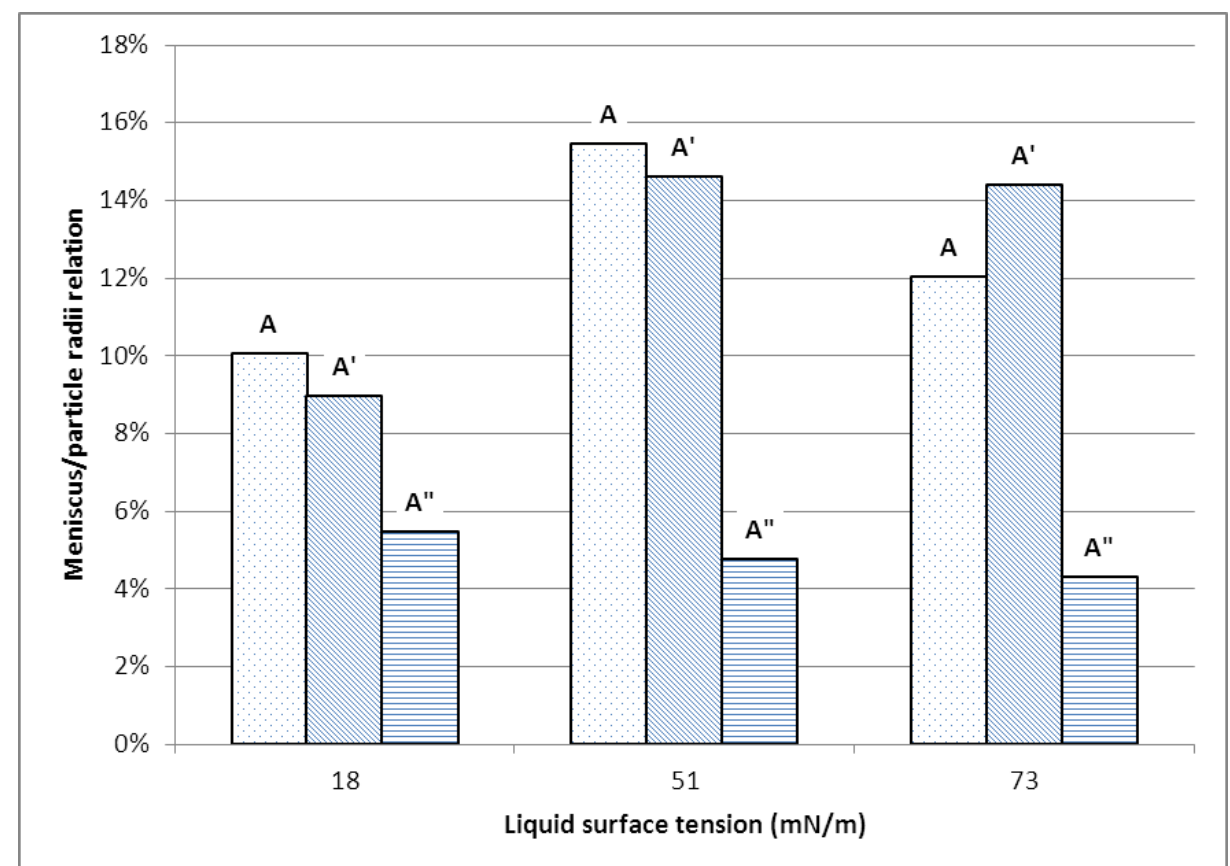

Figure 41 - The ratio of meniscus and particle radii as a function of liquid surface tension

Nonetheless, it is possible to conclude that there might exist a correlation between the ratio of meniscus radii to particle radii and the volume of the adsorbed liquid. This empirical relation is presented in Figure 42. A logarithmic correlation, obtained by least squares, explains almost $91 \%$ of the variation in the volume of adsorbed liquid ( $\mathrm{r}^{2}$ equal to 0.9088$)$, indicating a clear correlation.

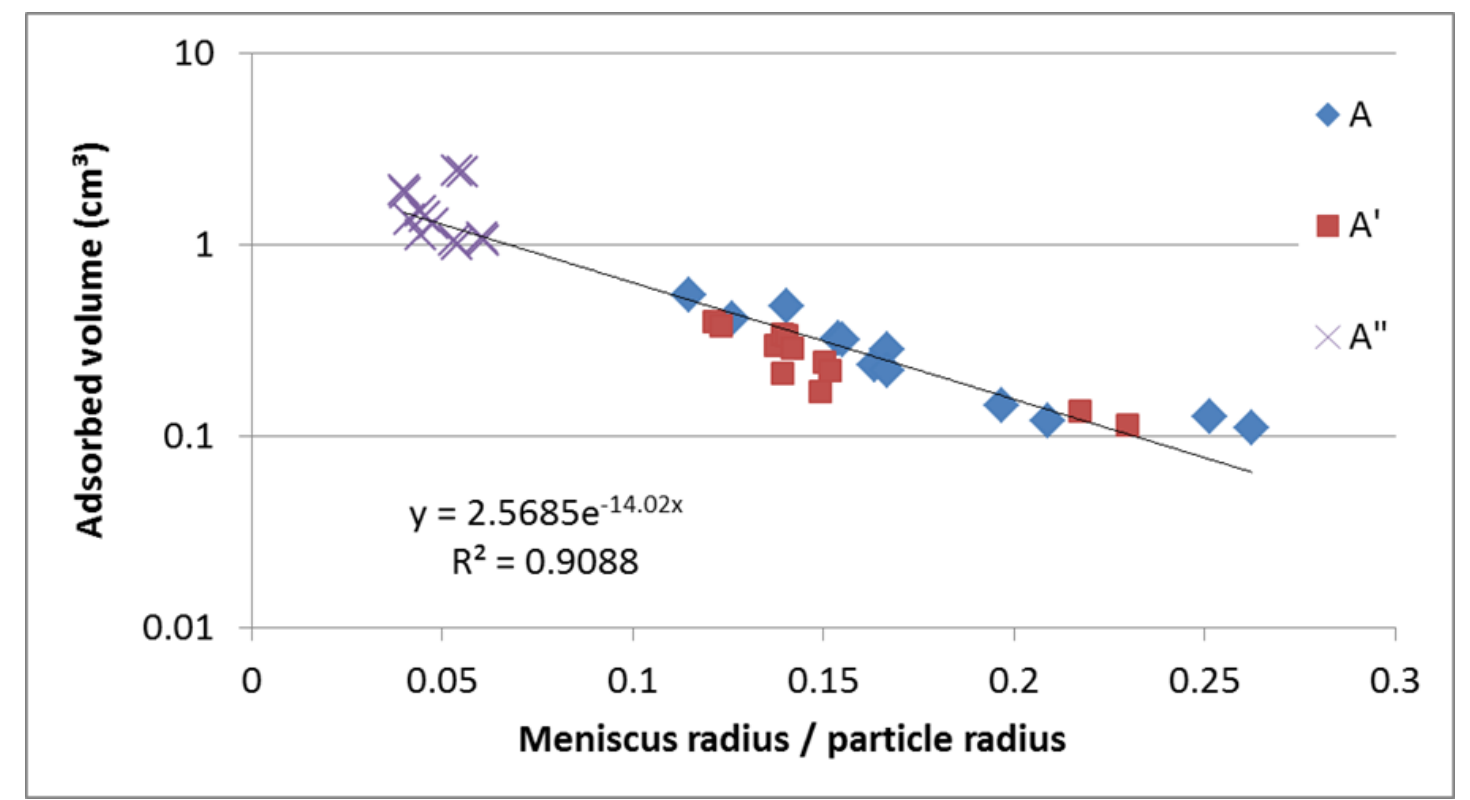

Figure 42 - Adsorbed liquid volume as a function of the ratio between meniscus radius and particle diameter for samples $A, A^{\prime}$, and A".

The wettability for several particle sizes can now be compared. Figure 43 , shows the result of including different particle sizes and particles with different surface 
treatments in the correlation. The correlation is less clear than that shown in Figure 42 , but the observed tendency remains $\left(r^{2}\right.$ equal to 0.75$)$.

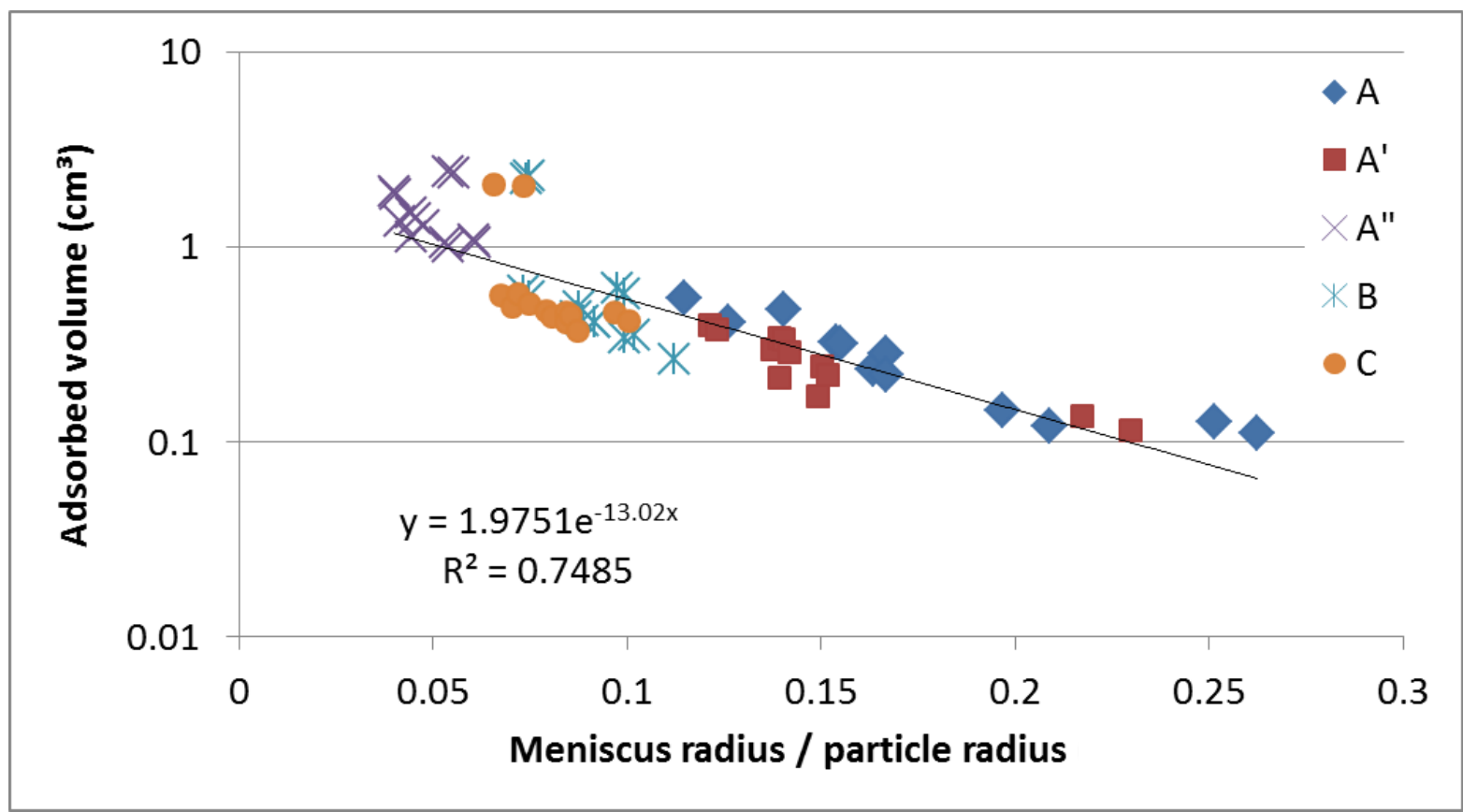

Figure 43 - Liquid volume adsorption as a function of the ratio between meniscus and particle radii for particles $A, A^{\prime}, A^{\prime \prime}, B$, and C.

Figure 44 presents the liquid adsorption speed $(\mathrm{mL} / \mathrm{s})$ obtained in the experiments as a function of the ratio between liquid and solid surface tensions $\left(\gamma_{i} / \gamma_{s}\right)$.

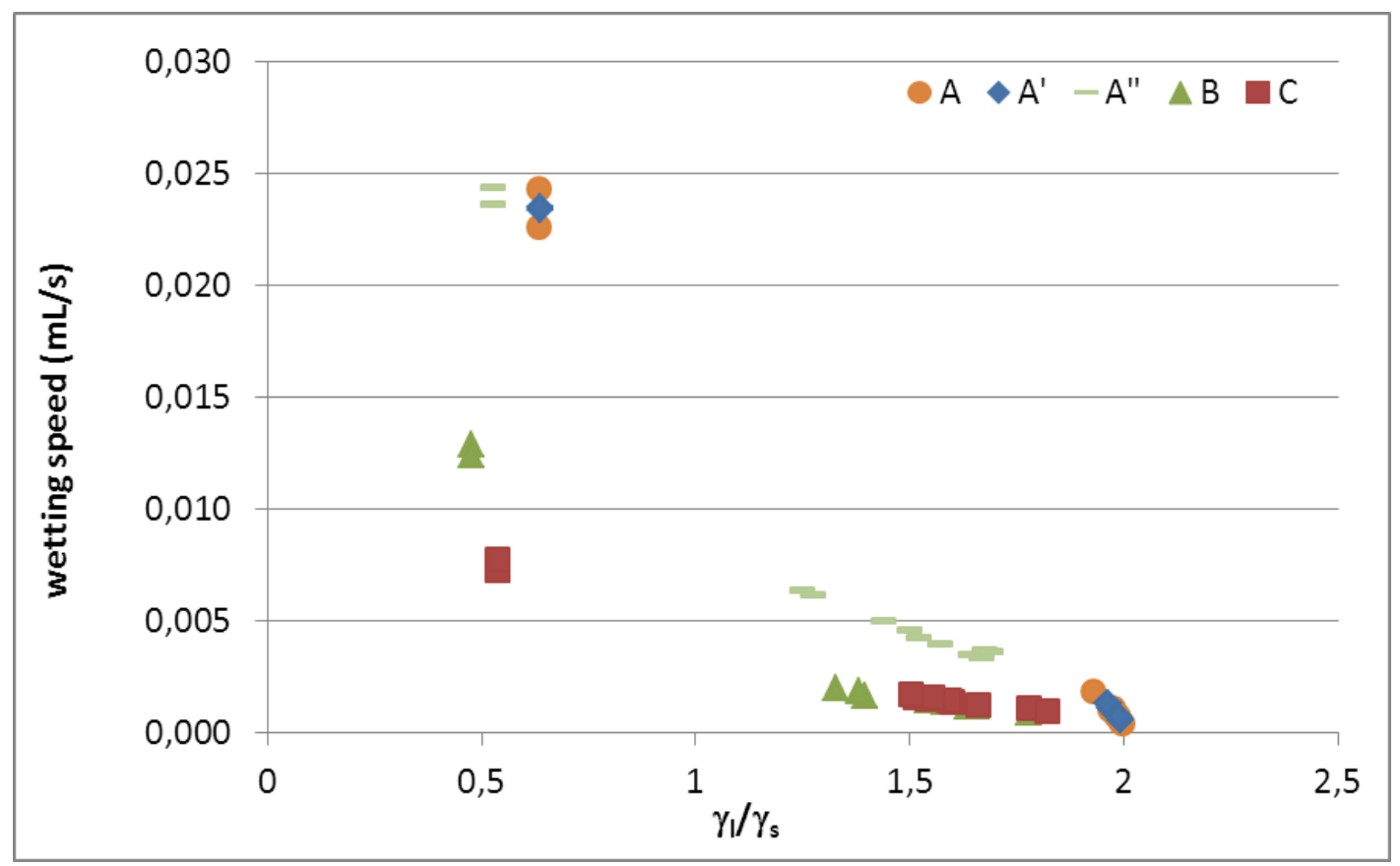

Figure 44 - Liquid volume adsorption speed as a function of the ratio between liquid and solid surface tensions for the samples used in this study. 
As shown in Figure 38, the maximum adsorbed mass is proportional to the liquid surface tension. However, as depicted in Figure 45, the liquid volume adsorption speed is clearly a function of the ratio between the liquid and solid surface tensions and the shape factor. In this case, the lower the liquid surface energy is in relation to the solid surface tension for any shape factor value (i), the greater the adsorbed volume speed.

As shown in Figure 45, when the particle shape factor is taken into account, it is clear that similar shape factors have similar behavior, independently from the surface treatment. For instance, for larger shape factor (i) values, i.e., when particles accommodate themselves reducing the void volume in the bulk of the powder, the absorbed $\mathrm{n}$-hexane volume is lower, as discussed in Chapter 2.2.1.

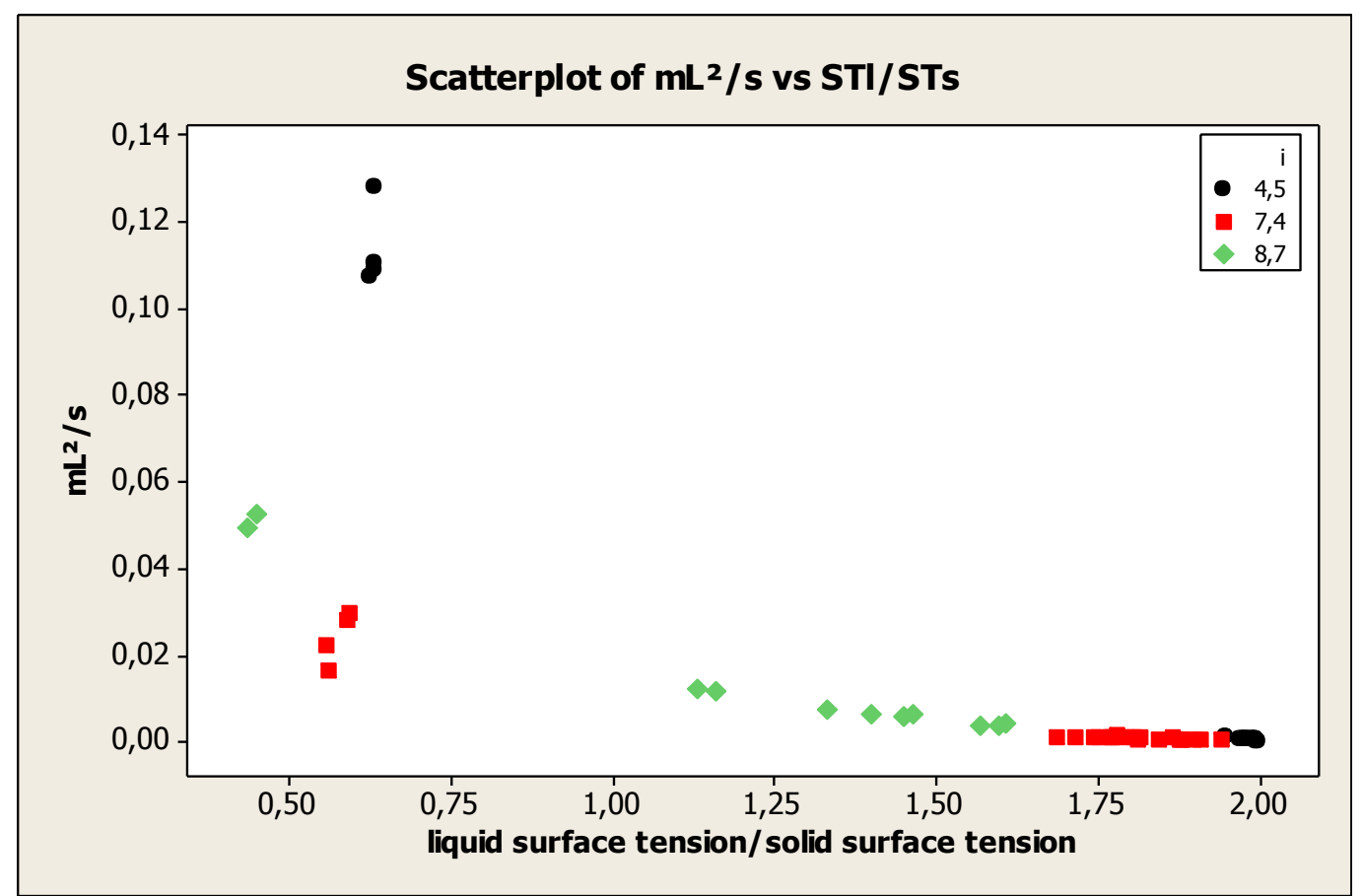

Figure 45 - Adsorption speed as a function of the ratio between liquid and solid surface tensions for different values of the shape factor $i$.

Figure 45 shows that particles with a surface roughness of 7.4 and 8.7 behave similarly. Figure 46 presents fitted line plots assuming a quadratic regression that shows the adsorption speed as a function of the surface interaction between the liquid and solids, expressed as $\gamma_{i} / \gamma_{s}$. The coefficient of determination of 0.877 indicates a good quality of the fitting. 


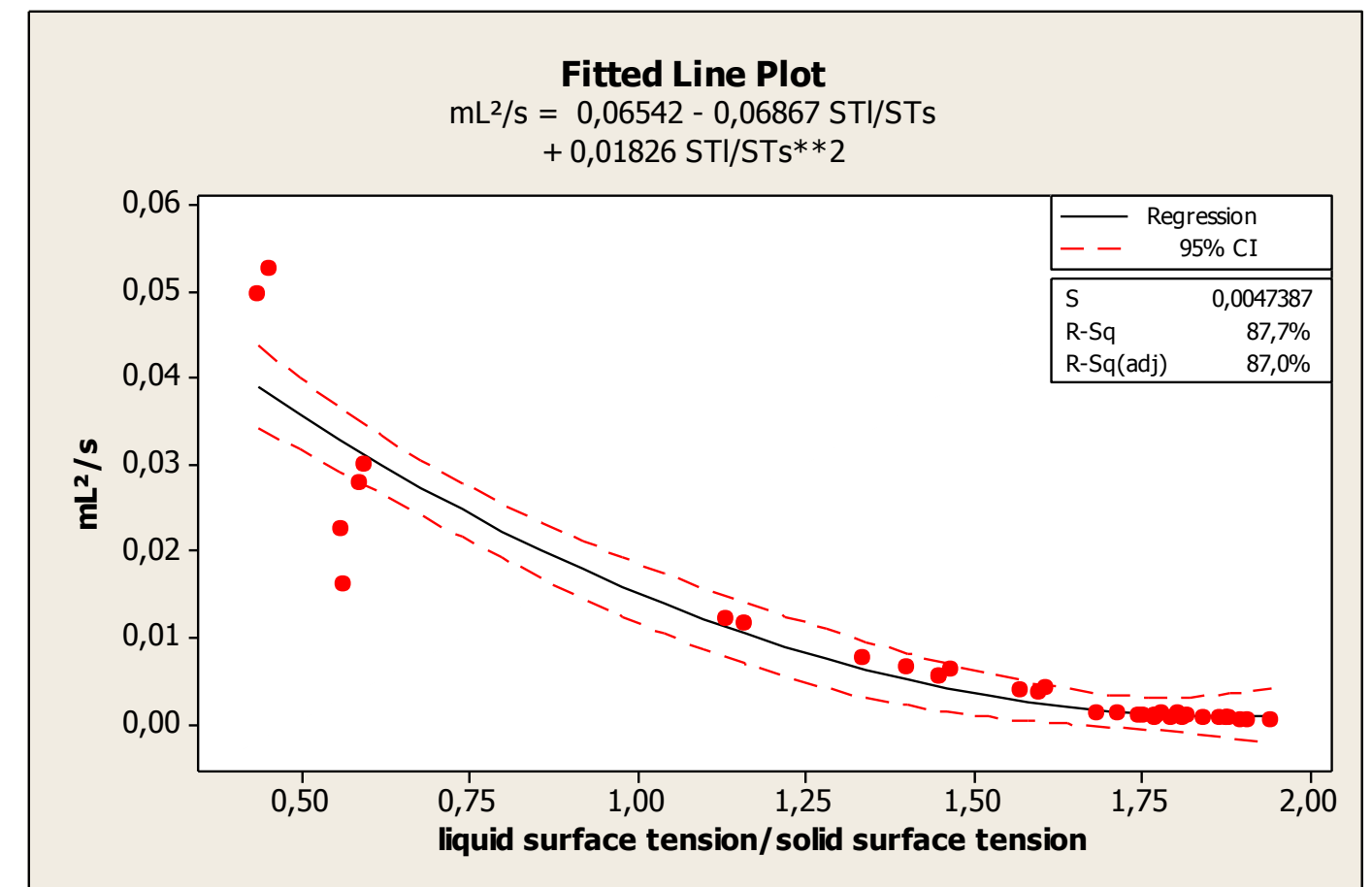

Figure 46 - Fitted line plot for liquid volume adsorption speed as a function of the ratio of liquid and solid surface tension, for $\mathrm{i}=7.4$ and 8.5 .

The same phenomena are observed in particles with shape factor (i) equal to 4.5 , as shown in Figure 47, for which a coefficient of determination equal to 0.997 was obtained for the fitting.

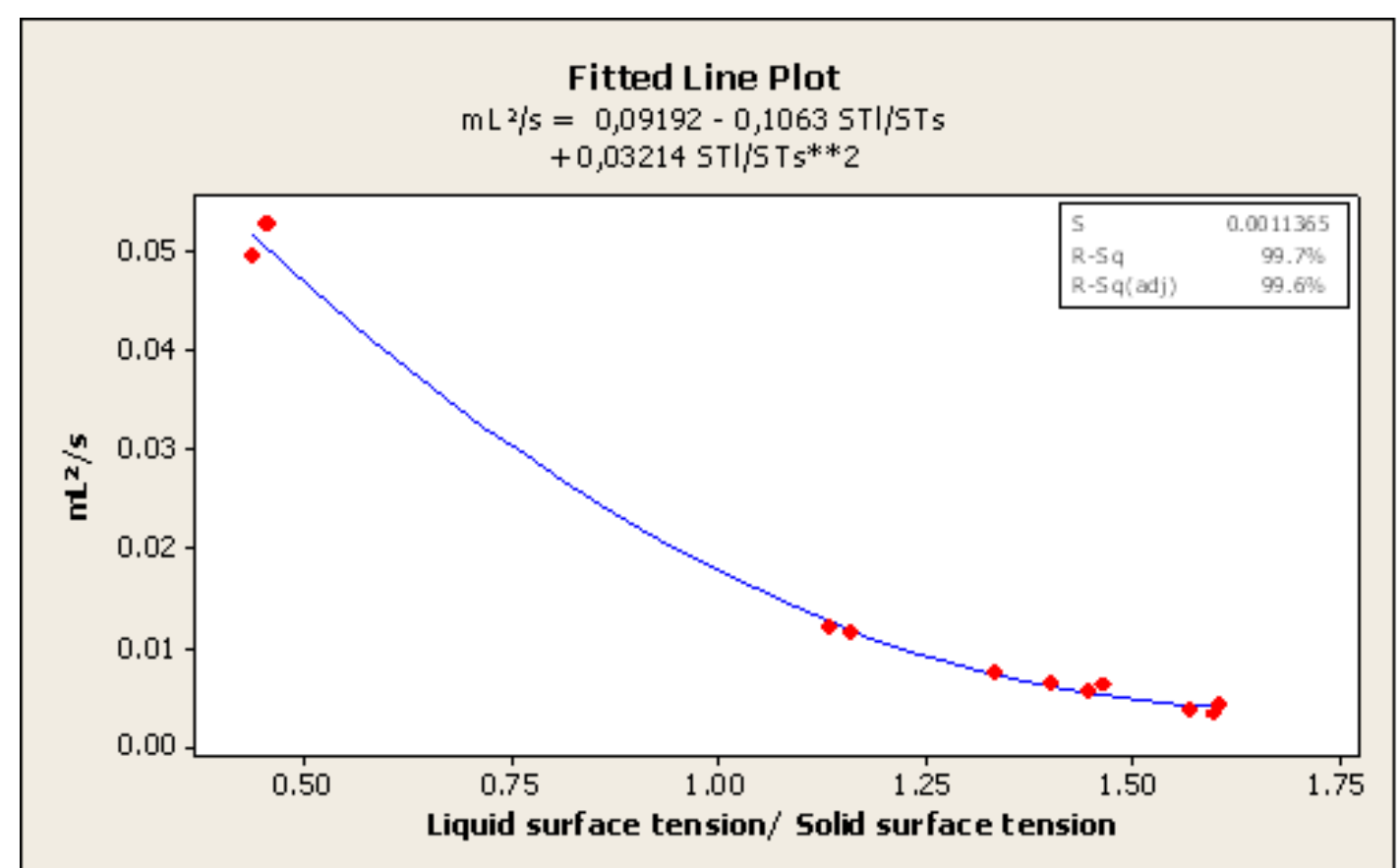

Figure 47 - Fitted line plot for liquid volume adsorption speed as a function of the liquid and solid surface tension rate $(i=4.5)$.

Based on the values of the liquid adsorption speed, liquid viscosity, and liquid surface tension, it is possible to calculate the meniscus radius. The ratio between the 
meniscus radius $\left(r_{m}\right)$ and particle radius increases as the ratio between the liquid and solid surface tension increases, as shown in Figure 48. For each liquid "family" the relationship is different. In the example for water, if the $\gamma_{2} / y_{s}$. ratio is equal to 2 , then the meniscus radius increases exponentially. For larger values of $\gamma_{2} / \gamma_{s}$, the liquid cannot penetrate the solid agglomerate, as illustrated in Figure 49, where a pellet of $\mathrm{TiO}_{2}$ powder is prepared, and then a drop of deionized water is placed on its surface. The left picture shows that the water remains on top of the pellet, whereas the drop of the water on the right is adsorbed. The left-side powder is designed to be incorporated into polyolefins and might have a surface tension around $18 \mathrm{mN} / \mathrm{m}$. In this case, the ratio between solid and liquid surfaces is well beyond 2 (about 4 in this case). In this case the meniscus radius is too large and the liquid does not wet the powder voluntarily.

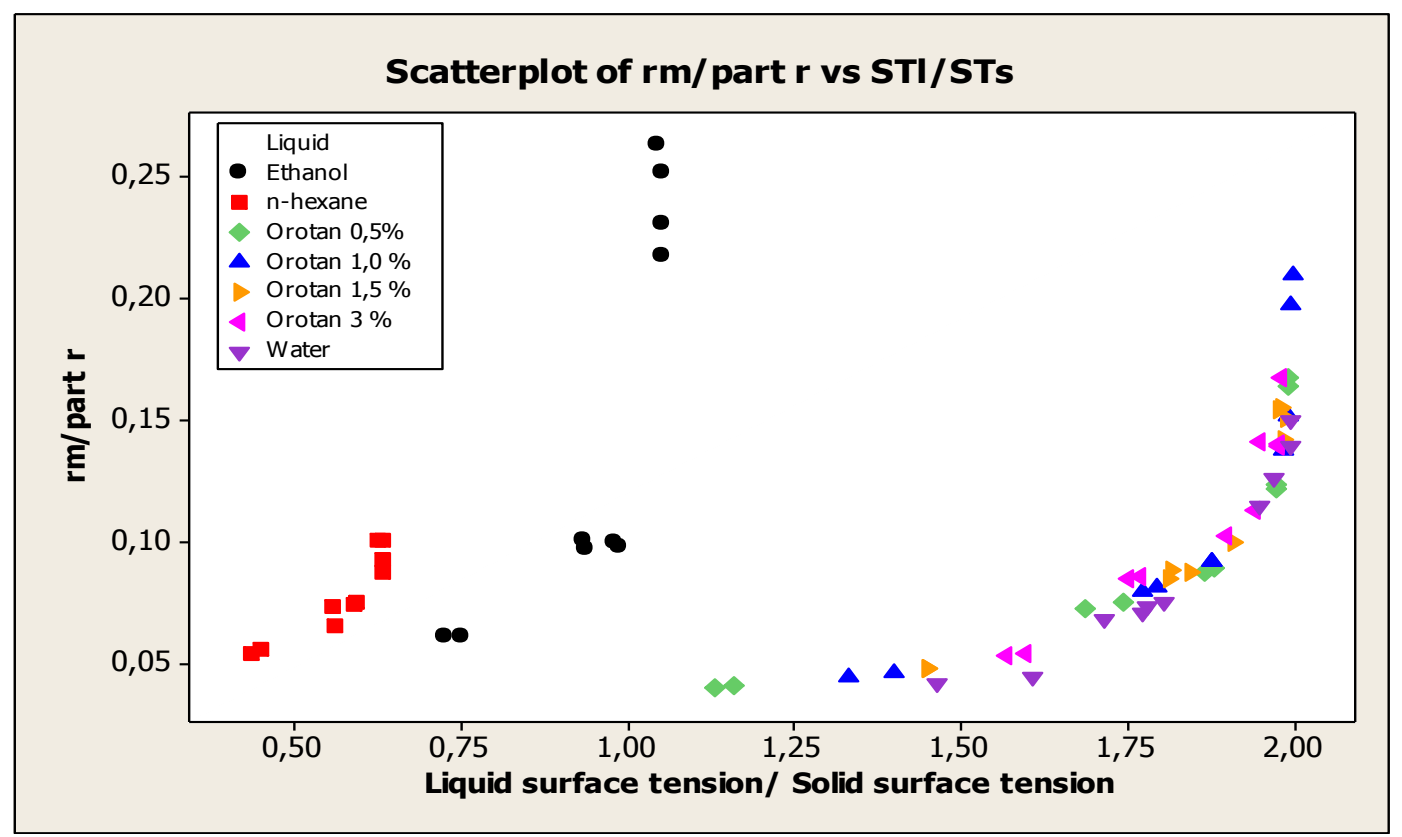

Figure 48 - Relationship of the ratio between meniscus and particle radius and the liquid to solid surface tension ratio, for all samples.

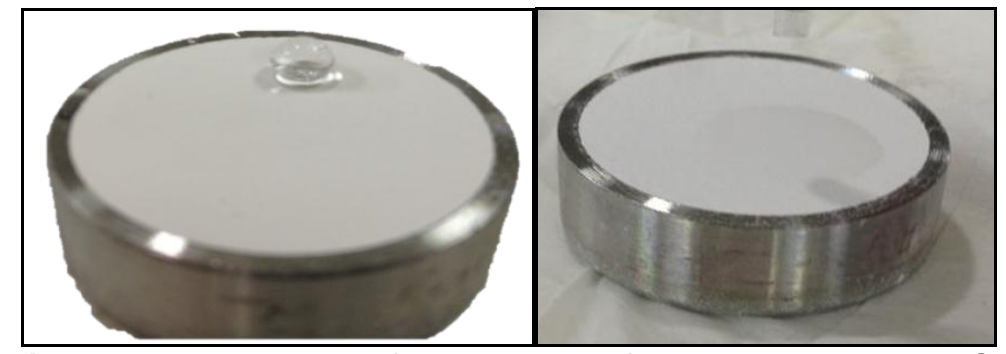

Figure 49 - Illustration of D.I. Water surface interaction with $\mathrm{TiO}_{2}$ pellets: left: $\mathrm{TiO}_{2}$ for polyolefines with hydrophobic surface treatment; right: coatings grade with hydrophilic surface treatment. 
The phenomena presented in figures 49 and 50 can be better explained by

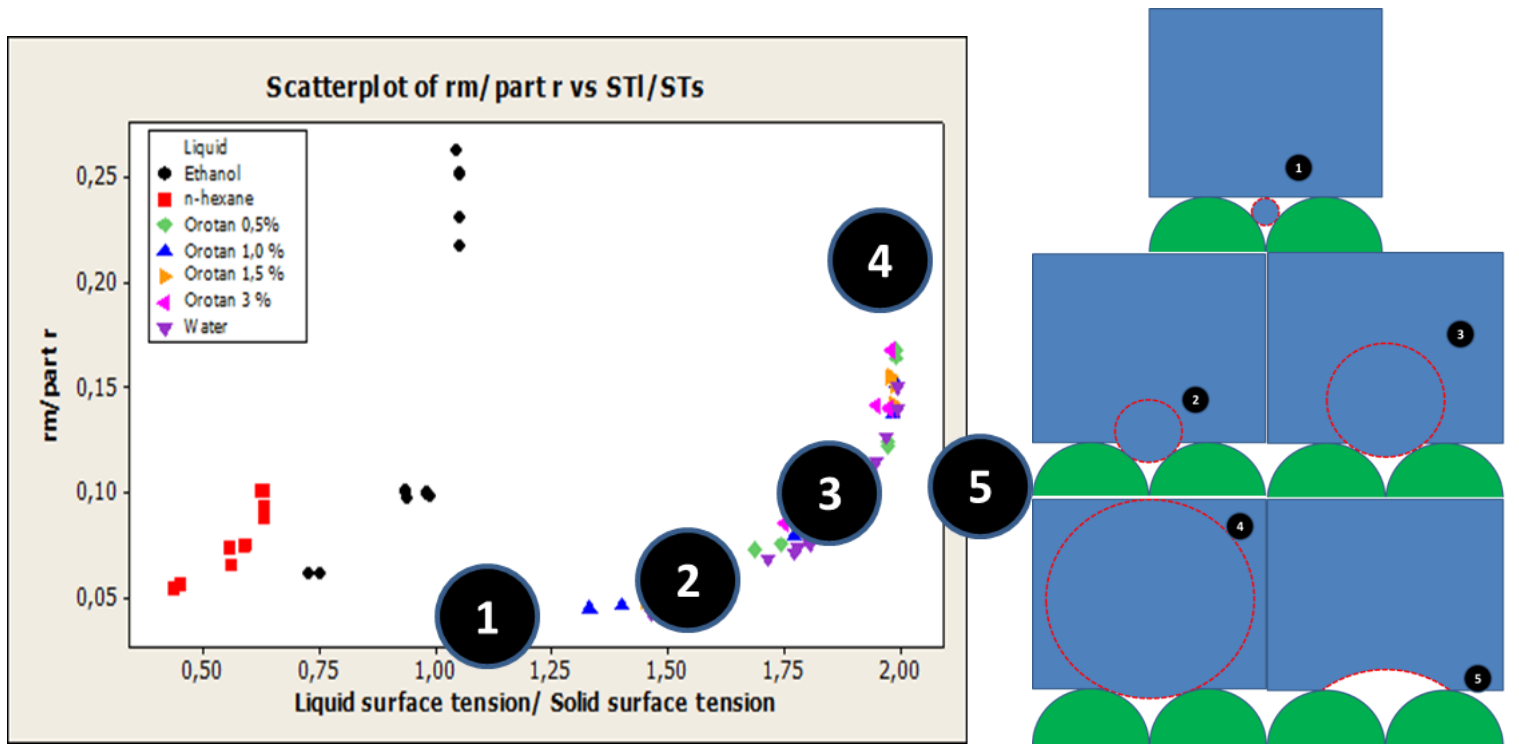

Figure 50 - Didactic demonstration of the correlation between the ratio between meniscus and particle radii and the liquid and solid surface tensions ratio.

The data herein shows that the ratio between solid and liquid surface tensions affects the wettability of the solid by the liquid, and based on the experimental results shown, the data of this study were used to generate a response surface for the wetting speed of the solid by the liquids as a function of the following variables:

Solid to liquid surface tension ratio, $\gamma_{l} / \gamma_{s}$;

Meniscus to particle radii ratio;

Shape factor, $i$.

The objective was to generate an empirical correlation that enables to evaluate the quality of the correlation and the relative importance of these properties. The empirical correlation considered the individual linear and quadratic effect of each variable, and interactions of pairs of the variables. The adjusted $r^{2}$ of the model based on such factors is 0.9546 , which indicates that the model is representative of the wetting process.

Table 19 presents the coefficients of the resulting empirical model generated: 
Table 19 - Coefficients of the cubic empirical model.

\begin{tabular}{|c|c|c|}
\hline & Term & Value \\
\hline \multirow{4}{*}{ } & Constant & -0.0002 \\
\hline & Meniscus/Particle Radii ratio & 0.0314 \\
\hline & Liquid/solid surface tension ratio & -0.0027 \\
\hline & Shape Factor & 0.0007 \\
\hline \multirow{3}{*}{$\begin{array}{l}\frac{0}{\pi} \\
\frac{\pi}{0} \\
\frac{\pi}{20} \\
0\end{array}$} & $\begin{array}{l}\text { Meniscus/Particle Radii ratio* } \\
\text { Liquid/solid surface tension ratio }\end{array}$ & 0.0272 \\
\hline & $\begin{array}{l}\text { Meniscus/Particle Radii ratio* } \\
\text { Shape factor }\end{array}$ & -0.0099 \\
\hline & $\begin{array}{l}\text { Liquid/solid surface tension ratio* } \\
\text { Shape factor }\end{array}$ & 0.0005 \\
\hline$\frac{0}{\frac{0}{2}}$ & $\begin{array}{l}\text { Meniscus/Particle Radii ratio* } \\
\text { Liquid/solid surface tension ratio* } \\
\text { Shape factor }\end{array}$ & -0.0056 \\
\hline
\end{tabular}

The empirical correlation is presented in the Equation 33

WS $=-0.0002-0.0314 \cdot\left(r_{\mathrm{m}} / r\right)-0.0027 \cdot\left(\gamma_{i} / \gamma_{s}\right)+0.0272 \cdot\left[\left(r_{i n} / r\right) \cdot\left(\gamma_{1} / \gamma_{0}\right)\right]-0.0099$ Equatio $\cdot\left[\left(r_{m} / r\right) \cdot i\right\rceil+0.0005 \cdot\left[\left(\gamma_{i} / \gamma_{s}\right) \cdot i\right\rceil-0.0056 \cdot\left[\left(r_{i n} / r\right) \cdot\left(\gamma_{i} / \gamma_{s}\right) \cdot i\right\rceil \quad$ n 33

Response surfaces corresponding to the model are shown in Figure 51. The response surface model was built using three factors. Therefore, to represent changes in the factors and their effect on the wettability process, three curves are made, which represent the three levels in factor $i$ (low, medium and high). Figure 51 shows the three curves from which some conclusions can be drawn.

The first conclusion that can be drawn from the response surfaces in Figure 51 is that, although the shape factor was used to fit the model to the data, it does not affect the response surfaces significantly. The shape of the curve is similar for all levels of the shape factor used in this study. There is a steep slope at the highest levels of the ratio between the meniscus radii and the particle radii. The ratio between liquid and solid surface tension also affects the wetting speed; the lower this ratio, the higher the wetting speed, which means that the lower the liquid surface tension, the higher the wetting speed.

Maximum wetting speed is achieved when the meniscus radius is much smaller than the particle radius. At medium and high levels of the particle shape factor, the wetting speed is affected by the meniscus/particle radii ratio, only. 
Since particles with a shape factor (i) of 4.489 (A and $A^{\prime}$ ) do not have a meniscus particle radii ratio below 0.1 , this part of the plot has no practical meaning.

As seen in Figure 52, the effects that have a statistically relevant influence on the response (wetting speed) are the combination of liquid - solid surface tension ratio and shape factor, the meniscus and particle radii ratio, the shape factor, and the combination of these three factors (meniscus and particle radii ratio, liquid and solid surface tension ratio and the shape factor). Since the liquid and solid surface tension ratio appear in the cubic term, this factor has to be taken into consideration in the model. 


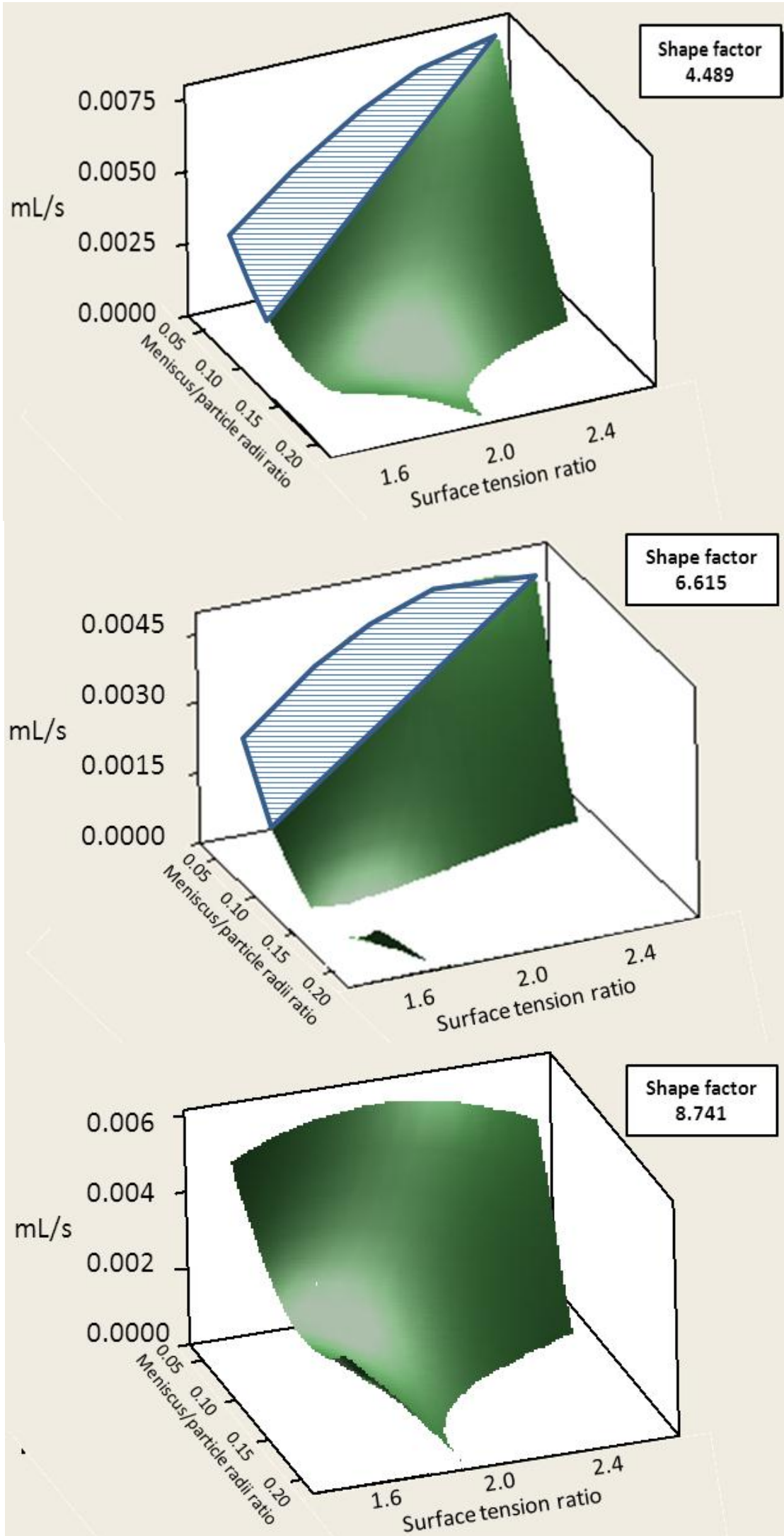

Figure 51 - Response surfaces regression for the wetting speed of particles $A, A^{\prime}, A^{\prime \prime}, B$, and $C$ for shape factors (i) equal to $4.489 ; 6.615$ and 8.741. 


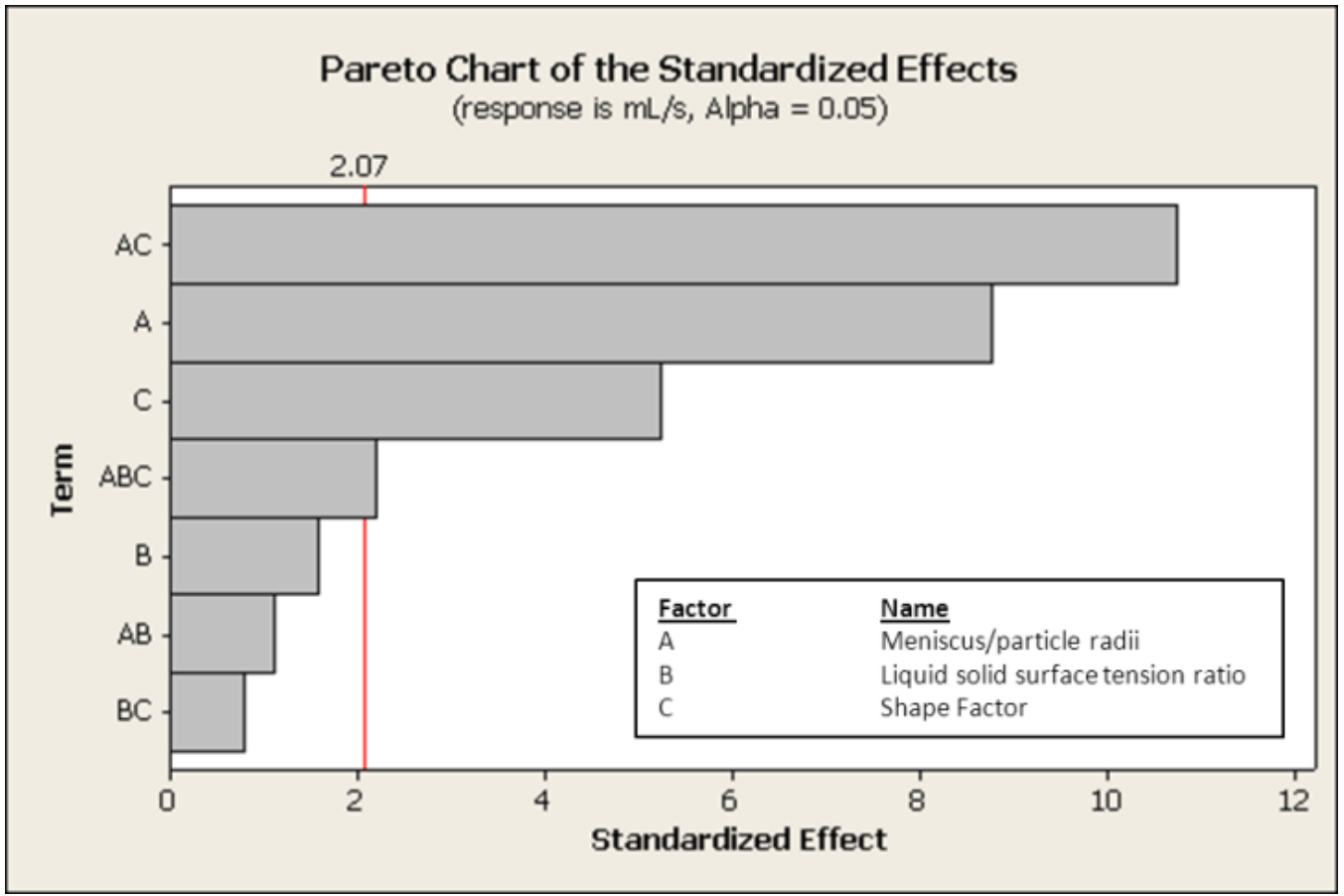

Figure 52 - Pareto chart of the standardized effects in the cubic model.

Although Figure 52 presents the statistically relevant factors and terms, a sensitivity test was carried out in order to verify the response (wetting speed) as a function of the factors. Based on the model coefficients presented in Table 19, the model was reproduced with all variables set to their average value. Each variable was changed individually between minimum and maximum ( 0 and $100 \%$ ) of the range. The change in response (wetting speed) is shown in Figure 54 in terms of the percentage change in its value relative to the range. 


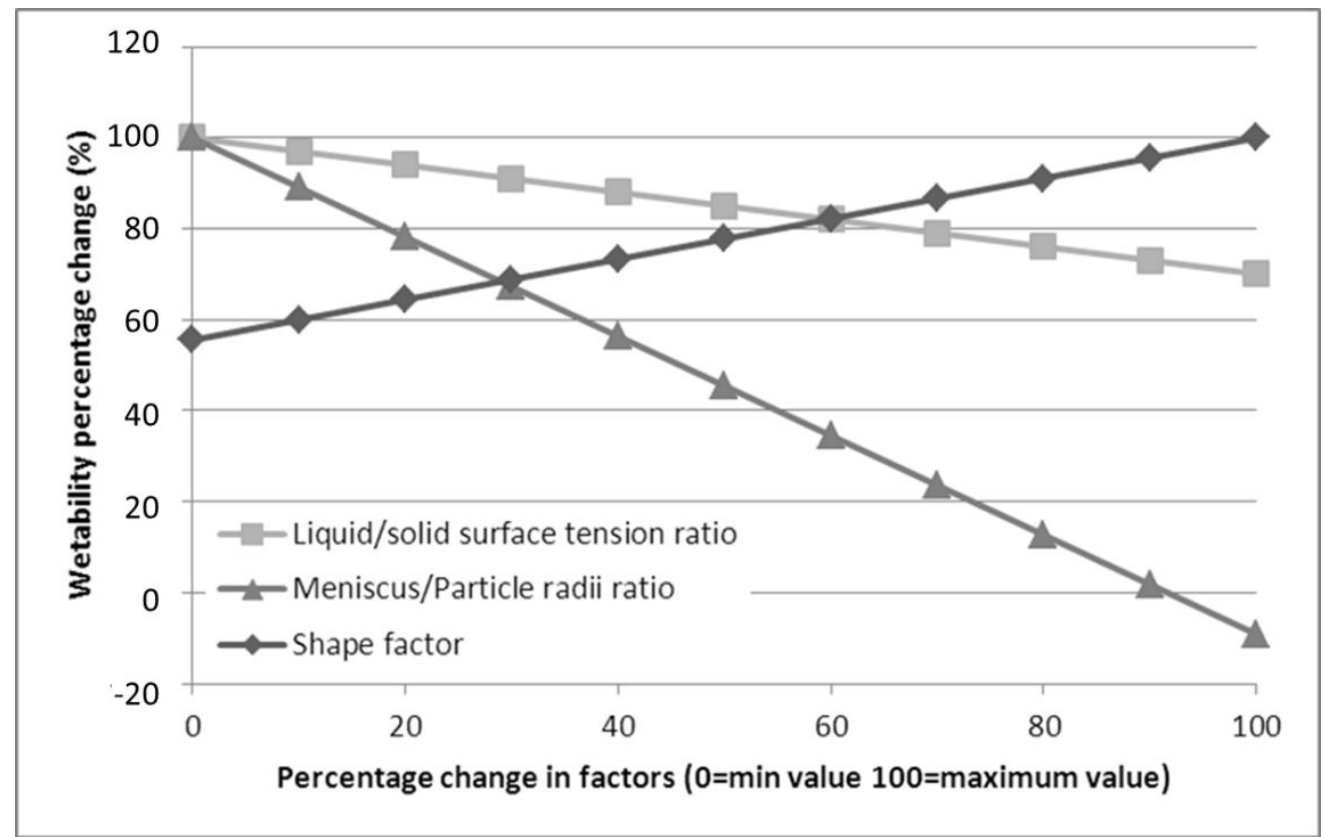

Figure 53 - Sensitivity analysis for the wetting speed for individual changes in the input variables, as predicted by the empirical model.

As shown in Figure 53, the most important factor in wetting speed is the ratio between the meniscus and particle radii. This factor is inversely proportional to the wetting speed and means that if the meniscus is excessively large the wetting speed is greatly reduced. If the particle radius is small enough this ratio is enhanced and, as a consequence, the wetting speed is also reduced. Thus, the meniscus particle radii ratio may represent an important limitation to the wetting speed.

The liquid and solid surface tension ratio is also inversely proportional to the wetting speed, but the effect on the response is smaller when compared to the meniscus and particle radii ratio. There is an interdependency between the meniscus radius and liquid surface tension, as presented in Equation 7. The meniscus is a function of the liquid surface tension and the capillary pressure.

The particle radius also influences the radii ratio factor. The smaller the particle, the larger is the factor and smaller is the wetting speed. Therefore, if the rule is extrapolated to nanometric size particles the wetting speed of the agglomerates is further reduced or even stopped.

The shape factor s proportional to the wetting speed. The larger the shape factor, the larger is the wetting speed, meaning that for larger shape factor, the capillary radius is smaller, and the capillary pressure is higher, as shown in Figure 54. 


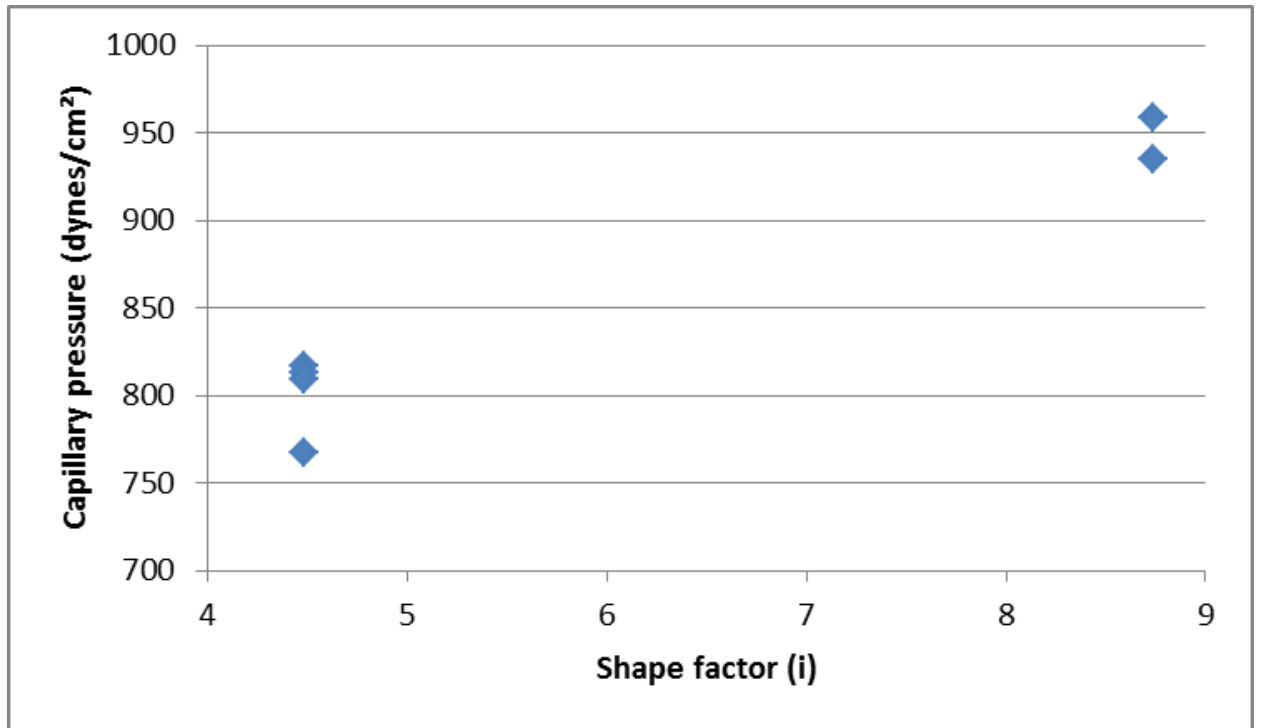

Figure 54 - Capillary pressure as a function of shape factor (i).

The elevated capillary pressure causes a faster penetration of the liquid into the agglomerates, but this factor is less important than the ratio between the meniscus and particle radii.

With the information above, it is possible to calculate the work of dispersion (sum of the work of adhesion, $W_{a}$, the work of penetration, $W_{p}$, and the work of spreading, $\mathrm{W}_{\mathrm{s}}$ ) (see Table 20). A positive work of dispersion denotes that the dispersion is spontaneous, and the higher the work of dispersion, the more spontaneous is the dispersion.

Table 20 - Continues Work of dispersion from the powders and liquids used in this study

\begin{tabular}{|c|c|c|c|c|c|c|c|c|c|c|c|c|}
\hline & \multicolumn{4}{|c|}{ n-hexane } & \multicolumn{4}{|c|}{ Water } & \multicolumn{4}{|c|}{ Dispersant $0,5 \%$} \\
\hline & Wa & Wp & Ws & $\begin{array}{c}\text { Work of } \\
\text { dispersion }\end{array}$ & Wa & Wp & Ws & $\begin{array}{c}\text { Work of } \\
\text { dispersion }\end{array}$ & Wa & Wp & Ws & $\begin{array}{l}\text { Work of } \\
\text { dispersion }\end{array}$ \\
\hline A & 100.2 & 82.2 & 62.8 & 245.1 & 80.2 & 7.2 & -65.9 & 21.5 & 60.2 & 1.4 & -57.5 & 4.1 \\
\hline$A^{\prime}$ & 97.4 & 79.4 & 62.8 & 239.6 & 74.0 & 1.0 & -72.0 & 3.1 & 62.5 & 3.6 & -55.2 & 10.8 \\
\hline A" & 175.3 & 157.3 & 139.3 & 472.0 & 268.0 & 195.0 & 122.0 & 584.9 & 443.0 & 384.2 & 325.3 & 1152.6 \\
\hline B & 153.7 & 135.7 & 115.7 & 405.1 & 137.2 & 64.2 & -9.8 & 191.6 & 89.3 & 30.4 & -28.9 & 90.8 \\
\hline C & 149.8 & 131.8 & 115.7 & 397.3 & 152.0 & 79.0 & 7.2 & 238.2 & 130.9 & 72.0 & 14.2 & 217.1 \\
\hline
\end{tabular}


Table 20 - Conclusion work of dispersion from the powders and liquids used in this study

\begin{tabular}{|c|c|c|c|c|c|c|c|c|c|c|c|c|}
\hline & \multicolumn{4}{|c|}{ Dispersant 1,0 \% } & \multicolumn{4}{|c|}{ Dispersant 1,5\% } & \multicolumn{4}{|c|}{ Dispersant $3 \%$} \\
\hline & Wa & Wp & Ws & $\begin{array}{l}\text { Work of } \\
\text { dispersion }\end{array}$ & Wa & Wp & Ws & $\begin{array}{l}\text { Work of } \\
\text { dispersion }\end{array}$ & Wa & Wp & Ws & $\begin{array}{l}\text { Work of } \\
\text { dispersion }\end{array}$ \\
\hline A & 54.9 & 0.5 & -54.0 & 1.4 & 53.5 & 2.6 & -48.4 & 7.7 & 49.3 & 3.7 & -41.9 & 11.2 \\
\hline$A^{\prime}$ & 56.0 & 1.6 & -52.8 & 4.8 & 52.5 & 1.6 & -49.3 & 4.8 & 48.0 & 2.5 & -43.0 & 7.4 \\
\hline$A^{\prime \prime}$ & 275.6 & 221.1 & 166.7 & 663.4 & 220.6 & 169.7 & 118.8 & 509.1 & 150.7 & 105.2 & 59.6 & 315.5 \\
\hline B & 81.5 & 27.0 & -27.8 & 80.7 & 79.6 & 28.7 & -22.7 & 85.5 & 60.2 & 14.6 & -31.1 & 43.7 \\
\hline C & 103.2 & 48.8 & -5.0 & 147.1 & 86.6 & 35.6 & -14.8 & 107.4 & 91.0 & 45.5 & 0.6 & 137.2 \\
\hline
\end{tabular}

Figure 55 presents the correlation between the work of dispersion calculated for each one of the tested liquids and the percentage of the volume occupied by these liquids relative to the pendular volume or total pore volue (volume occupied by the $n$ hexane). The dispersion becomes more spontaneous as the liquid occupies a higher percentage of the agglomerate pores volume.

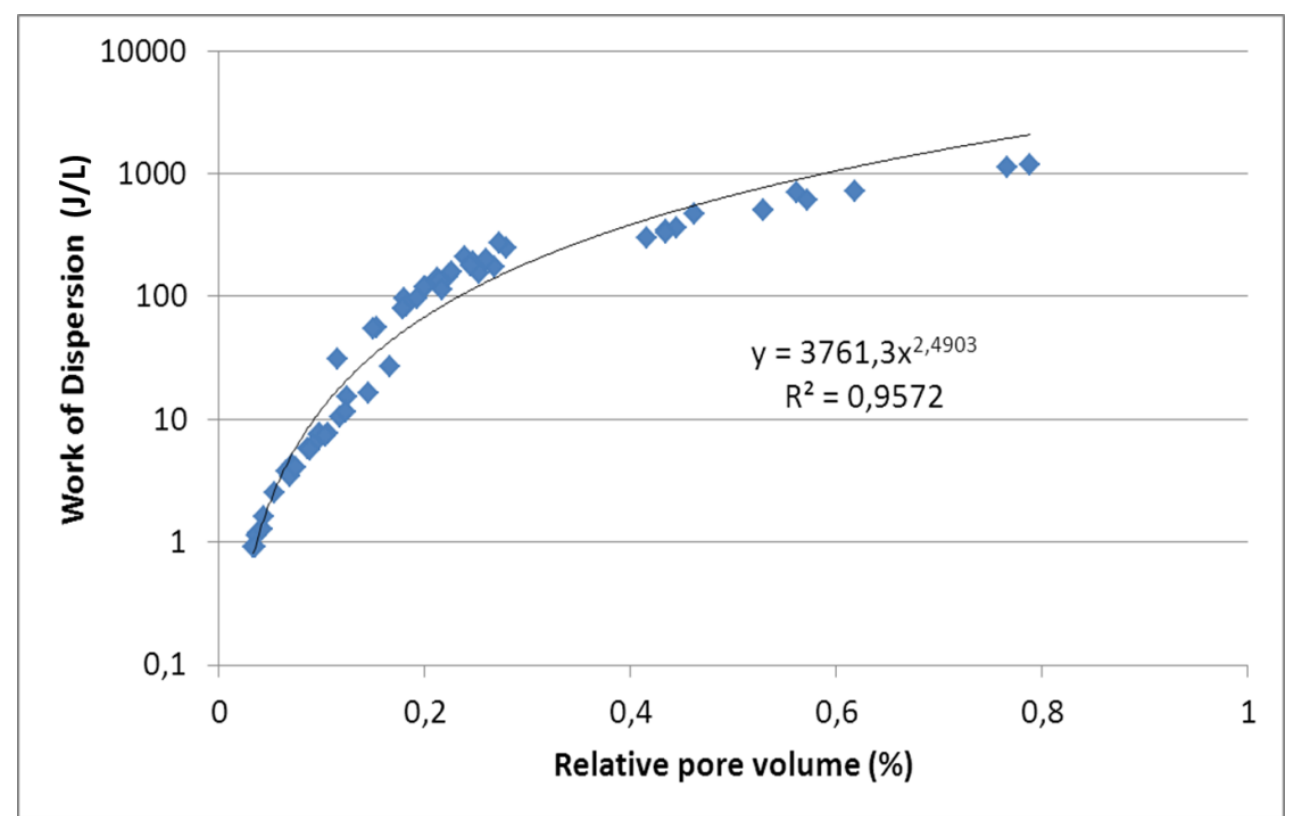

Figure 55 - Work of dispersion as a function of the relative volume of the pore occupied by the liquid penetration into the agglomerate

\subsection{Deagglomeration energy}

The next part of this study is dedicated to the study of the incorporation of the $\mathrm{TiO}_{2}$ particles into the liquid medium. The objective of this part of the study is to understand how the particle size distribution changes during the dispersion process, when the solids are subject to a controlled shear stress applied by the ultrasound generator. The applied ultrasound energy was quantified, and a correlation between the energy applied and the other particle and liquid parameters is proposed. Thus, 
this part of the study is focused on correlating the particle wetting properties with the energy involved in the dispersion process.

The experiments consisted of monitoring the particle size distribution (by laser diffraction) over time with the ultrasound generator placed inside the dispersion recipient and operating at a constant power, until no change in the particle size distribution curves could be observed. This was also controlled by monitoring the parameters of the particle size distribution, like $D(4,3)$ and $D(3,2)$.

The changes in the particle size distribution curves of the samples used in this study are shown in Figure 56 when water was used, for different values of the accumulated ultrasound energy applied. For all samples, during the dispersion process the larger agglomerates disappear gradually, and most of the particles tend to stabilize in the form of smaller agglomerates. For sample A", which initially showed a spread particle size distribution curve, the disappearance of peaks at specific sizes is also observed during the dispersion.

The deagglomeration energy was obtained for each sample when it was submitted to dispersion in different liquids. Figure 57 shows a compilation of the energy required to deagglomerate the samples until a stable particle size distribution was attained in liquids with different values of the surface tension, for particles with different shape factors. The results show that the liquid surface tension plays an important role in determining the required energy to achieve minimum particle size. Apparently there is a critical value for the liquid surface tension, above which the dispersion process requires a significantly larger energy and the particle shape factor affects the process. Figure 57 shows that the drop in required energy when changing the liquid from pure water to water plus dispersant additive is significant. 


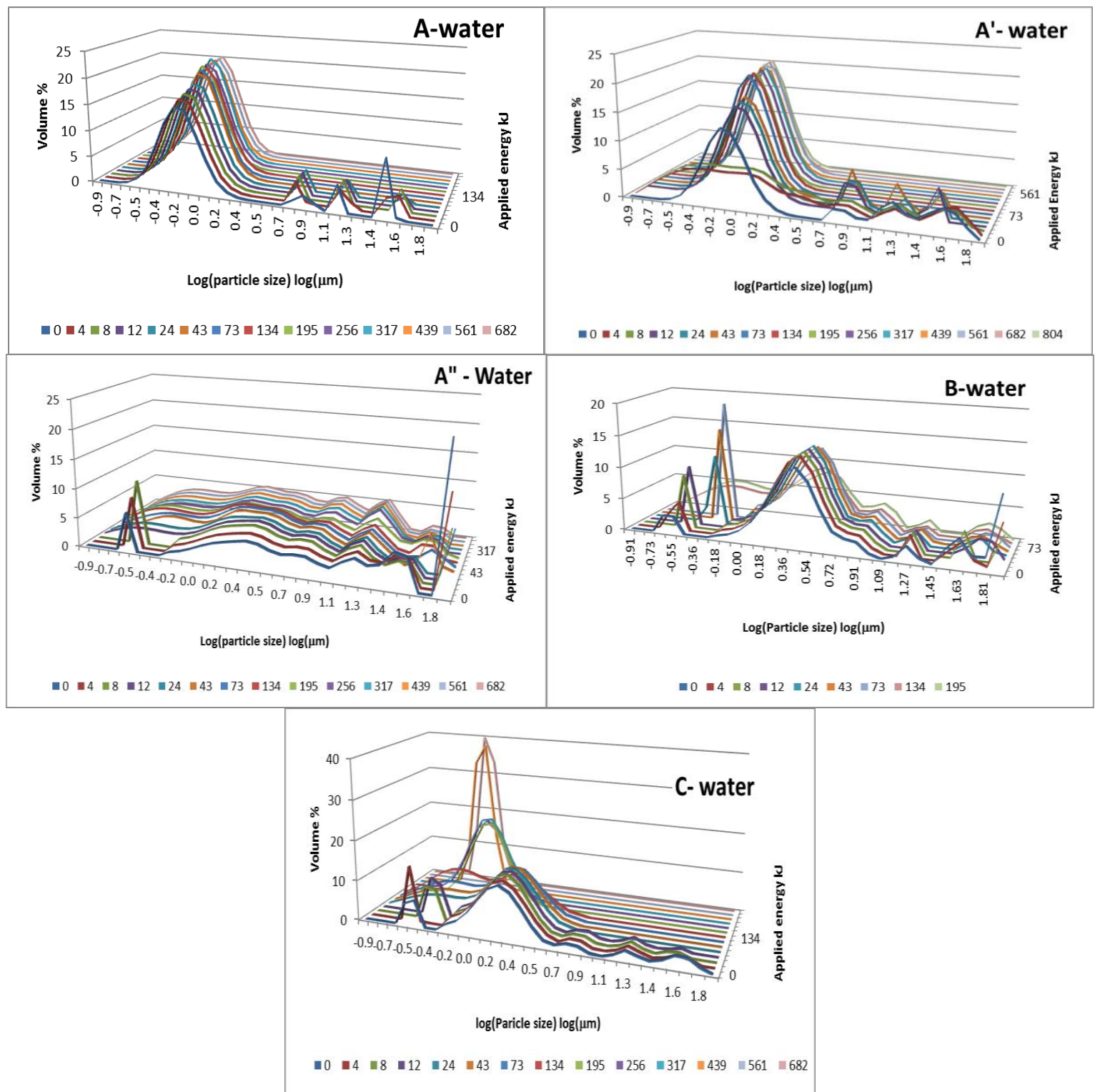

Figure 56 - Particle size distribution for different values of ultrasound energy applied to the samples used in this study (particles dispersed in water). 


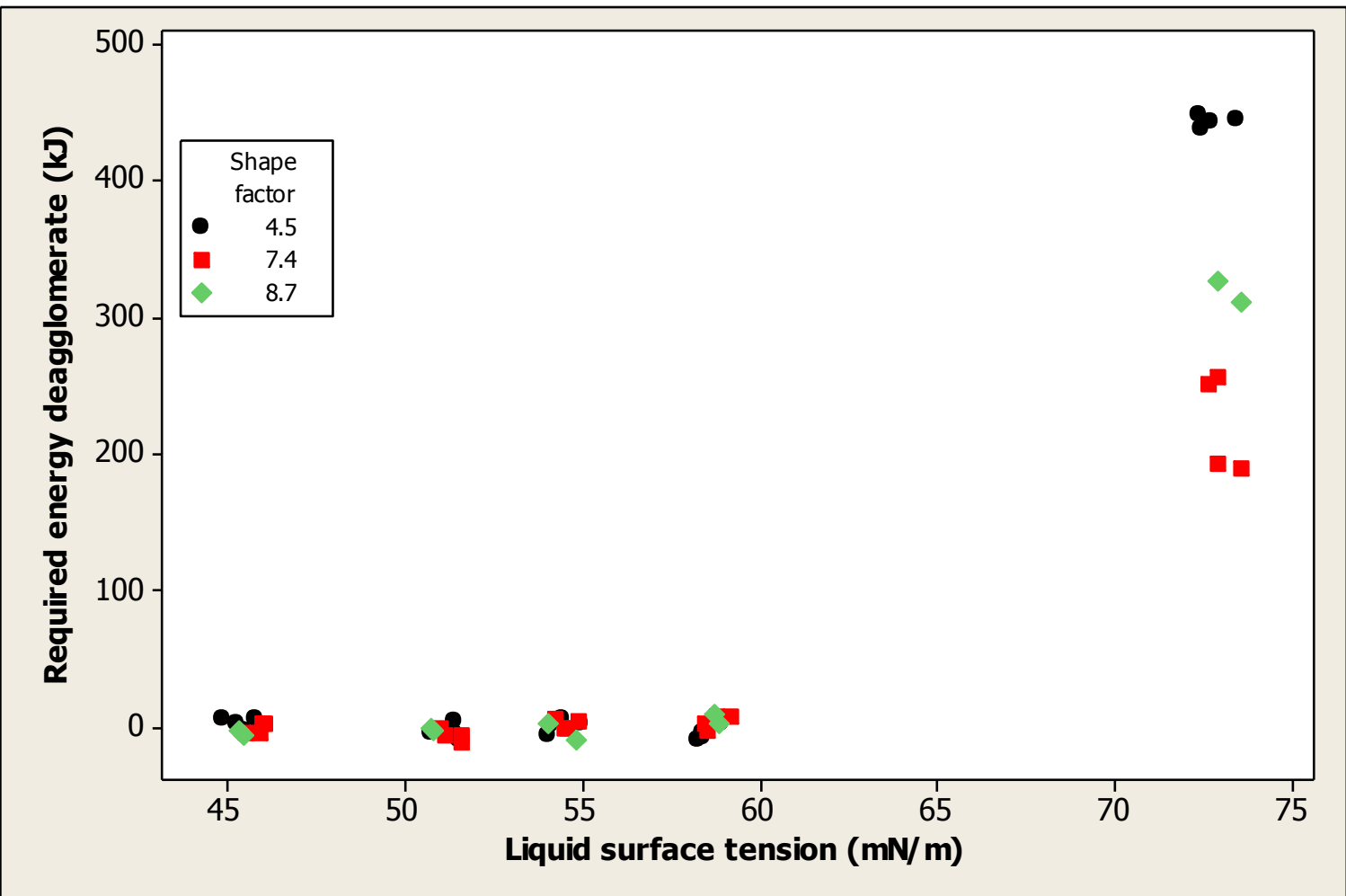

Figure 57 - Scatter plot of energy required in the dispersion for liquids with different surface tension and particles with different shape factor (i).

The graph shown in Figure 58 was built with the data collected from the dispersion experiments under the effect of ultrasound. The $x$ axis represents the liquid surface tension, and the $y$ axis is the energy applied to reduce the mean particle size, $D(4,3)$, to its minimum observed value.

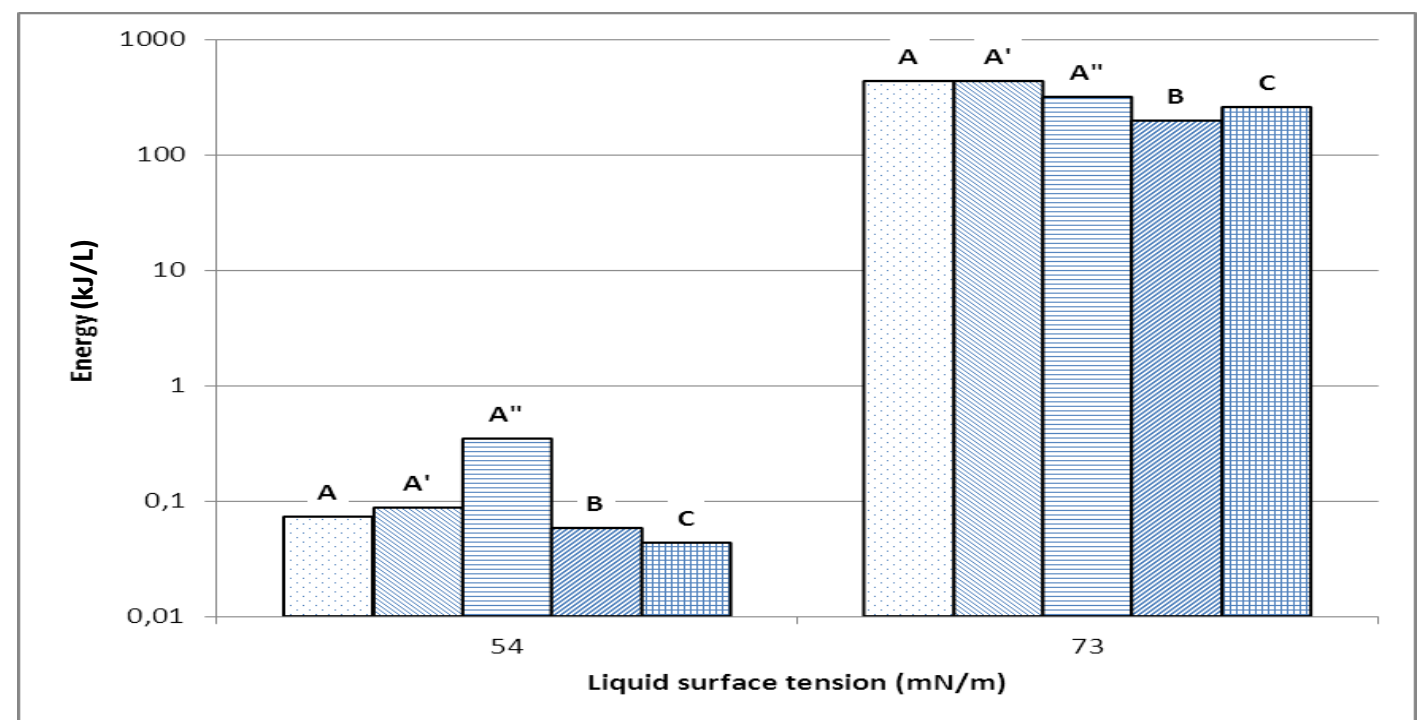

Figure 58 - Ultrasound energy required to achieve minimum mean particle size, $D(4,3)$, for the samples used in this study. 
Figure 58 shows that particles presenting different surface properties behave differently. Since particles A, $A^{\prime}$, and A" have the same surface treatment, they were expected to behave similarly. However, this is true for particles $A$ and $A^{\prime}$, only. The difference observed for sample A" may be due to its different particle size distribution. When the distribution of particle size is spread over a wide size range, the particle-to-particle interaction is much larger than for monodisperse particles, since the void space is smaller. The particle size distribution curves of all samples used in this study is shown again in Figure 59. Sample A" shows a significantly different particle size distribution curve compared to the other samples.

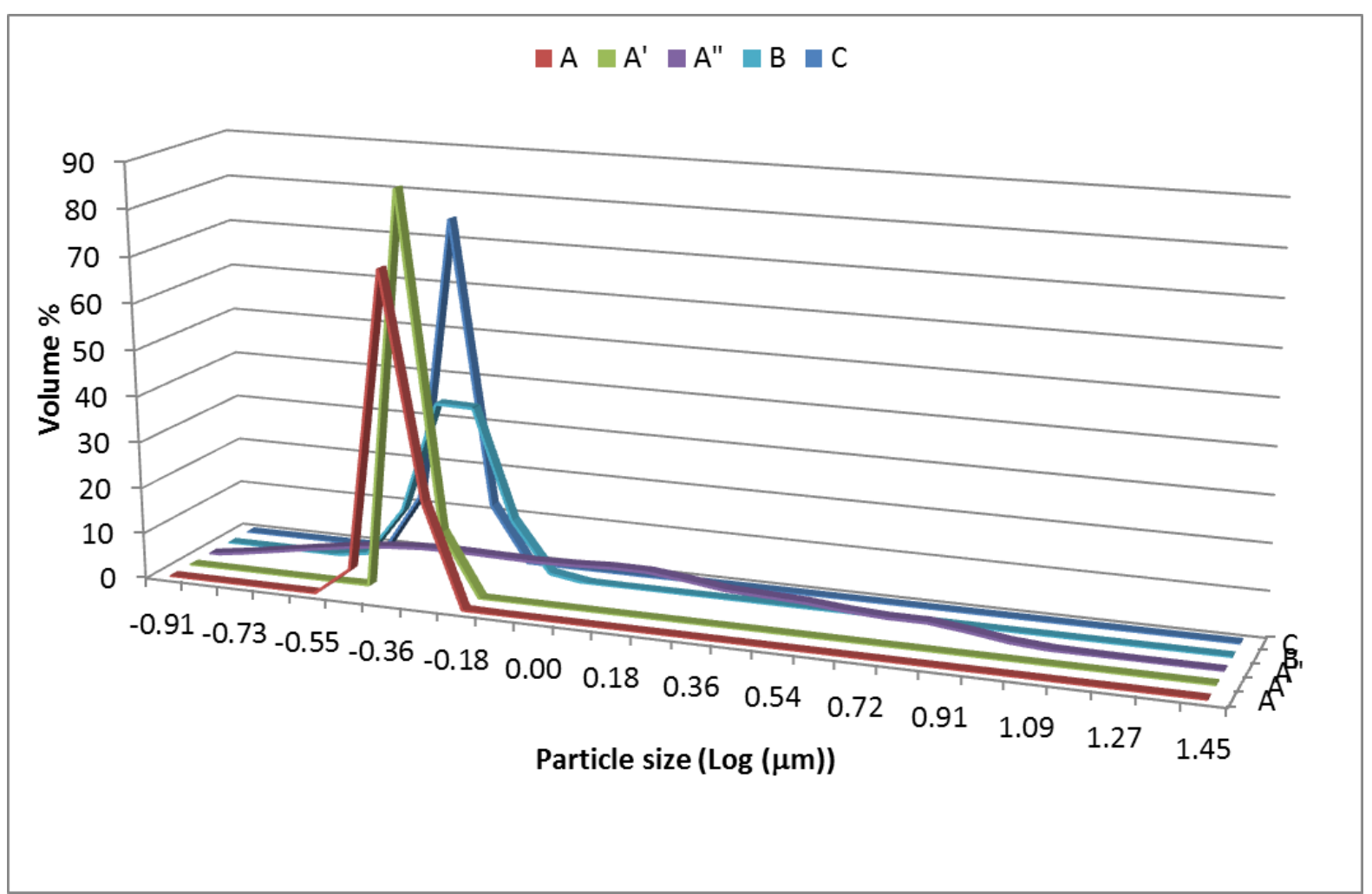

Figure 59 - Particle size distribution of samples A, A', A", B, and C.

The relationship between energy required for dispersion and the properties of the liquids and particles can be visualized in Figure 60, which shows the same information shown in Figure 57, but in the form of a log-log plot. The important information shown in Figure 60 is that the required energy for dispersion can be correlated to the liquid surface tension logarithmically. 


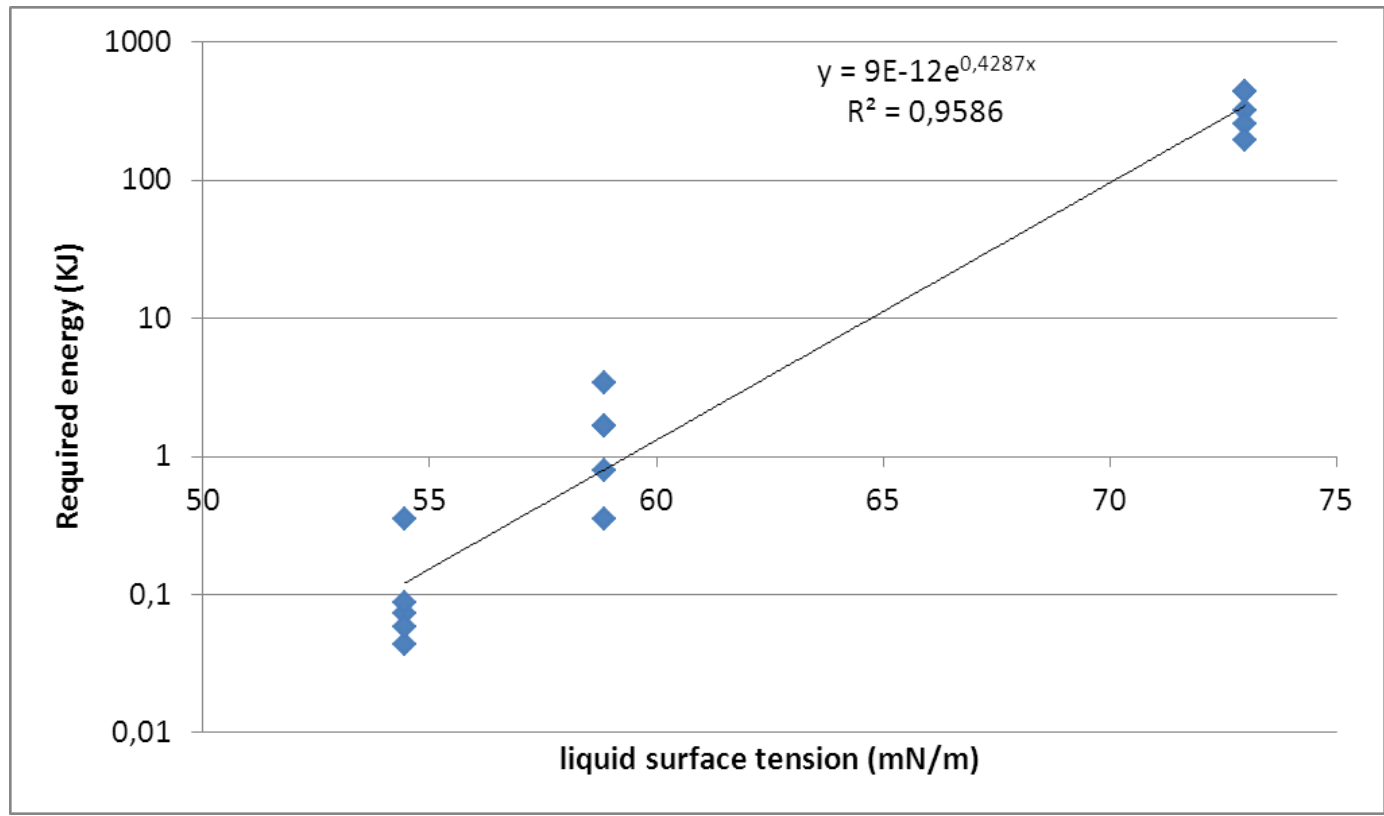

Figure 60 - Required energy as a function of the liquid surface tension for all samples.

In Figure 60 the data are plotted in a similar log-log scale, but specific correlations are shown for each value of the particle shape factor (i). In this case, the fitting became significantly better. The plots show that the slope of the fitted straight lines varies with the particle shape factor. Based on this, the parameters of the equations shown in Figure 60, i.e. slope and exponent, are plotted as a function of the particle shape factor, I, in Figures 61 and 62.

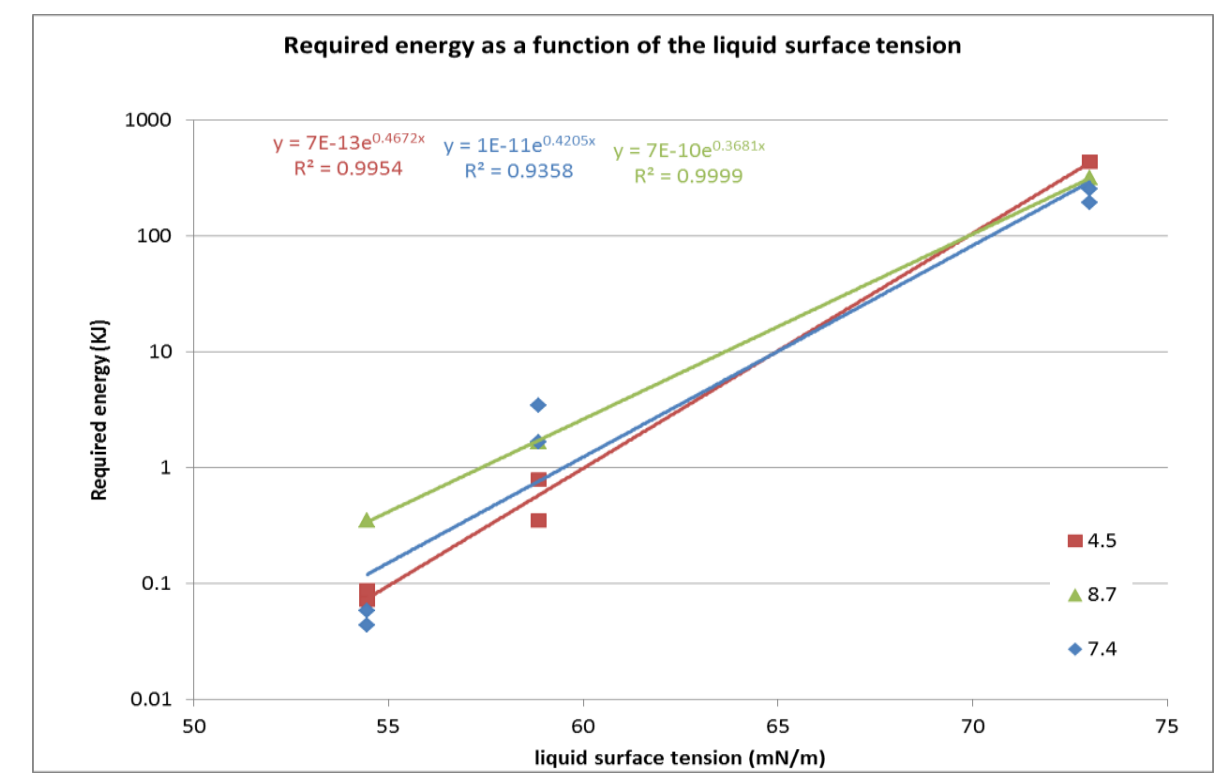

Figure 61 - Required surface tension as a function of liquid surface tension and the particle shape factor i $(4.5 ; 8.7$ and 7.4$)$. 
The observed relationship between the exponent of the equations in Figure 61 and particle shape factor denotes that the rougher the particle, the more energy is required to disperse the particles in lower liquid surface tensions.

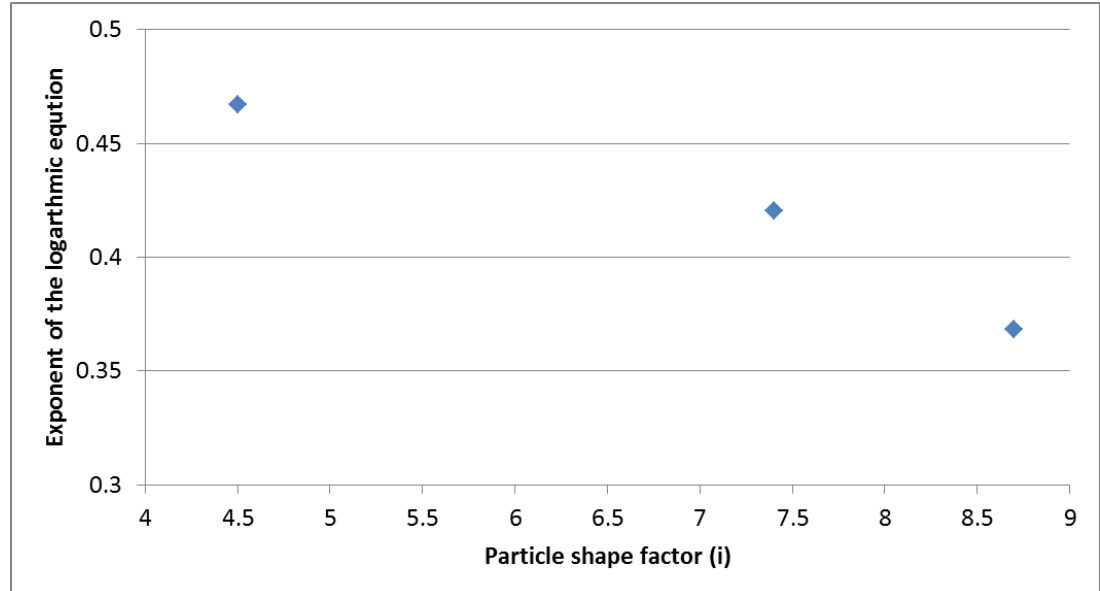

Figure 62 - Exponent of the logarithmic equations in Figure 60 as a function of the particle shape factor, i.

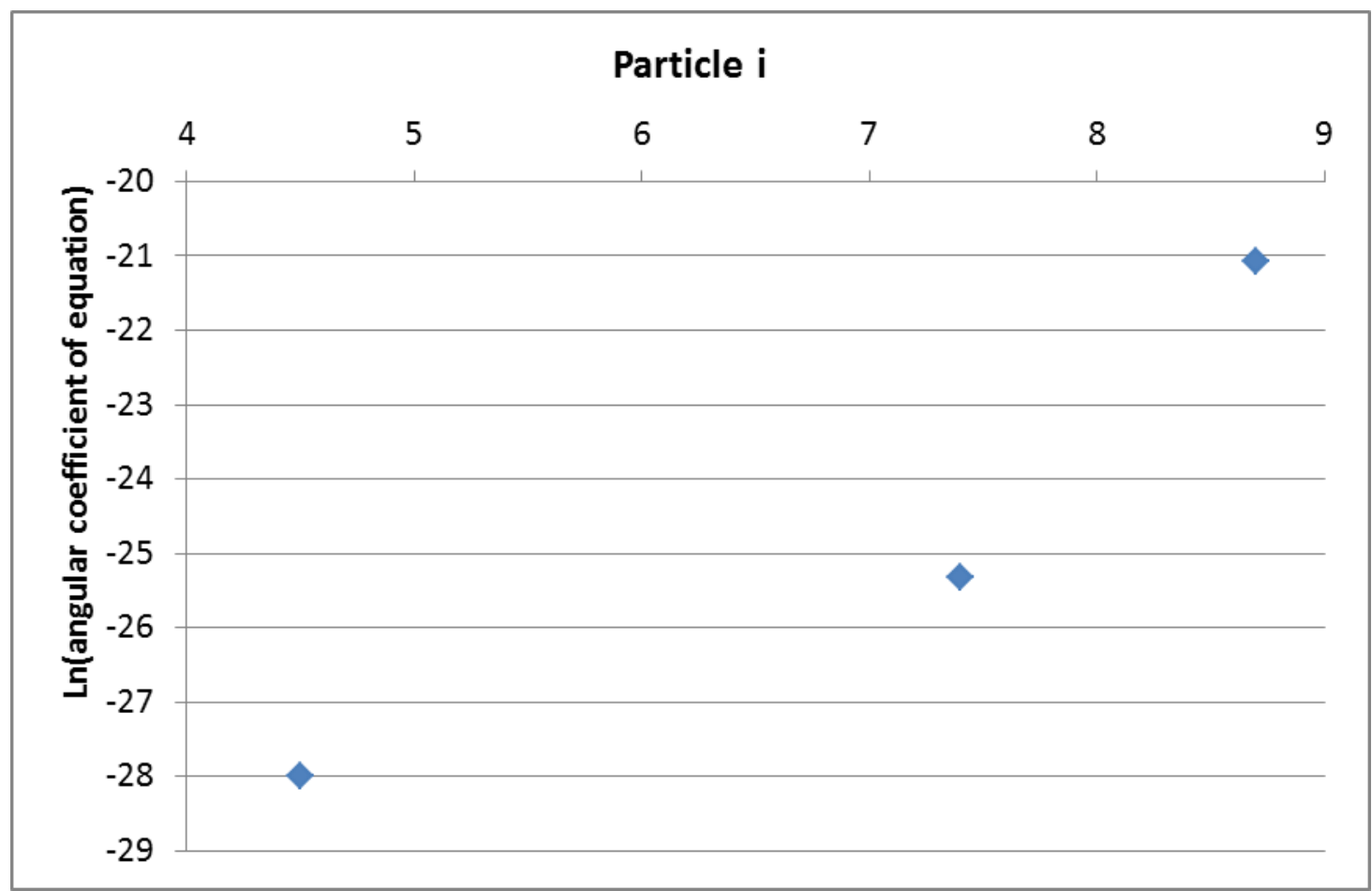

Figure 63 - Angular coefficient as a function of the particle shape factor

Based on the mentioned correlations, an empirical expression was adjusted to the data. For the particles in this study, the required energy (RE) as a function of the liquid surface tension and particle shape factor were correlated according to Equation 34:

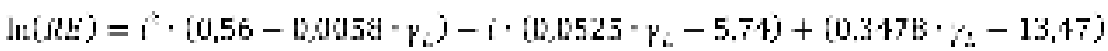


Equation 34 can be applied to predict the energy required to achieve the minimum mean particle size for the particle samples used in this study. A comparison between predicted and observed values of the required energy is shown in Figure 64. As shown in the figure, the agreement between observed and predicted values is good.

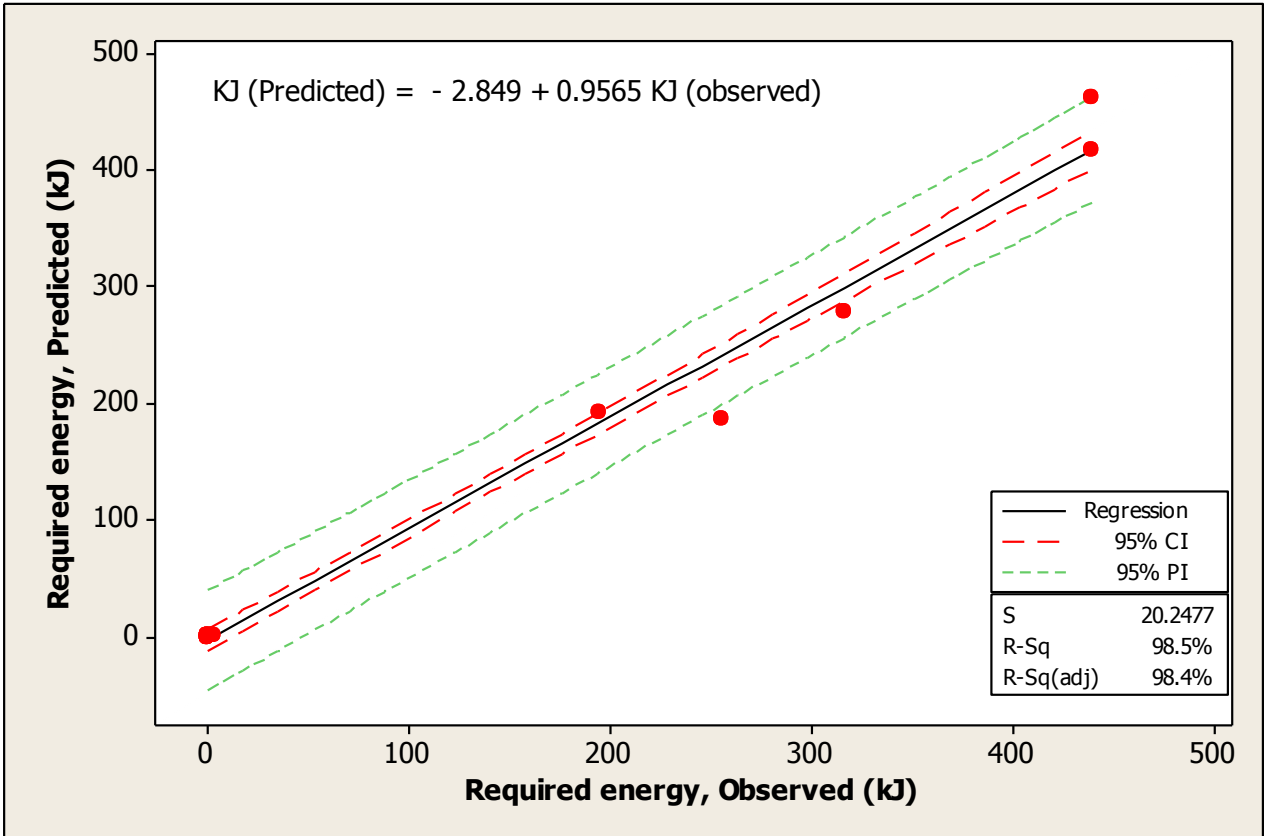

Figure 64 - Comparison between observed and predicted values of required energy for dispersion of the $\mathrm{TiO}_{2}$ particles.

The same results in Figure 64 are presented in figure 66 with the axes in logarithmic scale.

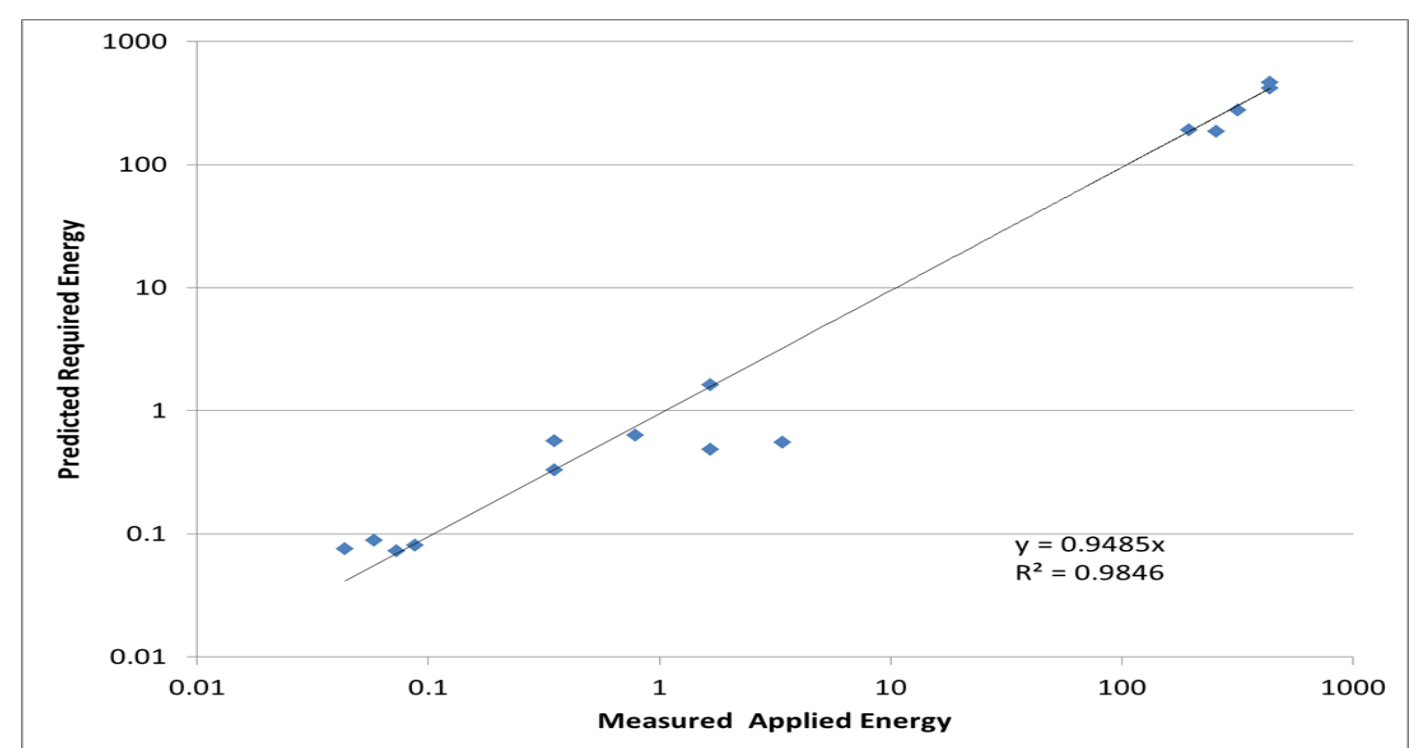

Figure 65 - Comparison between predicted values of required energy for dispersion of the $\mathrm{TiO}_{2}$ particles (log scales)

Figure 66 shows the correlation between the required deagglomeration energy and the relative agglomerate maximum tensile strength calculated by means of Equation 
14. The maximum tensile strength apparently corresponds to the potential required energy to deagglomerate the samples for the three values of the shape factor, 4.5 , 7.4 and 8.7, used in this study. It is possible to observe from Figure 66 that the particle shape plays an important role in the energy required to reduce the mean particle size to its minimum value.

By taking as reference the shape factor value of 4.5 , the sample with shape factor equal to 8.7 requires more energy to deagglomerate than the reference, and the sample with shape factor equal to 7.4 requires less energy than the reference to achieve the minimum particle size. This behavior can be explained by taking into account the origin of the particles. In this study, particles with shape factor 8.7 are the same particles with shape factor 4.5 but with no micronization, meaning that the particles are attached to each other physically. This physical attachment changes the shape of the particles but not the surface roughness, meaning that the average number of particle-to-particle interaction per particle increases. In this particular case, most particles of the sample consist of aggregates and not of single unitary primary particles (that have no, or too little physical bounding to each other).

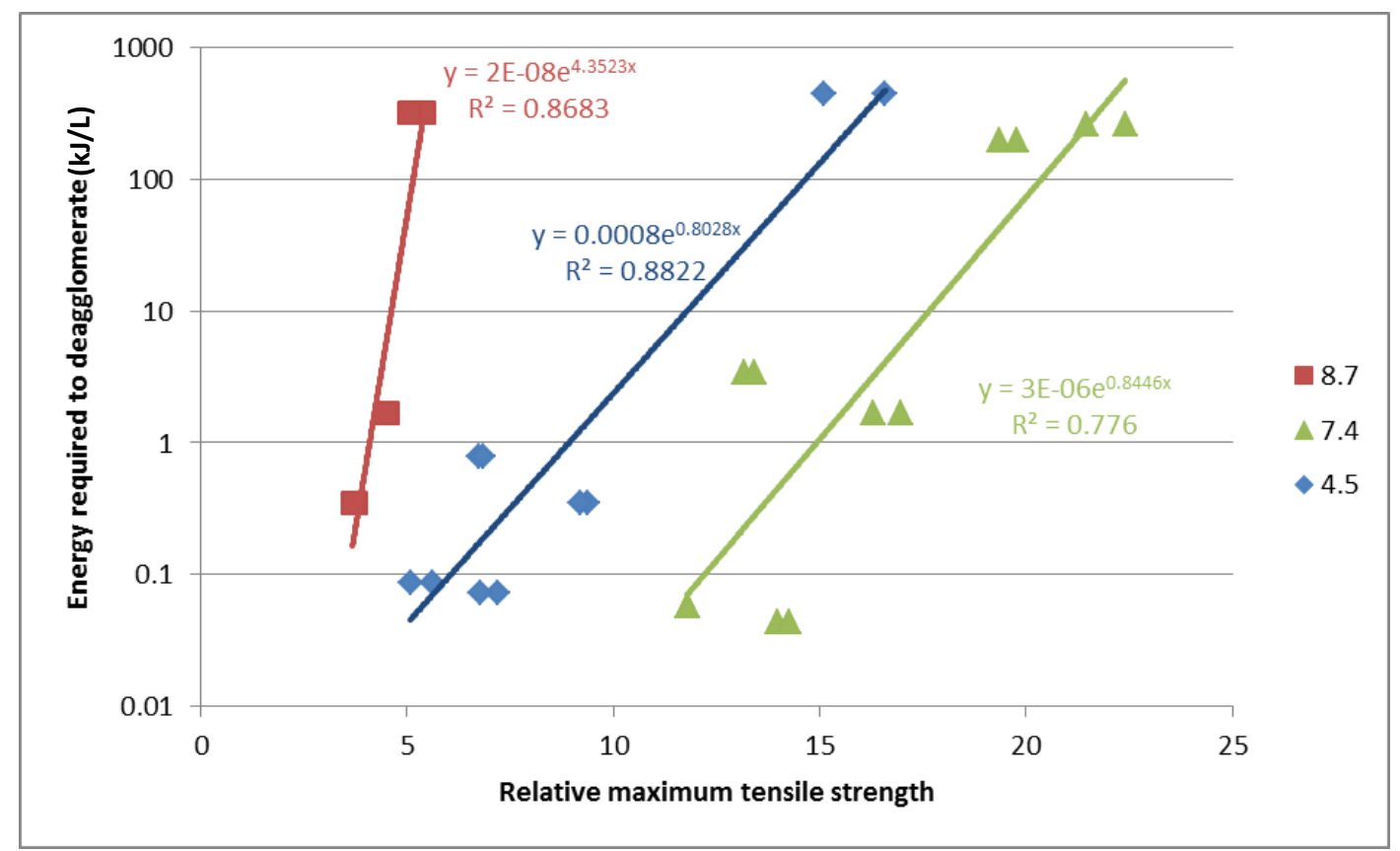

Figure 66 - Correlation between calculated relative agglomerates tensile strength and required energy to deagglomerate the powder.

This configuration is illustrated in Figure 67, which presents a scanning electronic microscope image of a typical pigment grade $\mathrm{TiO}_{2}$ sample. The image shows unitary 
particles and aggregates. The unitary particles are circled by a solid line (1) and the aggregates are circled by a dashed line (2). The roughness of the particles is not visible from this magnification, but the shape of the aggregates promotes interparticle interaction. Thus, the aggregates in the samples may be responsible for the additional resistance to the deagglomeration process, and this is caused by particle shape, since, as shown in the image, such irregular particles present a large contact area in the aggregates.

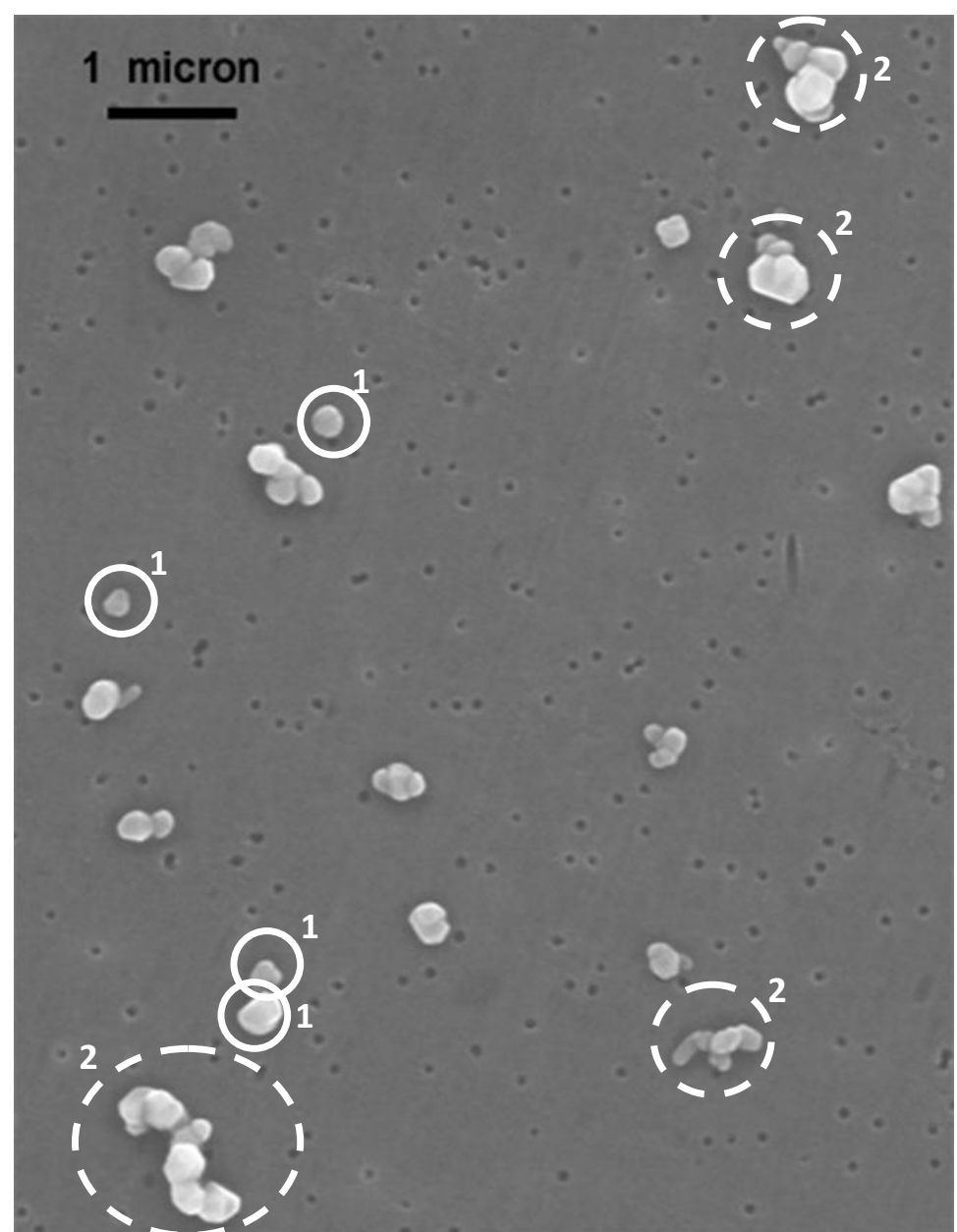

Figure 67 - Scanning electron microscope image of a typical micronized $\mathrm{TiO}_{2}$ sample. 1- unitary particles 2- aggregates. .(Dupont, internal technical document, authorized for this study)

The results of the experiments on wetting and dispersion can be summarized in a qualitative way in the form of the diagram in Figure 68. The left column illustrates the behavior of individual particles: as the particles become less spherical, tending to a dendritic structure, the sphericity decreases and the shape factor, i, increases. This tendency is associated with a reduction of interparticle contact, resulting in lower values of the required energy in dispersion operations. 
The behavior is different for agglomerates of irregular particles. As the particles become less spherical, sphericity decreases, and the shape factor increases. However, as illustrated in Figure 67, agglomerates of irregular particles tend to exhibit larger interparticle contact areas, and this, results in an increased resistance to deagglomerate. Thus, the required energy for achieving a minimum mean particle size in dispersion operations is increased.

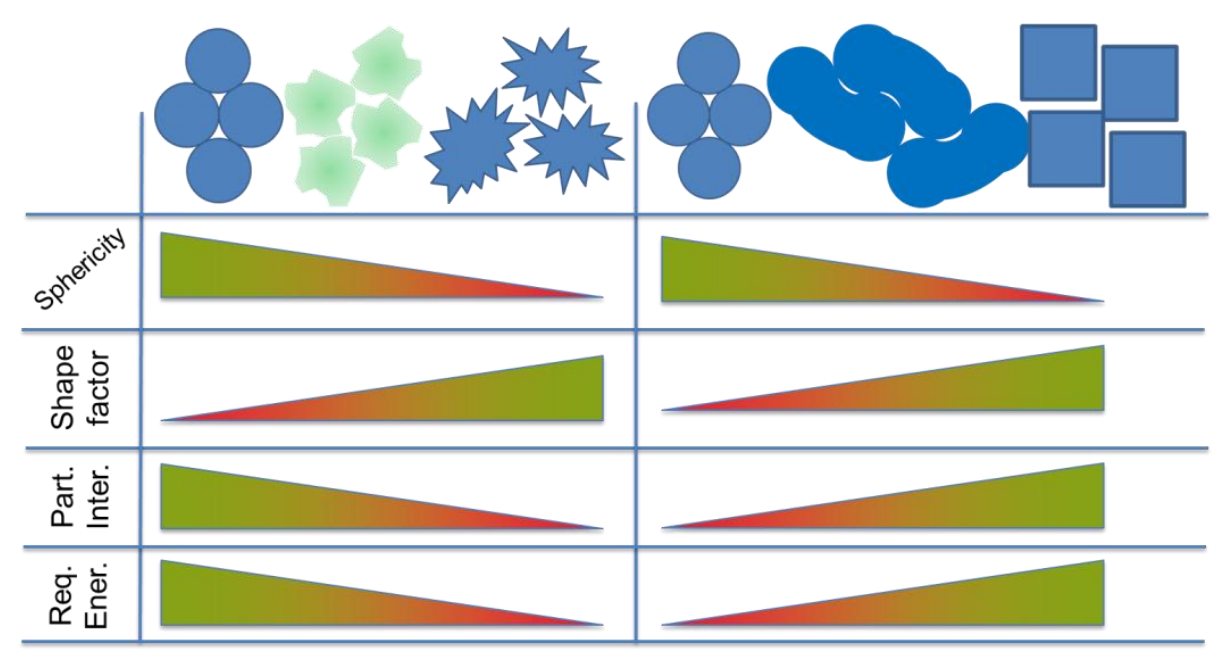

Figure 68 - Qualitative sketch to summarize the effect the particle shape on the required energy to achieve minimum mean particle size. 


\section{Conclusions}

A set of characterization experiments was carried out with five samples of pigment grade titanium oxide particles which are representative of commercially available products. The experiments were aimed at obtaining correlations between pertinent particle properties and their behavior in relation to the preparation of liquid suspensions, which involves wetting and deagglomeration processes.

The wetting speed of the agglomerates was studied as well as the energy required to reduce the size of the particles to their minimum value, thus achieving maximum dispersion. The factors influencing these two phenomena were evaluated, and the most important ones were identified.

Three main factors influence the wetting speed. The first one is the relationship between the particle size and the meniscus formed by the liquid when it penetrates the pores of the agglomerates. The second is the relationship between the liquid and the solid surface tensions. A third factor that also influences the wetting speed is the shape factor of the particles, which was also included in the set of variables used to adjust an empirical correlation for predicting the wetting speed of different particles.

The influence of particle size and the meniscus radius can be defined as follows: If the meniscus is small enough to allow permeability throughout the pores formed by the particles, the wetting process occurs. If the particle accommodation or packing forms pores smaller than the meniscus that is formed by the liquid, then the wetting process is slowed or even stopped.

The influence of the ratio between liquid and solid surface tensions is predicted in the literature reviewed in Chapter 2. The higher the surface tension of the liquid compared to the surface tension of the solid, the lower the wetting speed. This relation also applies to the formation of the meniscus radius and the phenomena of surface interaction. The ratio of the surface tensions is a most important factor in the wetting process.

The shape of the particles is also important in determining the required energy in dispersion because it defines the number of interactions among the particles. The larger the number of interactions, the harder it is to deagglomerate the particles. In 
this study, the shape factor of particles of sample A (A, A' and A") was larger than the others, which resulted in an the increase in required energy.

The properties of the liquid influence the deagglomeration process, mainly. Patton (1979) predicted the effect of liquid surface tension and viscosity, particle-related properties, although always associated with a property of the liquid, play an important role in the wetting behavior (particle radius, particle shape factor (i), agglomerate pore volume, and the specific surface area).

The maximum mass adsorbed by an agglomerate is proportional to the liquid surface tension. The adsorption speed of the liquid volume is clearly a function of the ratio between the liquid and solid surface tensions and the shape factor. In this case, the lower the liquid surface energy in relation to the solid surface tension for any roughness value (i), the greater the speed of the adsorbed volume.

Addition of dispersant causes an increase in the observed viscosity, which imposes resistance to the liquid penetrating the agglomerates pores, tending to reduce the wetting speed. If the capillary pressure is increased, the penetration speed also increases. The capillary pressure is a function of the liquid surface tension. The higher the surface tension is, the higher the capillary pressure. Thus, an enhanced penetration speed is expected if the liquid surface tension is decreased.

The wetting speed is influenced by the capillary volume, which is determined by the diameter of the void volumes in the agglomerates. The liquid surface tension is predicted by the theory presented by Patton (1979), but it is also highly influenced by the interaction between the liquid and solid surface tensions. The greater their affinity is, the easier the wetting and the lower the energy required.

A correlation between particle and liquid properties and the required energy to disperse the particles was adjusted to the experimental results. The correlation (Equation 31) can predict the energy required to achieve maximum deagglomeration of pigmentary $\mathrm{TiO}_{2}$ powders for the samples used in this study. The energy required for pigment dispersion is described as a function of the ratio between the liquid and the particle surface tensions and the particle shape factor. The energy required decreases as this ratio decreases. This ratio is driven by changes in the liquid surface tension because the solid surface tension does not change during the 
process. Therefore, this ratio is an important factor for the dispersion process of pigmentary $\mathrm{TiO}_{2}$ particles. 


\section{References}

Abrahão, R. T., 2007. Medição de pronta resposta da distribuição do tamanho de partícula aplicada ao processo de cristalização, Master Dissertation, 548.5(043) A161m. http://cassiopea.ipt.br/teses/2007_PI_Ricardo_Tadeu_Abrahao.pdf

Ahmed, M. Coloring of Plastics: Theory and Practice. New York: Van Nostrand Reinhold, 1979, p. 52.

Auer, G., Woditsch, P., Westerhaus, A., Kischkewitz, J; Griebler, O. D., and Liedekerke, M., 2009. Pigments, Inorganic, 2. White Pigments. In: Ullmann's Encyclopedia of Industrial Chemistry, p. 257 [online] doi: 10.1002/14356007.n20_n01,

Auger, J-C, Martinez, VA, Stout, B., 2009. Theoretical Study of the Scattering Efficiency of Rutile Titanium Dioxide Pigments as a Function of Their Spatial Dispersion. J. Coat. Technol. Res., 6(1) pp. 89-97.

Ayala, R. E., Hartley, P. A. and Parfitt, G. D., 1986. The Relevance of Powder/Liquid Wettability to the Cohesiveness of Carbon Black Agglomerates. Part. Part. Syst Charact, 3, pp. 26-31. doi: 10.1002/ppsc.19860030106

Baldyga et al., 2008. Deagglomeration Processes in high-shear devices. Chemical Engineering Research and Design, 86, pp. 1369-1381. http://www.sciencedirect.com/science/article/pii/S0263876208002542

Baneshi, M., Maruyama, S., Nakai, H. and Komiya, A., 2009. A new approach to optimizing pigmented coatings considering both thermal and aesthetic effects. Journal of Quantitative Spectroscopy and Radiative Transfer, 110(3), pp. 192-204. http://www.sciencedirect.com/science/article/pii/S0022407308002227

Bruehlman, J, Thomas, L. W., Gonick, E., 1961. Effect of particle size and pigment volume concentration on hiding power of titanium dioxide. Off. Dig. 33(433), pp. 252267.

Buxbaum G., Industrial Inorganic Pigments, Second Edition, John Wiley \& Sons, Germany, 1998, 302 pages

Buxbaum, G., 2007. Industrial Inorganic Pigments, 2nd edn. [online] pp. 43-82. DOI: $10.1002 / 9783527612116 . c h 2$

Chen, X., Gonsalves, K. E., Chow, G.-M., and Xiao, T. D., 1994. Homogeneous dispersion of nanostructured aluminum nitride in a polyimide matrix. Adv. Mater., 6, pp. 481-484. doi: 10.1002/adma.1994006060

Clarke, G., 1988. Title of Article Ind. Min. 251, pp. 17-31.

Constantinides, G. N. and Payatakes, A. C., 2000. Effects of precursor wetting films in immiscible displacement through porous media. Transport Porous Med., 38(3), pp. 291-317.

CRC Handbook of Chemistry and Physics, 92nd Edition, 2011-2012. 
Dang-Vu, T., Hupka, J., Drzymała, J., 2006. Impact of roughness on hydrophobicity of particles measured by the Washburn method. Physicochemical Problems of Mineral Processing, 40. http://www.ig.pwr.wroc.pl/minproc/journal/pdf/2006/s4552.pdf

De Backer, S. More Than a White Powder: Training Material for External Audiences. DuPont Titanium Technologies, Belgium, 2010.

Diebold, M. P., 2011. Title of Article. Journal of Coatings Technology and Research, 8(5), pp. 541-552.

Ding, Y. and Kawahara, M., 1999. Three-dimensional linear stability analysis of incompressible viscous flows using the finite element method. Int. J. Numer. Meth. Fluids, 31, pp. 451-479. doi: 10.1002/(SICl)1097-0363(19990930)31:2<451::AIDFLD885>3.0.CO;2-O

du Noüy, P. L., 1925. An Interfacial Tensiometer for Universal Use. The Journal of General Physiology 7(5), pp. 625-633. doi:10.1085/jgp.7.5.625.

Dunn, A. S. and Chong, L. C.-H., 1970. Application of the theory of colloid stability to the problem of particle formation in aqueous solutions of vinyl acetate. Brit. Poly. J., 2, pp. 49-59. doi: 10.1002/pi.4980020108

Evans, R., Davison, J. B., and Napper, D. H., 1972. Polymer Letter, 10, p. 449.

Farrokhpay, S., 2009. A review of polymeric dispersant stabilisation of titania pigment. Advances in Colloid and Interface Science, 151(1-2), pp. 24-32.

Hamid, Z. A., and Omar, A. M. A., 1999. The relation between anionic/non-ionic surfactant and electrodeposition of nickel-polytetrafluoro ethylene polymer composite. Anti-Corrosion Methods and Materials, 46(3), pp. 212-216.

Heertjes, P. M. and Kossen, N. W. F., 1967. Measuring the Contact Angles of Powder-Liquid Systems. Powder Technology, 1(1), pp. 33-42.

Hennart, S. L .A., Wildeboer, W. J., van Hee, P., and Meesters, G. M. H., 2010. Stability of Particle Suspensions after Fine Grinding. Powder Technology, 199(3), pp. 226-231.. (http://www.sciencedirect.com/science/article/pii/S003259101000029X)

Houivet, D., El Fallah, J. and Haussonne, J.-M., 2002. Dispersion and Grinding of Oxide Powders into an Aqueous Slurry. Journal of the American Ceramic Society, 85, pp. 321-328. doi: 10.1111/j.1151-2916.2002.tb00091.x

http://www.coleparmer.com/buy/product/45862-cannona-cannon-ubbelohdeviscometer-size-25-9721-k50.html [accessed 30 August 2012]

Josh, A., Rathi, S. and Deshpande, S., 2009. Analysis of the wetting behavior of pigments and the effectiveness of surfactants, coloration technology. Society of Dyes and Colourists. doi: 10.1111/j.1478-4408.2009.00180.x

Kiesvaara, J., and Yliruusib, J., 1993. The Use of the Washburn Method in Determining the Contact Angles of Lactose Powder. International Journal of Pharmaceutics, 92(1-3), pp. 81-88. 
König, P.-M., Roth, R., and Diet T.Y., 2006. Depletion Forces between Nonspherical Objects. Jour. American Physical Society, 74(4), SP - 041404, 2006/10/23/. http://link.aps.org/doi/10.1103/PhysRevE.74.041404.

Kosswig, K., 2000. Surfactants. Ullmann's Encyclopedia of Industrial Chemistry. Wiley-VCH Verlag GmbH \& Co. KGaA. http://dx.doi.org/10.1002/14356007.a25_747.

Law, S.-L., Lo, W.-Y., and Teh, G.-W., 1987. Effect of liposomes on suspension stability. J. Pharm. Sci., 76, pp. 545-547. doi: 10.1002/jps.2600760711

Mandzy, N, Grulke, E and Druffel, T, Breakage of TiO2 agglomerates in electrostatically stabilized aqueous dispersions. Powder Technology 160 (2005) 121 - 126, Elsevier.

Merkus, H. G., 2009. Dispersion of Powders in Air and in Liquids, Particle Size Measurements. Powder Technology Series, 17, pp. 117-136.

Mie, G., 1908. Beiträge zur Optik trüber Medien, speziell kolloidaler Metallösungen. Ann Phys, 25, pp. 377 - 445.

Millipore - https://www.bsilab.com/sites/default/files/Millipore_Elix.pdf (Accessed 20 September 2012)

Moorthaemer, B. and Sprakel, J., 2006. Improving the Stability of a Suspension. Pharmaceutical Technology Europe 18(2), pp. 30-34. [online] Available from: Business Source Corporate Plus, Ipswich, MA. [Accessed 10 August 2012]

Murphy, J., 2001. London, Modifying Specific Properties: Appearance-Black and White Pigmentation in Additives for Plastics Handbook, 2nd edn., page 73

Napper, D. H., 1968. Trans. Faraday Soc. 64, p. 1701.

Napper, D. H., 1970. J. Colloid Interface Sci. 32, p. 106.

Napper, D. H., 1977. Steric Stabilization. Journal of Colloid and Interface Science, 58(2), pp. 390-407.. (http://www.sciencedirect.com/science/article/pii/0021979777901503)

Napper, D. H., Kolloid, Z. Z., 1969. Polymer. Letter, 234, p. 1149.

Newitr, D. M. and Conway-Jones, J. M., 1958. A Contribution to the Theory and Practice of Granulation. Trans. Inst. Chem. Eng. 36, pp. 422-442.

Newman, A., Knipp, G. and Zografi, G., 2012. Assessing the performance of amorphous solid dispersions. J. Pharm. Sci., 10, pp. 1355-1377. doi: 10.1002/jps.23031

Oh, S.-Y., Cornie, J. A. and Russell, K. C., 2008. Particulate Wetting and Metal: Ceramic Interface Phenomena. 11th Annual Conference on Composites and Advanced Ceramic Materials: Ceramic Engineering and Science Proceedings, 8, ed. W. Smothers. Volume 8, Issue 7. Hoboken, NJ, USA: John Wiley \& Sons. doi: 10.1002/9780470320402.ch50 
Parfitt, G. D., 1981. Dispersion of Powders in Liquids with Special Reference to Pigments. London, UK: Applied Science Publishers,

Patton, T., 1979. Paint flow and pigment dispersion, a rheological approach to coating and ink technology, 2nd edn. Place of Publication: John Wiley \& Sons .

Pietsch, W., ed., 2008. Industrial Applications of Size Enlargement by Agglomeration. In: Agglomeration Set. Weinheim, Germany Wiley- $\mathrm{VCH}$ Verlag $\mathrm{GmbH}$. doi: 10.1002/9783527619788.ch6a

Pietsch, W., Hoffman, E., and Rumpf, H., 1969. Tensile Strength of Moist Agglomerates. American Chemical Society Product R\&D, 8(1), pp. 58-62. http://dx.doi.org/10.1021/i360029a009

Potente, H. and Kretschmer, K., 2002. Simulation and Evaluation of Compounding Processes. Macromol. Mater. Eng., 287, pp. 758-772. doi: $10.1002 /$ mame.200290005

Rohe, D., 1996. Chemische Industrie, 10, pp. 16-18.

Salvador, F., Sánchez-Jiménez, C., Sánchez-Montero, M. J., and Salvador, A., 2002. A review of the application of the BET equation to experimental data: the $C$ parameter in F. Rodriguez-Reinoso, B. McEnaney, J. Rouquerol and K. Unger, Eds. Studies in Surface Science and Catalysis, 144, pp. 379-386. (http://www.sciencedirect.com/science/article/pii/S0167299102801586).

Sato, K, Li, J, Kamiya, H and Ishigaki, T, Ultrasonic Dispersion of TiO2 Nanoparticles in Aqueous Suspension, J. Am. Ceram. Soc., 91 [8] 2481-2487 (2008), DOI: 10.1111/j.1551-2916.2008.02493.x, The American Ceramic Society

Schubert H., 1977. Tensile Strength and Capillary Pressure of Moist Agglomerates. In: K. V. S. Sastry (ed.). Agglomeration 77: Proc. 2nd Int. Symp. Agglomeration. Atlanta, GA: A.I.M.E. and Petroleum Eng. Inc., pp. 144-155.

Sing, K. S., Everett, D. H., Haul, R. A., Moscou, L., Pierotti, R. A., Rouquérol, J., et al., 1985. Reporting Physisorption Data for Gas/Solid Systems with Special Reference to the Determination of Surface Area and Porosity. Pure \& Applied Chemistry, 57, pp. 603-619.

Tavare, N., 1995. Industrial Crystallization, Process Simulation, Analysis and Design. New York: Plenum Press.

Thiele, E. S., and French, R. H., 2005. Light-scattering properties of representative, morphological rutile titania particles Studied Using a Finite-Element Method. Journal of the American Ceramic Society, vol. 81(3), pp. 469-479.

Tunstall, D. F., Hird, H. J., 1974. Effect of Particle Crowding on Scattering Power of TiO2 Pigments. J. Paint Technol., 46(588), pp. 33-40.

Vetec - ethanol - http://www.vetecquimica.com.br/home/detalhes/34640/produto (accessed 20 September 2012)

Vetec- n-hexane - http://www.vetecquimica.com.br/home/detalhes/34558/produto [accessed 20 September 2012] 
Vicent, M, Sánchez, E, Santacruz, I, and Morenoc, R, Dispersion of TiO2 nanopowders to obtain homogeneous nanostructured granules by spray-drying, Journal of the European Ceramic Society 31 (2011) 1413-1419. Elsevier.

Wang, Y, Zhang, J, Shen, X, Shi C, Wu J and Sunb, L, Dispersion investigation of TiO2 nanoparticles coated by pulsed RF plasma polymer, Materials Chemistry and Physics 98 (2006) 217-224, Elsevier.

Washburn, E. W., 1921. The Dynamics of Capillary Flow. Physical Review, 17(3), pp. 273-283. http://link.aps.org/doi/10.1103/PhysRev.17.273

Webb, P. 2003, Introduction to Chemical Adsorption Analytical Techniques and their Applications to Catalysis, Micromeritics Instrument Corp., MIC Technical Publications, Norcross, Georgia 30093

Williams, D., 1996. Eur. Chem. News, 17-23 June, pp. 36-39.

Wu, R.-Y., and Wei, W.-C. J., 2005. Deagglomeration Kinetics of Feedstocks with Granule Tetragonal Zirconia Polycrystalline Powder. Journal of the American Ceramic Society, 88: 1734-1739. doi: 10.1111/j.1551-2916.2005.00282.x

Wyckoff, R., 1965. Crystal Structure. New York: J. Wiley \& Sons.

Zhao, F.-Y. and Stewart, P. J., 2003. Deagglomeration of micronized benzodiazepines in dissolution media measured by laser diffraction particle sizing. Journal of Pharmacy and Pharmacology, 55, pp. 749-755. doi: $10.1211 / 002235703765951348$ 
Appendix 1

\begin{tabular}{|c|c|c|c|}
\hline $\begin{array}{l}\text { Liquid surface tension } \\
(\mathrm{mN} / \mathrm{m}) \text { and grade }\end{array}$ & $\begin{array}{c}\text { Adsorbed Volume } \\
(\mathrm{mL})\end{array}$ & $\begin{array}{l}\text { Adsorption } \\
\left.\text { Speed ( } \mathbf{g}^{2} / \mathbf{s}\right)\end{array}$ & $\begin{array}{l}\text { Adsorption Speed } \\
\left(\mathrm{mL}^{2} / \mathrm{s}\right)\end{array}$ \\
\hline \multicolumn{4}{|l|}{18} \\
\hline A & 3.29 & $4.68 \mathrm{E}-02$ & $1.09 \mathrm{E}-01$ \\
\hline$A^{\prime}$ & 3.22 & $5.08 \mathrm{E}-02$ & $1.18 \mathrm{E}-01$ \\
\hline$A^{\prime \prime}$ & 2.42 & 2.19E-02 & $5.11 \mathrm{E}-02$ \\
\hline B & 2.28 & $1.24 \mathrm{E}-02$ & $2.88 \mathrm{E}-02$ \\
\hline C & 2.07 & $8.24 \mathrm{E}-03$ & $1.92 \mathrm{E}-02$ \\
\hline \multicolumn{4}{|l|}{30} \\
\hline A & 0.12 & 4.06E-05 & $4.68 \mathrm{E}-05$ \\
\hline$A^{\prime}$ & 0.13 & 4.56E-05 & $5.26 \mathrm{E}-05$ \\
\hline A" & 1.06 & $3.28 \mathrm{E}-03$ & $3.78 \mathrm{E}-03$ \\
\hline B & 0.59 & $1.02 \mathrm{E}-03$ & $1.18 \mathrm{E}-03$ \\
\hline C & 0.44 & 5.57E-04 & $6.42 \mathrm{E}-04$ \\
\hline \multicolumn{4}{|l|}{46} \\
\hline A & 0.38 & 5.13E-04 & $5.13 \mathrm{E}-04$ \\
\hline$A^{\prime}$ & 0.34 & 3.77E-04 & 3.77E-04 \\
\hline$A^{\prime \prime}$ & 1.03 & $3.53 E-03$ & $3.53 \mathrm{E}-03$ \\
\hline B & 0.31 & $3.21 \mathrm{E}-04$ & $3.21 \mathrm{E}-04$ \\
\hline C & 0.45 & $6.85 \mathrm{E}-04$ & $6.85 \mathrm{E}-04$ \\
\hline \multicolumn{4}{|l|}{51} \\
\hline A & 0.32 & 3.40E-04 & $3.40 \mathrm{E}-04$ \\
\hline$A^{\prime}$ & 0.26 & $2.35 \mathrm{E}-04$ & $2.35 \mathrm{E}-04$ \\
\hline$A^{\prime \prime}$ & 1.28 & 5.47E-03 & 5.47E-03 \\
\hline B & 0.42 & $6.04 \mathrm{E}-04$ & $6.04 \mathrm{E}-04$ \\
\hline C & 0.39 & $5.15 \mathrm{E}-04$ & $5.15 \mathrm{E}-04$ \\
\hline \multicolumn{4}{|l|}{54} \\
\hline A & 0.13 & 5.93E-05 & $5.93 \mathrm{E}-05$ \\
\hline$A^{\prime}$ & 0.26 & $2.27 \mathrm{E}-04$ & $2.27 \mathrm{E}-04$ \\
\hline$A^{\prime \prime}$ & 1.44 & $6.93 E-03$ & $6.93 \mathrm{E}-03$ \\
\hline B & 0.41 & $5.54 \mathrm{E}-04$ & $5.54 \mathrm{E}-04$ \\
\hline C & 0.45 & $6.85 \mathrm{E}-04$ & $6.85 \mathrm{E}-04$ \\
\hline \multicolumn{4}{|l|}{59} \\
\hline A & 0.23 & $1.71 \mathrm{E}-04$ & $1.71 \mathrm{E}-04$ \\
\hline$A^{\prime}$ & 0.39 & $5.05 E-04$ & $5.05 \mathrm{E}-04$ \\
\hline$A^{\prime \prime}$ & 1.88 & $1.18 \mathrm{E}-02$ & $1.18 \mathrm{E}-02$ \\
\hline B & 0.43 & $6.10 \mathrm{E}-04$ & $6.10 \mathrm{E}-04$ \\
\hline C & 0.54 & $9.91 \mathrm{E}-04$ & $9.91 \mathrm{E}-04$ \\
\hline \multicolumn{4}{|l|}{73} \\
\hline A & 0.48 & $7.78 \mathrm{E}-04$ & $7.78 \mathrm{E}-04$ \\
\hline$A^{\prime}$ & 0.19 & $1.25 \mathrm{E}-04$ & $1.25 \mathrm{E}-04$ \\
\hline$A^{\prime \prime}$ & 1.24 & $5.15 \mathrm{E}-03$ & $5.15 \mathrm{E}-03$ \\
\hline B & 0.58 & $1.11 \mathrm{E}-03$ & $1.11 \mathrm{E}-03$ \\
\hline c & 0.53 & $9.37 \mathrm{E}-04$ & $9.37 \mathrm{E}-04$ \\
\hline
\end{tabular}

